TITLE:

\title{
Dynamics of partially localized brane systems
}

AUTHOR(S):

Minamitsuji, Masato; Uzawa, Kunihito

CITATION:

Minamitsuji, Masato ...[et al]. Dynamics of partially localized brane systems. Physical Review D 2011, 84(12)

ISSUE DATE:

2011-12

URL:

http://hdl.handle.net/2433/154636

RIGHT:

(C) 2011 American Physical Society 
PHYSICAL REVIEW D 84, 126006 (2011)

\title{
Dynamics of partially localized brane systems
}

\author{
Masato Minamitsuji and Kunihito Uzawa \\ Yukawa Institute for Theoretical Physics, Kyoto University, Kyoto 606-8502, Japan \\ Department of Physics, Kinki University, Higashi-Osaka, Osaka 577-8502, Japan
}

(Received 7 September 2011; published 9 December 2011)

\begin{abstract}
We study dynamical partially-localized brane solutions in higher dimensions. We give new descriptions of the relevant solutions of dynamical branes which are localized along both the overall and relative transverse directions. The starting point is a system of $p_{r}$-branes ending on a $p_{s}$-brane with a timedependent warp factor. This system can be related to $\mathrm{D} p_{r}-\mathrm{Dp}_{s}$ brane system in string theory, where one brane is localized at the delocalized other brane. We then show that these give Friedmann-LemaitreRobertson-Walker cosmological solutions. Our approach leads to a new and manifest description of the brane configurations near the delocalized branes, and new solutions in the wave or KK-monopole background in terms of certain partial differential equations in $D$ dimensions including ten and eleven dimensions.
\end{abstract}

DOI: 10.1103/PhysRevD.84.126006

PACS numbers: 11.25. $-\mathrm{w}, 11.27 .+\mathrm{d}, 98.80 . \mathrm{Cq}$

\section{INTRODUCTION}

In recent years much effort has been devoted to the construction of cosmological models in string theory produced by enlarging static $p$-brane solutions [1-5]. Although these calculations are complicated by the occurrence of time-dependences, there has been active development in constructing time-dependent supergravity solutions of $p$-branes and other solitons in string theory [6-13]. These classical solutions in string theory made it possible to discuss the dynamics such as cosmological evolution of our Universe and brane collision within the framework of string theory. In these studies, brane world models were obtained by wrapping or intersecting higherdimensional $p$-branes around compact manifolds. In the course of compactifying $p$-branes, the dynamical solutions become smeared or delocalized along the compactified directions, which include possibly some of overall transverse directions and relative transverse directions that corresponds to the transverse directions which are longitudinal to some of other constituent branes. Such intersecting $p$-brane solutions in higher dimensions thus become localized only along the relative or overall transverse directions. The dynamical intersecting brane solutions which we have mainly constructed are such delocalized type $[1,2,4,5]$. There are several works to construct the static localized intersecting brane solutions with the restricted ansatz of fields which has the same form as the corresponding delocalized intersecting brane solutions [14-17]. The equations of motion along with such simplified assumption for fields require that one of the branes has to be delocalized on the relative transverse directions. However, it is difficult to obtain the exact localized solutions even if we use such simplified ansatz because harmonic functions that specify branes satisfy coupled partial differential equations. The solutions of these differential equations in general have a complicated form. On the other hand, the dynamical localized intersecting brane solution is not well-known, and nobody mentions the explicit expressions for harmonic functions. This article will describe a method of dealing with the extension of the time-dependent solutions in the partially localized intersecting brane system, where branes are localized along the relative transverse directions but delocalized along the overall transverse directions [18]. For the purposes of construction of cosmological model, we employ the same ansatz of fields as the static $p$-brane solutions. It is, in general, possible to derive intersecting brane solutions in terms of applying duality transformations in string theory. For instance, we compactify the direction which becomes delocalized through smearing or uniform array of branes along it to apply T-duality transformations in the transverse directions. Then, the power of the radial coordinate in the harmonic function changes. Hence, we will construct such localized intersecting brane solutions case by case.

It is the purpose of this paper to construct various explicit partially localized intersecting dynamical brane solutions in various dimensions. We give classification of these dynamical intersecting brane solutions involving two branes, and discuss the application of these solutions to cosmology. We also study the arbitrary single brane on the KK-monopole and wave background. It is possible to derive the time-dependent solution if the form of the static solution is explicitly known, so calculations have generally relied on an assumption of fields and even strictly metric form. Also, even where a coupling between scalar field and gauge field strength in the action is known, the intersection rule of the brane can be obtained explicitly. Since a warp factor arises from a field strength, the dynamics of a system composed of two branes can be characterized by two warp factors arising from two field strengths. For M-branes and D-branes, among these warp factors only one function can depend on time. 
The procedure is described here in generally higherdimensional gravity model as well as the supergravity, the solutions of a D-brane or M-brane in a wave or KKmonopole background. This is simple enough to illustrate the use of the method without the general idea being lost in the complications of higher-dimensional gravity theory, and yet sufficiently general so that we can see how to deal with an arbitrary expansion. As we will see, these methods yield a prescription for intersecting brane solutions that depend not only on the overall transverse directions, but world-volume and the relative transverse directions.

The paper is organized as follows. In Sec. II, we show that the partially localized dynamical intersecting brane solutions of two $p$-branes exist as an almost immediate generalization of the static brane solution where one of branes is delocalized. We will also study explicit partially localized $p$-brane solutions in KK-monopole or wave background. We will apply these solutions to construct various explicit partially localized intersecting M-brane solutions in Sec. III, and various partially localized intersecting brane solutions in ten dimensions in Sec. IV. We then go on in Sec. V to apply these solutions to cosmology. Section VI is devoted to discussions.

\section{THE INTERSECTION OF TWO BRANES IN $D$-DIMENSIONAL THEORY}

We study the dynamical brane in $D$-dimensional theory. We describe the relation of the partially localized static brane solution to time-dependent solutions in $D$-dimensions. We also study solutions in the wave and KK-monopole background.

\section{A. The intersection of two $p$-branes in $D$-dimensional theory}

In this section, we consider a $D$-dimensional theory composed of the metric $g_{M N}$, dilaton $\phi$, and two antisymmetric tensor fields of rank $\left(p_{r}+2\right)$ and $\left(p_{s}+2\right)$ :

$$
\begin{aligned}
S= & \frac{1}{2 \kappa^{2}} \int\left[R * \mathbf{1}-\frac{1}{2} d \phi \wedge * d \phi\right. \\
& -\frac{1}{2} \frac{1}{\left(p_{r}+2\right) !} \mathrm{e}^{\epsilon_{r} c_{r} \phi} F_{\left(p_{r}+2\right)} \wedge * F_{\left(p_{r}+2\right)} \\
& \left.-\frac{1}{2} \frac{1}{\left(p_{s}+2\right) !} \mathrm{e}^{\epsilon_{s} c_{s} \phi} F_{\left(p_{s}+2\right)} \wedge * F_{\left(p_{s}+2\right)}\right],
\end{aligned}
$$

where $\kappa^{2}$ is the $D$-dimensional gravitational constant, $*$ is the Hodge operator in the $D$-dimensional space-time, $F_{\left(p_{r}+2\right)}$ and $F_{\left(p_{s}+2\right)}$ are $\left(p_{r}+2\right)$-form, $\left(p_{s}+2\right)$-form field strengths, respectively. And $c_{I}, \epsilon_{I}(I=r, s)$ are constants given by

$$
\begin{aligned}
c_{I}^{2} & =N_{I}-\frac{2\left(p_{I}+1\right)\left(D-p_{I}-3\right)}{D-2}, \\
\epsilon_{I} & = \begin{cases}+ & \text { if } p_{I}-\text { brane is electric } \\
- & \text { if } p_{I}-\text { brane is magnetic }\end{cases}
\end{aligned}
$$

Here $N_{I}$ is constant. The field strength $F_{\left(p_{r}+2\right)}, F_{\left(p_{s}+2\right)}$ are given by the $\left(p_{r}+1\right)$-form, $\left(p_{s}+1\right)$-form gauge potentials $A_{\left(p_{r}+1\right)}, A_{\left(p_{s}+1\right)}$, respectively

$$
F_{\left(p_{r}+2\right)}=d A_{\left(p_{r}+1\right)}, \quad F_{\left(p_{s}+2\right)}=d A_{\left(p_{s}+1\right)} .
$$

After varying the action with respect to the metric, the dilaton, and the $\left(p_{r}+1\right)$-form and $\left(p_{s}+1\right)$-form gauge fields, we obtain the field equations,

$$
\begin{aligned}
& R_{M N}= \frac{1}{2} \partial_{M} \phi \partial_{N} \phi+\frac{1}{2} \frac{\mathrm{e}^{\epsilon_{r} c_{r} \phi}}{\left(p_{r}+2\right) !}\left[\left(p_{r}+2\right) F_{M A_{2} \cdots A_{\left(p_{r}+2\right)}} F_{N}^{A_{2} \cdots A_{\left(p_{r}+2\right)}}-\frac{p_{r}+1}{D-2} g_{M N} F_{\left(p_{r}+2\right)}^{2}\right] \\
&+\frac{1}{2} \frac{\mathrm{e}^{\epsilon_{s} c_{s} \phi}}{\left(p_{s}+2\right) !}\left[\left(p_{s}+2\right) F_{M A_{2} \cdots A_{\left(p_{s}+2\right)}} F_{N}^{A_{2} \cdots A_{\left(p_{s}+2\right)}}-\frac{p_{s}+1}{D-2} g_{M N} F_{\left(p_{s}+2\right)}^{2}\right], \\
& d * d \phi-\frac{1}{2} \frac{\epsilon_{r} c_{r}}{\left(p_{r}+2\right) !} \mathrm{e}^{\epsilon_{r} c_{r} \phi} F_{\left(p_{r}+2\right)} \wedge * F_{\left(p_{r}+2\right)}-\frac{1}{2} \frac{\epsilon_{s} c_{s}}{\left(p_{s}+2\right) !} \mathrm{e}^{\epsilon_{s} c_{s} \phi} F_{\left(p_{s}+2\right)} \wedge * F_{\left(p_{s}+2\right)}=0, \\
& d\left[\mathrm{e}^{\epsilon_{r} c_{r} \phi} * F_{\left(p_{r}+2\right)}\right]=0, \\
& d\left[\mathrm{e}^{\epsilon_{s} c_{s} \phi} * F_{\left(p_{s}+2\right)}\right]=0 .
\end{aligned}
$$

To solve the field equations, we assume that the $D$-dimensional metric takes the form

$$
\begin{aligned}
d s^{2}= & h_{r}^{a_{r}}(x, y, z) h_{s}^{a_{s}}(x, v, z) q_{\mu \nu}(\mathrm{X}) d x^{\mu} d x^{\nu} \\
& +h_{r}^{b_{r}}(x, y, z) h_{s}^{a_{s}}(x, v, z) \gamma_{i j}\left(\mathrm{Y}_{1}\right) d y^{i} d y^{j} \\
& +h_{r}^{a_{r}}(x, y, z) h_{s}^{b_{s}}(x, v, z) w_{m n}\left(\mathrm{Y}_{2}\right) d v^{m} d v^{n} \\
& +h_{r}^{b_{r}}(x, y, z) h_{s}^{b_{s}}(x, v, z) u_{a b}(\mathrm{Z}) d z^{a} d z^{b},
\end{aligned}
$$

where $q_{\mu \nu}$ is the $(p+1)$-dimensional metric which depends only on the $(p+1)$-dimensional coordinates $x^{\mu}, \gamma_{i j}$ is the $\left(p_{s}-p\right)$-dimensional metric which depends only on the $\left(p_{s}-p\right)$-dimensional coordinates $y^{i}, w_{m n}$ is the $\left(p_{r}-p\right)$-dimensional metric which depends only on the $\left(p_{r}-p\right)$-dimensional coordinates $v^{m}$ and finally $u_{a b}$ is the $\left(D+p-p_{r}-p_{s}-1\right)$-dimensional metric which depends only on the $\left(D+p-p_{r}-p_{s}-1\right)$-dimensional coordinates $z^{a}$. The parameters $a_{I}(I=r, s)$ and $b_{I}(I=$ $r, s)$ in the metric (5) are given by 
DYNAMICS OF PARTIALLY LOCALIZED BRANE SYSTEMS

PHYSICAL REVIEW D 84, 126006 (2011)

$$
a_{I}=-\frac{4\left(D-p_{I}-3\right)}{N_{I}(D-2)}, \quad b_{I}=\frac{4\left(p_{I}+1\right)}{N_{I}(D-2)} .
$$

The brane configuration is given as follows:

The $D$-dimensional metric (5) implies that the solutions are characterized by two functions, $h_{r}$ and $h_{s}$, which depend on the coordinates transverse to the brane as well as the world-volume coordinate (see Table I). For the configurations of two branes, the powers of harmonic functions have to obey the intersection rule, and then split the coordinates in three parts. One is the overall worldvolume coordinates, $\{x\}$, which are common to the two branes. The others are overall transverse coordinates, $\{z\}$, and the relative transverse coordinates, $\{y\}$ and $\{v\}$, which are transverse to only one of the two branes. Each of $h_{r}$ and $h_{s}$ depends not only on overall transverse coordinates but also on the corresponding relative coordinates: $h_{r}=$ $h_{r}(x, y, z), h_{s}=h_{s}(x, v, z)$.

We also assume that the scalar field $\phi$ and the gauge field strengths $F_{\left(p_{r}+2\right)}, F_{\left(p_{s}+2\right)}$ are given by

$$
\begin{aligned}
\mathrm{e}^{\phi} & =h_{r}^{2 \epsilon_{r} c_{r} / N_{r}} h_{s}^{2 \epsilon_{s} c_{s} / N_{s}}, \\
F_{\left(p_{r}+2\right)} & =\frac{2}{\sqrt{N_{r}}} d\left[h_{r}^{-1}(x, y, z)\right] \wedge \Omega(\mathrm{X}) \wedge \Omega\left(\mathrm{Y}_{2}\right), \\
F_{\left(p_{s}+2\right)} & =\frac{2}{\sqrt{N_{s}}} d\left[h_{s}^{-1}(x, v, z)\right] \wedge \Omega(\mathrm{X}) \wedge \Omega\left(\mathrm{Y}_{1}\right),
\end{aligned}
$$

where $\Omega(\mathrm{X}), \Omega\left(\mathrm{Y}_{1}\right)$, and $\Omega\left(\mathrm{Y}_{2}\right)$ denote the volume $(p+1)$-form, $\left(p_{s}-p\right)$-form, and $\left(p_{r}-p\right)$-form respectively

$$
\begin{aligned}
\Omega(\mathrm{X}) & =\sqrt{-q} d x^{0} \wedge d x^{1} \wedge \cdots \wedge d x^{p} \\
\Omega\left(\mathrm{Y}_{1}\right) & =\sqrt{\gamma} d y^{1} \wedge d y^{2} \wedge \cdots \wedge d y^{p_{s}-p} \\
\Omega\left(\mathrm{Y}_{2}\right) & =\sqrt{w} d v^{1} \wedge d v^{2} \wedge \cdots \wedge d v^{p_{r}-p}
\end{aligned}
$$

Here, $q, \gamma$, and $w$ are the determinants of the metrics $q_{\mu \nu}$, $\gamma_{i j}$, and $w_{m n}$, respectively.

First we consider the Einstein Eq. (4a). Using the assumptions (5) and (7), the Einstein equations are given by

$$
\begin{aligned}
& R_{\mu \nu}(\mathrm{X})-\frac{4}{N_{r}} h_{r}^{-1} D_{\mu} D_{\nu} h_{r}-\frac{4}{N_{s}} h_{s}^{-1} D_{\mu} D_{\nu} h_{s}+\frac{2}{N_{r}} \partial_{\mu} \ln h_{r}\left[\left(1-\frac{4}{N_{r}}\right) \partial_{\nu} \ln h_{r}-\frac{4}{N_{s}} \partial_{\nu} \ln h_{s}\right] \\
& +\frac{2}{N_{s}} \partial_{\mu} \ln h_{s}\left[\left(1-\frac{4}{N_{s}}\right) \partial_{\nu} \ln h_{s}-\frac{4}{N_{r}} \partial_{\nu} \ln h_{r}\right]-\frac{1}{2} q_{\mu \nu} h_{r}^{-4 / N_{r}} h_{s}^{-4 / N_{s}}\left[a_{r} h_{r}^{-1}\left(h_{s}^{4 / N_{s}} \triangle_{\mathrm{Y}_{1}} h_{r}+\triangle_{\mathrm{Z}} h_{r}\right)\right. \\
& \left.+a_{s} h_{s}^{-1}\left(h_{r}^{4 / N_{r}} \triangle_{\mathrm{Y}_{2}} h_{s}+\triangle_{\mathrm{Z}} h_{s}\right)\right]-\frac{1}{2} q_{\mu \nu}\left[a_{r} h_{r}^{-1} \triangle_{\mathrm{X}} h_{r}-a_{r} q^{\rho \sigma} \partial_{\rho} \ln h_{r}\left\{\left(1-\frac{4}{N_{r}}\right) \partial_{\sigma} \ln h_{r}-\frac{4}{N_{s}} \partial_{\sigma} \ln h_{s}\right\}\right. \\
& \left.+a_{s} h_{s}^{-1} \triangle_{\mathrm{X}} h_{s}-a_{s} q^{\rho \sigma} \partial_{\rho} \ln h_{s}\left\{\left(1-\frac{4}{N_{s}}\right) \partial_{\sigma} \ln h_{s}-\frac{4}{N_{r}} \partial_{\sigma} \ln h_{r}\right\}\right]=0, \\
& \frac{2}{N_{r}} h_{r}^{-1}\left(\partial_{\mu} \partial_{i} h_{r}+\frac{4}{N_{s}} \partial_{\mu} \ln h_{s} \partial_{i} h_{r}\right)=0 \\
& \frac{2}{N_{s}} h_{s}^{-1}\left(\partial_{\mu} \partial_{m} h_{s}+\frac{4}{N_{r}} \partial_{\mu} \ln h_{r} \partial_{m} h_{s}\right)=0, \frac{2}{N_{r}} h_{r}^{-1} \partial_{\mu} \partial_{a} h_{r}+\frac{2}{N_{s}} h_{s}^{-1} \partial_{\mu} \partial_{a} h_{s}=0, \\
& R_{i j}\left(\mathrm{Y}_{1}\right)-\frac{1}{2} h_{r}^{4 / N_{r}} \gamma_{i j}\left[b_{r} h_{r}^{-1} \triangle_{\mathrm{X}} h_{r}-b_{r} q^{\rho \sigma} \partial_{\rho} \ln h_{r}\left\{\left(1-\frac{4}{N_{r}}\right) \partial_{\sigma} \ln h_{r}-\frac{4}{N_{s}} \partial_{\sigma} \ln h_{s}\right\}+a_{s} h_{s}^{-1} \triangle_{\mathrm{X}} h_{s}\right. \\
& \left.-a_{s} q^{\rho \sigma} \partial_{\rho} \ln h_{s}\left\{\left(1-\frac{4}{N_{s}}\right) \partial_{\sigma} \ln h_{s}-\frac{4}{N_{r}} \partial_{\sigma} \ln h_{r}\right\}\right]-\frac{1}{2} \gamma_{i j} h_{s}^{-4 / N_{s}}\left(b_{r} h_{r}^{-1} \triangle_{\mathrm{Z}} h_{r}+a_{s} h_{s}^{-1} \triangle_{\mathrm{Z}} h_{s}\right)=0 \text {, } \\
& \frac{8}{N_{r} N_{s}(D-2)^{2}}\left[\left(p_{r}+1\right)\left(p_{s}+1\right)-(D-2)\left(p_{r}-p_{s}\right)\right] \partial_{i} \ln h_{r} \partial_{m} \ln h_{s}=0 \\
& R_{m n}\left(\mathrm{Y}_{2}\right)-\frac{1}{2} h_{s}^{4 / N_{s}} w_{m n}\left[a_{r} h_{r}^{-1} \triangle_{\mathrm{X}} h_{r}-a_{r} q^{\rho \sigma} \partial_{\rho} \ln h_{r}\left\{\left(1-\frac{4}{N_{r}}\right) \partial_{\sigma} \ln h_{r}-\frac{4}{N_{s}} \partial_{\sigma} \ln h_{s}\right\}+b_{s} h_{s}^{-1} \triangle_{\mathrm{X}} h_{s}\right. \\
& \left.-b_{s} q^{\rho \sigma} \partial_{\rho} \ln h_{s}\left\{\left(1-\frac{4}{N_{s}}\right) \partial_{\sigma} \ln h_{s}-\frac{4}{N_{r}} \partial_{\sigma} \ln h_{r}\right\}\right]-\frac{1}{2} w_{m n} h_{r}^{-4 / N_{r}}\left(a_{r} h_{r}^{-1} \triangle_{\mathrm{Z}} h_{r}+b_{s} h_{s}^{-1} \triangle_{\mathrm{Z}} h_{s}\right)=0 \text {, } \\
& R_{a b}(\mathrm{Z})-\frac{1}{2} h_{r}^{4 / N_{r}} h_{s}^{4 / N_{s}} u_{a b}\left[b_{r} h_{r}^{-1} \triangle_{\mathrm{X}} h_{r}-b_{r} q^{\rho \sigma} \partial_{\rho} \ln h_{r}\left\{\left(1-\frac{4}{N_{r}}\right) \partial_{\sigma} \ln h_{r}-\frac{4}{N_{s}} \partial_{\sigma} \ln h_{s}\right\}+b_{s} h_{s}^{-1} \triangle_{\mathrm{X}} h_{s}\right. \\
& \left.-b_{s} q^{\rho \sigma} \partial_{\rho} \ln h_{s}\left\{\left(1-\frac{4}{N_{s}}\right) \partial_{\sigma} \ln h_{s}-\frac{4}{N_{r}} \partial_{\sigma} \ln h_{r}\right\}\right]-\frac{1}{2} u_{a b}\left(b_{r} h_{r}^{-1} \triangle_{\mathrm{Z}} h_{r}+b_{s} h_{s}^{-1} \triangle_{\mathrm{Z}} h_{s}\right)=0 \text {, }
\end{aligned}
$$

where $D_{\mu}$ is the covariant derivative with respect to the metric $q_{\mu \nu}$, and $\triangle_{\mathrm{X}}, \triangle_{\mathrm{Y}_{1}}, \triangle_{\mathrm{Y}_{2}}, \triangle_{\mathrm{Z}}$ the Laplace operators on $\mathrm{X}$, $\mathrm{Y}_{2}$, $\mathrm{Y}_{1}, \mathrm{Z}$ spaces, and $R_{\mu \nu}(\mathrm{X}), R_{i j}\left(\mathrm{Y}_{1}\right), R_{m n}\left(\mathrm{Y}_{2}\right)$, and $R_{a b}(\mathrm{Z})$ are the Ricci tensors associated with the metrics $q_{\mu \nu}(\mathrm{X}), \gamma_{i j}\left(\mathrm{Y}_{1}\right)$, $w_{m n}\left(\mathrm{Y}_{2}\right)$ and $u_{a b}(\mathrm{Z})$, respectively, and $\chi$ is defined by 
TABLE I. Intersections of two $p$-branes in the metric (5).

\begin{tabular}{|c|c|c|c|c|c|c|c|c|c|c|c|c|c|c|}
\hline Case & & 0 & 1 & $\cdots$ & $p$ & $p+1$ & $\cdots$ & $p_{s}$ & $p_{s}+1$ & $\cdots$ & $p_{s}+p_{r}-p$ & $p_{s}+p_{r}-p+1$ & $\cdots$ & $D-1$ \\
\hline & $p_{r}$ & $\circ$ & $\circ$ & $\circ$ & $\circ$ & & & & $\circ$ & $\circ$ & $\circ$ & & & \\
\hline \multirow[t]{2}{*}{$p_{r}-p_{s}$} & $p_{s}$ & $\circ$ & $\circ$ & $\circ$ & $\circ$ & $\circ$ & $\circ$ & $\circ$ & & & & & & \\
\hline & $x^{N}$ & $t$ & $x^{1}$ & $\cdots$ & $x^{p}$ & $y^{1}$ & $\cdots$ & $y^{p_{s}-p-1}$ & $v^{1}$ & $\cdots$ & $v^{p_{r}-p-1}$ & $z^{1}$ & $\cdots$ & $z^{D+p-p_{r}-p_{s}-1}$ \\
\hline
\end{tabular}

$$
\chi=p+1-\frac{\left(p_{r}+1\right)\left(p_{s}+1\right)}{D-2}+\frac{1}{2} \epsilon_{r} \epsilon_{s} c_{r} c_{s} .
$$

The relation $\chi=0$ is consistent with the intersection rule [1,2,4,5,19-22].

We see from Eqs. (9b)-(9d), that the warp factors $h_{r}$ and $h_{s}$ must take the form

$$
\begin{array}{ll}
h_{r}=h_{0}(x)+h_{1}(y, z), \quad h_{s}=h_{s}(v, z), & \text { for } \partial_{\mu} h_{s}=0, \\
h_{r}=h_{r}(y, z), \quad h_{s}=k_{0}(x)+k_{1}(v, z), & \text { for } \partial_{\mu} h_{r}=0 .
\end{array}
$$

Let us first consider the case $\partial_{\mu} h_{s}=0$. The components of the Einstein Eqs. (9) are rewritten as

$$
\begin{aligned}
& R_{\mu \nu}(\mathrm{X})-\frac{2}{N_{r}}\left[h_{r}^{-1} D_{\mu} D_{\nu} h_{0}-2\left(1-\frac{4}{N_{r}}\right) \partial_{\mu} \ln h_{r} \partial_{\nu} \ln h_{r}\right]-\frac{1}{2} q_{\mu \nu} h_{r}^{-4 / N_{r}} h_{s}^{-4 / N_{s}}\left[a_{r} h_{r}^{-1}\left(h_{s}^{4 / N_{s}} \triangle_{\mathrm{Y}_{1}} h_{1}+\triangle_{\mathrm{Z}} h_{1}\right)\right. \\
& \left.\quad+a_{s} h_{s}^{-1}\left(h_{r}^{4 / N_{r}} \triangle_{\mathrm{Y}_{2}} h_{s}+\triangle_{\mathrm{Z}} h_{s}\right)\right]-\frac{1}{2} a_{r} q_{\mu \nu}\left[h_{r}^{-1} \triangle_{\mathrm{X}} h_{0}-\left(1-\frac{4}{N_{r}}\right) q^{\rho \sigma} \partial_{\rho} \ln h_{r} \partial_{\sigma} \ln h_{r}\right]=0 \\
& R_{i j}\left(\mathrm{Y}_{1}\right)-\frac{1}{2} b_{r} h_{r}^{4 / N_{r}} \gamma_{i j}\left[h_{r}^{-1} \triangle_{\mathrm{X}} h_{0}-\left(1-\frac{4}{N_{r}}\right) q^{\rho \sigma} \partial_{\rho} \ln h_{r} \partial_{\sigma} \ln h_{r}\right]-\frac{1}{2} \gamma_{i j} h_{s}^{-4 / N_{s}}\left[b_{r} h_{r}^{-1}\left(h_{s}^{4 / N_{s}} \triangle_{\mathrm{Y}_{1}} h_{1}+\triangle_{\mathrm{Z}} h_{1}\right)\right. \\
& \left.\quad+a_{s} h_{s}^{-1}\left(h_{r}^{4 / N_{r}} \triangle_{\mathrm{Y}_{2}} h_{s}+\triangle_{\mathrm{Z}} h_{s}\right)\right]=0, \\
& \quad \frac{8}{N_{r} N_{s}(D-2)^{2}}\left[\left(p_{r}+1\right)\left(p_{s}+1\right)-(D-2)\left(p_{r}-p_{s}\right)\right] \partial_{i} \ln h_{r} \partial_{m} \ln h_{s}=0, \\
& R_{m n}\left(\mathrm{Y}_{2}\right)-\frac{1}{2} a_{r} h_{s}^{4 / N_{s}} w_{m n}\left[h_{r}^{-1} \triangle_{\mathrm{X}} h_{0}-\left(1-\frac{4}{N_{r}}\right) q^{\rho \sigma} \partial_{\rho} \ln h_{r} \partial_{\sigma} \ln h_{r}\right]-\frac{1}{2} w_{m n} h_{r}^{-4 / N_{r}}\left[a_{r} h_{r}^{-1}\left(h_{s}^{4 / N_{s}} \triangle_{\mathrm{Y}_{1}} h_{1}+\triangle_{\mathrm{Z}} h_{1}\right)\right. \\
& \left.\quad+b_{s} h_{s}^{-1}\left(h_{r}^{4 / N_{r}} \triangle_{\mathrm{Y}_{2}} h_{s}+\triangle_{\mathrm{Z}} h_{s}\right)\right]=0, \\
& R_{a b}(\mathrm{Z})-\frac{1}{2} b_{r} h_{r}^{4 / N_{r}} h_{s}^{4 / N_{s}} u_{a b}\left[h_{r}^{-1} \triangle_{\mathrm{X}} h_{0}-\left(1-\frac{4}{N_{r}}\right) q^{\rho \sigma} \partial_{\rho} \ln h_{r} \partial_{\sigma} \ln h_{r}\right]-\frac{1}{2} u_{a b}\left[b_{r} h_{r}^{-1}\left(h_{s}^{4 / N_{s}} \triangle_{\mathrm{Y}_{1}} h_{1}+\triangle_{\mathrm{Z}} h_{1}\right)\right. \\
& \left.\quad+b_{s} h_{s}^{-1}\left(h_{r}^{4 / N_{r}} \triangle_{\mathrm{Y}_{2}} h_{s}+\triangle_{\mathrm{Z}} h_{s}\right)\right]=0 .
\end{aligned}
$$

Let us next consider the gauge field Eqs. (4c) and (4d). Under the assumption (7b) and (7c), we find

$$
\begin{aligned}
& d\left[h_{s}^{4(\chi+1) / N_{s}} \partial_{i} h_{r}\left(*_{\mathrm{Y}_{1}} d y^{i}\right) \wedge \Omega(\mathrm{Z})\right. \\
& \left.\quad+h_{s}^{4 \chi / N_{s}} \partial_{a} h_{r}\left(*_{\mathrm{Z}} d z^{a}\right) \wedge \Omega\left(\mathrm{Y}_{1}\right)\right]=0, \\
& d\left[h_{r}^{4(\chi+1) / N_{r}} \partial_{m} h_{s}\left(*_{\mathrm{Y}_{2}} d v^{m}\right) \wedge \Omega(\mathrm{Z})\right. \\
& \left.\quad+h_{r}^{4 \chi / N_{r}} \partial_{a} h_{s}\left(*_{\mathrm{Z}} d z^{a}\right) \wedge \Omega\left(\mathrm{Y}_{2}\right)\right]=0,
\end{aligned}
$$

where $*_{\mathrm{Y}_{1}}, *_{\mathrm{Y}_{2}}$, and $*_{\mathrm{Z}}$ denote the Hodge operator on $\mathrm{Y}_{1}$, $\mathrm{Y}_{2}$, and $\mathrm{Z}$, respectively, and $\chi$ is defined by (10). Then, for $\chi=0$, the Eq. (13a) leads to

$$
\begin{aligned}
h_{s} \triangle_{\mathrm{Y}_{1}} h_{r}+\triangle_{\mathrm{Z}} h_{r} & =0, \\
\partial_{\mu} \partial_{i} h_{r}+\frac{4}{N_{s}} \partial_{\mu} \ln h_{s} \partial_{i} h_{r} & =0, \\
\partial_{\mu} \partial_{a} h_{r} & =0,
\end{aligned}
$$

where $\triangle_{Y_{1}}$, and $\triangle_{Z}$ are the Laplace operators on the space of $\mathrm{Y}_{1}$, and $\mathrm{Z}$, respectively. On the other hand, it follows from (13b) that

$$
\begin{aligned}
h_{r} \triangle_{\mathrm{Y}_{2}} h_{s}+\triangle_{\mathrm{Z}} h_{s} & =0, \\
\partial_{\mu} \partial_{m} h_{s}+\frac{4}{N_{r}} \partial_{\mu} \ln h_{r} \partial_{m} h_{s} & =0, \\
\partial_{\mu} \partial_{a} h_{s} & =0,
\end{aligned}
$$

where $\triangle_{Y_{2}}$ is the Laplace operator on the space of $Y_{2}$.

Finally we should consider the scalar field equation. Substituting Eqs. (7) and (11) and the intersection rule $\chi=0$ into Eq. (4b), we obtain

$$
\begin{aligned}
& \frac{\epsilon_{r} c_{r}}{N_{r}} h_{r}^{4 / N_{r}} h_{s}^{4 / N_{s}}\left[h_{r}^{-1} \triangle_{\mathrm{X}} h_{0}-\left(1-\frac{4}{N_{r}}\right) q^{\rho \sigma} \partial_{\rho} \ln h_{r} \partial_{\sigma} \ln h_{r}\right] \\
& \quad+\frac{\epsilon_{r} c_{r}}{N_{r}} h_{r}^{-1}\left(h_{s}^{4 / N_{s}} \triangle_{\mathrm{Y}_{1}} h_{1}+\triangle_{\mathrm{Z}} h_{1}\right) \\
& \quad+\frac{\epsilon_{s} c_{s}}{N_{s}} h_{s}^{-1}\left(h_{r}^{4 / N_{r}} \triangle_{\mathrm{Y}_{2}} h_{s}+\triangle_{\mathrm{Z}} h_{s}\right)=0
\end{aligned}
$$


Thus, the warp factors $h_{r}$ and $h_{s}$ should satisfy the equations

$$
\begin{gathered}
h_{r} \triangle_{\mathrm{X}} h_{0}+\left(1-\frac{4}{N_{r}}\right) q^{\rho \sigma} \partial_{\rho} h_{0} \partial_{\sigma} h_{0}=0, \quad h_{s}^{4 / N_{s}} \triangle_{\mathrm{Y}_{1}} h_{1}+\triangle_{\mathrm{Z}} h_{1}=0, \\
h_{r}^{4 / N_{r}} \triangle_{\mathrm{Y}_{2}} h_{s}+\triangle_{\mathrm{Z}} h_{s}=0 .
\end{gathered}
$$

Combining these, we find that these field equations lead to

$$
\begin{aligned}
& R_{\mu \nu}(\mathrm{X})=0, \quad R_{i j}\left(\mathrm{Y}_{1}\right)=0, \quad R_{m n}\left(\mathrm{Y}_{2}\right)=0, \quad R_{a b}(\mathrm{Z})=0, \\
& h_{r}=h_{0}(x)+h_{1}(y, z), \quad h_{s}=h_{s}(v, z), \quad \partial_{i} h_{r} \partial_{m} h_{s}=0, \\
& D_{\mu} D_{\nu} h_{0}=0, \quad\left(1-\frac{4}{N_{r}}\right) \partial_{\mu} h_{0} \partial_{\nu} h_{0}=0, \quad h_{s}^{4 / N_{s}} \triangle_{\mathrm{Y}_{1}} h_{1}+\triangle_{\mathrm{Z}} h_{1}=0, \\
& h_{r}^{4 / N_{r}} \triangle_{\mathrm{Y}_{2}} h_{s}+\triangle_{\mathrm{Z}} h_{s}=0 .
\end{aligned}
$$

The function $h_{r}$ can depend on the coordinate $x^{\mu}$ only if $N_{r}=4$. We can also choose the solution in which the $p_{s}$-brane part depends on $x^{\mu}$. Then, we have

$$
\begin{aligned}
& R_{\mu \nu}(\mathrm{X})=0, \quad R_{i j}\left(\mathrm{Y}_{1}\right)=0, \quad R_{m n}\left(\mathrm{Y}_{2}\right)=0, \quad R_{a b}(\mathrm{Z})=0, \\
& h_{r}=h_{r}(y, z), \quad h_{s}=k_{0}(x)+k_{1}(v, z), \quad \partial_{i} h_{r} \partial_{m} h_{s}=0, \\
& D_{\mu} D_{\nu} k_{0}=0, \quad\left(1-\frac{4}{N_{s}}\right) \partial_{\mu} k_{0} \partial_{\nu} k_{0}=0, \quad h_{r}^{4 / N_{r}} \triangle_{\mathrm{Y}_{2}} k_{1}+\triangle_{\mathrm{Z}} k_{1}=0, \\
& h_{s}^{4 / N_{s}} \triangle_{\mathrm{Y}_{1}} h_{r}+\triangle_{\mathrm{Z}} h_{r}=0 .
\end{aligned}
$$

It is clear that there is no solution for $k_{0}(x)$ such as $\partial_{\mu} h_{s} \neq 0$ unless $N_{s}=4$. If $F_{\left(p_{r}+2\right)}=0$ and $F_{\left(p_{s}+2\right)}=0$, the functions $h_{1}$ and $k_{1}$ become trivial, and the $D$-dimensional spacetime is no longer warped [23,24]. Moreover, the Eq. (18b) $\partial_{i} h_{r} \partial_{m} h_{s}=0$ implies the following two cases:

(i) Two branes are delocalized, which are localized only along the overall transverse directions.

(ii) One brane is completely localized on the other brane which is localized only along the overall transverse directions.

As a special example, let us consider the case

$$
\begin{aligned}
& q_{\mu \nu}=\eta_{\mu \nu}, \quad \gamma_{i j}=\delta_{i j}, \quad w_{m n}=\delta_{m n}, \\
& u_{a b}=\delta_{a b}, \quad N_{r}=N_{s}=4, \quad h_{s}=h_{s}(z),
\end{aligned}
$$

where $\eta_{\mu \nu}$ is the $(p+1)$-dimensional Minkowski metric and $\delta_{i j}, \delta_{m n}, \delta_{a b}$ are the $\left(p_{s}-p\right)-,\left(p_{r}-p\right)$-, and $\left(D+p-p_{r}-p_{s}-1\right)$-dimensional Euclidean metrics, respectively. This means that both branes have physically the same total amount of charge. Since the function $h_{0}$ obeys the equation $\partial_{\mu} \partial_{\nu} h_{0}=0$, we can easily get the solution

$$
h_{0}(x)=A_{\mu} x^{\mu}+B
$$

where $A_{\mu}$ and $B$ are constants. On the other hand, the functions $h_{1}$ and $h_{s}$ satisfy the coupled partial differential equations

$$
h_{s} \triangle_{\mathrm{Y}_{1}} h_{1}+\triangle_{\mathrm{Z}} h_{1}=0, \quad \triangle_{\mathrm{Z}} h_{s}=0 .
$$

The harmonic function $h_{s}$ that satisfies the second differential equation in (18d) has the form

$$
h_{s}=1+\sum_{l} \frac{M_{l}}{\left|z-z_{\ell}\right|^{d_{z}-2}},
$$

where $d_{z} \equiv D+p-p_{r}-p_{s}-1$, and $z_{l}^{a}$ are locations of the $l$-th $p_{s}$-brane with charge $M_{l}$. We will mainly discuss the case in which the $p_{s}$-branes coincide at the same location in the overall transverse directions. Now we choose the following form of the harmonic function $h_{s}$ :

$$
h_{s}(z)=\frac{M}{\left|z-z_{0}\right|^{d_{z}-2}},
$$

where $M$ is constant, $z_{0}$ is the location of the stack of $p_{s}$-branes. It is not so easy to find solutions for the harmonic function $h_{1}$ in the case where each of the $p_{s}$-branes are located at different points along the $z$-directions.

If the dimensionality of the overall transverse space is $d_{z} \neq 2$ and $d_{z} \neq 4$, the equation Eq. (22) can be solved as [18]

$$
h_{1}(y, z)=1+\frac{M_{\ell}}{\left[\left|\boldsymbol{y}-\boldsymbol{y}_{\ell}\right|^{2}+\frac{4 M}{\left(4-d_{z}\right)^{2}}\left|z-z_{0}\right|^{4-d_{z}}\right]^{1 / 2\left(p_{s}-p-1+d_{z} /\left(4-d_{z}\right)\right)}},
$$




\section{MASATO MINAMITSUJI AND KUNIHITO UZAWA}

where $M_{\ell}$ is constant. Hence, the functions $h_{r}$ and $h_{s}$ can be written explicitly as

$$
\begin{aligned}
h_{r}(x, y, z) & =A_{\mu} x^{\mu}+B+\sum_{\ell} \frac{M_{\ell}}{\left[\left|\boldsymbol{y}-\boldsymbol{y}_{\ell}\right|^{2}+\frac{4 M}{\left(4-d_{z}\right)^{2}}\left|z-z_{0}\right|^{4-d_{z}}\right]^{1 / 2\left(p_{s}-p-1+d_{z} /\left(4-d_{z}\right)\right)}}, \\
h_{s}(z) & =\frac{M}{\left|z-z_{0}\right|^{d_{z}-2}},
\end{aligned}
$$

where $A_{\mu}, B, M_{\ell}$ and $M$ are constant parameters, and $\boldsymbol{y}_{\ell}$ and $z_{0}$ are constant vectors representing the positions of the branes. Since the functions coincide, the locations of the branes will also coincide. There are curvature singularities at $h_{r}=0$ or $h_{s}=0$ in the $D$-dimensional metric (5). Moreover, we have a singularity at $z=z_{0}$ unless the dilaton is trivial.

In the case of $d_{z}=2$, we have

$$
\begin{aligned}
h_{r}(x, y, z)= & A_{\mu} x^{\mu}+B \\
& +\sum_{\ell} \frac{M_{\ell}}{\left[\left|\boldsymbol{y}-\boldsymbol{y}_{\ell}\right|^{2}+M\left|z-z_{0}\right|^{2}\right]^{1 / 2\left(p_{s}-p+1\right)}},
\end{aligned}
$$

$$
h_{s}(z)=M \ln \left|z-z_{0}\right| .
$$

For $d_{z}=4$, the solution of Eq. (22) can be written by

$$
\begin{aligned}
h_{r}(x, y, z)= & A_{\mu} x^{\mu}+B+\sum_{\ell} M_{\ell} \ln \left[\left|\boldsymbol{y}-\boldsymbol{y}_{\ell}\right|^{2}\right. \\
& \left.-\left(p_{s}-p\right) M\left|z-z_{0}\right|\right], \\
h_{s}(z)= & \frac{M}{\left|z-z_{0}\right|^{2}} .
\end{aligned}
$$

We note that the solutions (27) and (28) have curvature singularities not only at $h_{r}=0$ but also at the infinity due to the logarithmic spatial dependence of the metric. There is also a singularity at $z=z_{0}$ if the dilaton is nontrivial.

One can easily get the solution for $\partial_{\mu} h_{r}=0$ and $\partial_{\mu} h_{s} \neq 0$ if the roles of $\mathrm{Y}_{1}$ and $\mathrm{Y}_{2}$ are exchanged. The solution of field equations for $d_{z} \neq 2$ and $d_{z} \neq 4$ is thus expressed as

$$
\begin{aligned}
h_{s}(x, v, z) & =A_{\mu} x^{\mu}+B+\sum_{\ell} \frac{M_{\ell}}{\left[\left|\boldsymbol{v}-\boldsymbol{v}_{\ell}\right|^{2}+\frac{4 M}{\left(4-d_{z}\right)^{2}}\left|z-z_{0}\right|^{4-d_{z}}\right]^{1 / 2\left(p_{r}-p-1+d_{z} /\left(4-d_{z}\right)\right)}}, \\
h_{r}(z) & =\frac{M}{\left|z-z_{0}\right|^{d_{z}-2}} .
\end{aligned}
$$

For $d_{z}=2$ and $d_{z}=4$, the harmonic functions have logarithmic spatial dependence like (27) and (28).

Let us briefly summarize the intersecting rules in 11dimensional supergravity and in 10-dimensional string theory. For the M-branes in 11-dimensional supergravity, there is 4-form field strength without dilaton, the intersection rule $\chi=0$ gives

$$
p=\frac{\left(p_{r}+1\right)\left(p_{s}+1\right)}{9}-1,
$$

where $p$ denotes the number of overlapping dimensions of the $p_{r}$ and $p_{s}$ branes. Then we get the intersections involving the M2 and M5-branes [2,4,20,22]

$$
\mathrm{M} 2 \cap \mathrm{M} 2=0, \quad \mathrm{M} 2 \cap \mathrm{M} 5=1, \quad \mathrm{M} 5 \cap \mathrm{M} 5=3 .
$$

For the ten-dimensional string theory, the couplings to dilaton for the RR-charged D-branes are given by

$$
\epsilon_{r} c_{r}=\frac{1}{2}\left(3-p_{r}\right), \quad \epsilon_{s} c_{s}=\frac{1}{2}\left(3-p_{s}\right) .
$$

The condition $\chi=0$ then gives

$$
p=\frac{1}{2}\left(p_{r}+p_{s}-4\right) \text {. }
$$

The intersections for the D-branes are thus given by $[4,20,22]$

$$
\mathrm{D} p_{r} \cap \mathrm{D} p_{s}=\frac{1}{2}\left(p_{r}+p_{s}\right)-2 .
$$

We finally consider the intersections for NS-branes. The parameters $c_{r}$ for fundamental string (F1) and solitonic 5-brane are $\epsilon_{1} c_{1}=-1$ (for F1) and $\epsilon_{5} c_{5}=1$ (for NS5), respectively. Then the intersections involving the F1 and NS5-branes are [4,20,22]

$$
\begin{aligned}
& \mathrm{F} 1 \cap \mathrm{NS} 5=1, \quad \mathrm{NS} 5 \cap \mathrm{NS} 5=3, \\
& \mathrm{~F} 1 \cap \mathrm{D} \bar{p}=0, \\
& \mathrm{D} \bar{p} \cap \mathrm{NS} 5=\bar{p}-1,1 \leq \bar{p} \leq 6 .
\end{aligned}
$$

There is no solution for the F1-F1 and D0-NS5 intersecting brane systems because the numbers of space dimensions for each pairwise overlap are negative by the intersection rule.

\section{B. The intersection of $p$-brane and KK-monopole system}

Now we discuss the dynamical intersecting brane solutions including KK-monopoles in $D$ dimensions. The 
$p$-branes we have described above carry a charge in $D$-dimensions. The Kaluza-Klein (KK) charged objects are also in general branes living in the compactified space-time and carrying a electric or magnetic charge with respect to the 2-form field strength generated by dimensional reduction in $D$-dimensional theory. If after compactification on one direction the space-time dimension is $D$, we then have an electric KK 0-brane and a magnetic KK $(D-4)$-brane. These two objects correspond, in the $(D+1)$-dimensional uncompactified spacetime to configurations where the only nontrivial field is the metric, and which are identified, respectively, to a KKwave and a KK-monopole. The metric in the uncompactified space necessarily has nontrivial off-diagonal terms. In this section, we discuss the KK-monopole and summarize those objects. We extend our brane solutions to the cases with waves next section.

We will start from the $D$-dimensional theory, for which the action in the Einstein frame contains the metric $g_{M N}$, the dilaton $\phi$, and the antisymmetric tensor field of rank $(p+2), F_{(p+2)}$

$$
\begin{aligned}
S= & \frac{1}{2 \kappa^{2}} \int\left[R * \mathbf{1}_{D}-\frac{1}{2} d \phi \wedge * d \phi\right. \\
& \left.-\frac{1}{2 \cdot(p+2) !} \mathrm{e}^{\epsilon c \phi} F_{(p+2)} \wedge * F_{(p+2)}\right],
\end{aligned}
$$

where $\kappa^{2}$ is the $D$-dimensional gravitational constant, $*$ is the Hodge operator in the $D$-dimensional space-time, $F_{(p+2)}$ is the $(p+2)$-form field strength, and $c, \epsilon$ are constants given by

$$
\begin{aligned}
c^{2} & =N-\frac{2(p+1)(D-p-3)}{D-2}, \\
\epsilon & = \begin{cases}+ & \text { if } p-\text { brane is electric } \\
- & \text { if } p-\text { brane is magnetic }\end{cases}
\end{aligned}
$$

Here $N$ is a constant. The field strength $F_{(p+2)}$ is given by the $(p+1)$-form gauge potential $A_{(p+1)}$

$$
F_{(p+2)}=d A_{(p+1)} \text {. }
$$

The field equations are given by

$$
\begin{aligned}
& R_{M N}=\frac{1}{2} \partial_{M} \phi \partial_{N} \phi+\frac{1}{2 \cdot(p+2) !} \mathrm{e}^{\epsilon c \phi}\left[(p+2) F_{M A_{2} \cdots A_{p+2}} F_{N}^{A_{2} \cdots A_{p+2}}-\frac{p+1}{D-2} g_{M N} F_{(p+2)}^{2}\right], \\
& d * d \phi-\frac{\epsilon c}{2 \cdot(p+2) !} \mathrm{e}^{\epsilon c \phi} F_{(p+2)} \wedge * F_{(p+2)}=0, \\
& d\left[\mathrm{e}^{\epsilon c \phi} * F_{(p+2)}\right]=0 .
\end{aligned}
$$

We assume that the $D$-dimensional metric takes the form

$$
\begin{aligned}
d s^{2}= & h^{a}(x, y, z) q_{\mu \nu}(\mathrm{X}) d x^{\mu} d x^{\nu}+h^{b}(x, y, z) \\
& \times\left[\gamma_{i j}(\mathrm{Y}) d y^{i} d y^{j}+h_{k}(x, z) u_{a b}(\mathrm{Z}) d z^{a} d z^{b}\right. \\
& \left.+h_{k}^{-1}(x, z)\left(d v+A_{a} d z^{a}\right)^{2}\right],
\end{aligned}
$$

where $q_{\mu \nu}$ is the $(p+1)$-dimensional metric which depends only on the $(p+1)$-dimensional coordinates $x^{\mu}, \gamma_{i j}$ is the $\left(D-p-d_{z}-2\right)$-dimensional metric which depends only on the $\left(D-p-d_{z}-2\right)$-dimensional coordinates $y^{i}$, and finally $u_{a b}$ is the $d_{z}$-dimensional metric which depends only on the $d_{z}$-dimensional coordinates $z^{a}$. The parameters $a$ and $b$ in the metric (40) are given by

$$
a=-\frac{4(D-p-3)}{N(D-2)}, \quad b=\frac{4(p+1)}{N(D-2)} .
$$

The brane configuration is given in Table II. The $D$-dimensional metric (40) implies that the solutions are characterized by two functions, $h$ and $h_{k}$, which depend on the coordinates transverse to the brane as well as the world volume coordinate.

We also assume that the scalar field $\phi$ and the gauge

\begin{tabular}{|c|c|c|c|c|c|c|c|c|c|c|c|c|}
\hline Case & & 0 & 1 & $\cdots$ & $p$ & $p+1$ & $\cdots$ & $D-d_{z}-2$ & $D-d_{z}-1$ & $D-d_{z}$ & $\cdots$ & $D-1$ \\
\hline \multirow{3}{*}{$p$-KK } & $p$ & $\circ$ & $\circ$ & $\circ$ & $\circ$ & & & & & & & \\
\hline & KK & $\circ$ & $\circ$ & $\circ$ & $\circ$ & $\circ$ & $\circ$ & $\circ$ & & $A_{1}$ & $\cdots$ & $A_{d_{z}}$ \\
\hline & $x^{N}$ & $t$ & $x^{1}$ & $\cdots$ & $x^{p}$ & $y^{1}$ & $\cdots$ & $y^{D-p-d_{z}-2}$ & $v$ & $z^{1}$ & $\cdots$ & $z^{d_{z}^{v}}$ \\
\hline
\end{tabular}
field strength $F_{(p+2)}$ are given by

$$
\begin{aligned}
\mathrm{e}^{\phi} & =h^{2 \epsilon c / N}, \\
F_{(p+2)} & =\frac{2}{\sqrt{N}} d\left[h^{-1}(x, y, z)\right] \wedge \Omega(\mathrm{X}),
\end{aligned}
$$

where $\Omega(\mathrm{X})$ denotes the volume $(p+1)$-form

$$
\Omega(\mathrm{X})=\sqrt{-q} d x^{0} \wedge d x^{1} \wedge \cdots \wedge d x^{p} .
$$

Here, $q$ is the determinant of the metric $q_{\mu \nu}$.

First we consider the Einstein Eq. (39a). Using the assumptions (40) and (42), the Einstein equations are given by

TABLE II. Intersections of $p$-brane and KK-monopole in the metric (40). 


$$
\begin{aligned}
& R_{\mu \nu}(\mathrm{X})-\frac{4}{N} h^{-1} D_{\mu} D_{\nu} h+\frac{2}{N}\left(1-\frac{4}{N}\right) h^{-1} \partial_{\mu} \ln h \partial_{\nu} \ln h-\frac{a}{2} q_{\mu \nu}\left[h^{-1} \triangle_{\mathrm{X}} h+q^{\rho \sigma} \partial_{\rho} \ln h\left\{\left(\frac{4}{N}-1\right) \partial_{\sigma} \ln h\right.\right. \\
& \left.\left.+\frac{1}{2}\left(d_{z}-1\right) \partial_{\sigma} \ln h_{k}\right\}\right]-\frac{a}{2} q_{\mu \nu} h^{-1-(4 / N)} \triangle_{\mathrm{Y}} h-\frac{a}{2} q_{\mu \nu} h^{-1-(4 / N)} h_{k}^{-1} \triangle_{\mathrm{Z}} h \\
& +\left[\frac{a}{2}-\frac{1}{N}\left(d_{z}+1\right)\right] \partial_{\mu} \ln h \partial_{\nu} \ln h_{k}+\frac{1}{4}\left(d_{z}-3\right)\left[\partial_{\mu} \ln h_{k} \partial_{\nu} \ln h_{k}-h^{-4 / N} h_{k}^{-1} q_{\mu \nu} u^{a b} \partial_{a} \ln h \partial_{b} \ln h_{k}\right]=0, \\
& \frac{2}{N}\left[h^{-1} \partial_{\mu} \partial_{i} h+\frac{1}{2}\left(d_{z}-1\right) \partial_{\mu} \ln h_{k} \partial_{i} \ln h\right]=0 \text {, } \\
& h^{-1} \partial_{\mu} \partial_{a} h+\frac{1}{2}\left(d_{z}-2\right) h_{k}^{-1} \partial_{\mu} \partial_{a} h_{k}-\frac{1}{N}\left(d_{z}-3\right) \partial_{\mu} \ln h \partial_{a} \ln h_{k}=0 \text {, } \\
& R_{i j}(\mathrm{Y})-\frac{b}{2} h^{4 / N} \gamma_{i j}\left[h^{-1} \triangle_{\mathrm{X}} h+q^{\rho \sigma} \partial_{\rho} \ln h\left\{\left(\frac{4}{N}-1\right) \partial_{\sigma} \ln h+\frac{1}{2}\left(d_{z}-1\right) \partial_{\sigma} \ln h_{k}\right\}\right]-\frac{b}{2} h^{-1} \gamma_{i j}\left(\triangle_{\mathrm{Y}} h+h_{k}^{-1} \triangle_{\mathrm{Z}} h\right) \\
& -\frac{b}{4}\left(d_{z}-3\right) \gamma_{i j} h_{k}^{-1} u^{a b} \partial_{a} \ln h \partial_{b} \ln h_{k}=0 \text {, } \\
& R_{a b}(\mathrm{Z})-\frac{b}{2} h^{4 / N} h_{k}\left(u_{a b}+h_{k}^{-2} A_{a} A_{b}\right)\left[h^{-1} \triangle_{\mathrm{X}} h+q^{\rho \sigma} \partial_{\rho} \ln h\left\{\left(\frac{4}{N}-1\right) \partial_{\sigma} \ln h+\frac{1}{2}\left(d_{z}-1\right) \partial_{\sigma} \ln h_{k}\right\}\right] \\
& -\frac{b}{2} h^{-1} h_{k}\left(u_{a b}+h_{k}^{-2} A_{a} A_{b}\right)\left(\triangle_{\mathrm{Y}} h+h_{k}^{-1} \triangle_{\mathrm{Z}} h\right)-\frac{1}{2} h_{k}^{-1}\left(u_{a b}-h_{k}^{-2} A_{a} A_{b}\right) \triangle_{\mathrm{Z}} h_{k}-\frac{1}{2}\left(d_{z}-3\right) h_{k}^{-1} D_{a} D_{b} h_{k} \\
& -\frac{b}{4}\left(d_{z}-3\right)\left(u_{a b}+h_{k}^{-2} A_{a} A_{b}\right) u^{a b} \partial_{a} \ln h \partial_{b} \ln h_{k}=0 \text {, } \\
& h^{4 / N} h_{k}^{-1}\left[h^{-1} \triangle_{\mathrm{X}} h-h_{k}^{-1} \triangle_{\mathrm{X}} h_{k}+b\left(1-\frac{4}{N}\right) q^{\rho \sigma} \partial_{\rho} \ln h \partial_{\sigma} \ln h-\left\{a+\frac{b}{2}\left(d_{z}-3\right)\right\} q^{\rho \sigma} \partial_{\rho} \ln h \partial_{\sigma} \ln h_{k}\right. \\
& \left.+\frac{1}{2}\left(d_{z}-3\right) q^{\rho \sigma} \partial_{\rho} \ln h_{k} \partial_{\sigma} \ln h_{k}\right]-b\left(h h_{k}\right)^{-1}\left[\triangle_{\mathrm{Y}} h+h_{k}^{-1} \triangle_{\mathrm{Z}} h+\frac{1}{2}\left(d_{z}-3\right) u^{a b} \partial_{a} \ln h_{k}\left(b \partial_{b} \ln h-\partial_{b} \ln h_{k}\right)\right]=0 \text {, }
\end{aligned}
$$

where $D_{\mu}$ is the covariant derivative with respect to the metric $q_{\mu \nu}$, and we assumed $d h_{k}=*_{\mathrm{Z}} d A_{(1)}$, and $\triangle_{\mathrm{X}}, \triangle_{\mathrm{Y}}$, $\triangle_{\mathrm{Z}}$ are the Laplace operators on $\mathrm{X}, \mathrm{Y}, \mathrm{Z}$ space, and $R_{\mu \nu}(\mathrm{X}), R_{i j}(\mathrm{Y}), R_{a b}(\mathrm{Z})$ are the Ricci tensors associated with the metrics $q_{\mu \nu}(\mathrm{X}), \gamma_{i j}(\mathrm{Y}), u_{a b}(\mathrm{Z})$, respectively. For $d_{z}=3$, we see from Eqs. (44b) and (44c) that the warp factors $h$ must take the form

$$
\begin{aligned}
& h=h_{0}(x)+h_{1}(y, z), \quad h_{k}=h_{k}(z), \\
& \text { for } \partial_{\mu} h_{k}=0 \text { and } N=4 \text {. }
\end{aligned}
$$

Now we consider the case $\partial_{\mu} h_{k}=0$ and $N=4$. The components of the Einstein Eqs. (44) are rewritten as

$$
\begin{aligned}
& R_{\mu \nu}(\mathrm{X})-h^{-1} D_{\mu} D_{\nu} h-\frac{a}{2} q_{\mu \nu}\left(h^{-1} \triangle_{\mathrm{X}} h+q^{\rho \sigma} \partial_{\rho} \ln h \partial_{\sigma} \ln h_{k}\right)+\frac{a}{2}(a-2) \partial_{\mu} \ln h \partial_{\nu} \ln h_{k} \\
& \quad-\frac{a}{2} q_{\mu \nu} h^{-2}\left(\triangle_{\mathrm{Y}} h+h_{k}^{-1} \triangle_{\mathrm{Z}} h\right)=0 \\
& R_{i j}(\mathrm{Y})-\frac{b}{2} h \gamma_{i j}\left(h^{-1} \triangle_{\mathrm{X}} h+q^{\rho \sigma} \partial_{\rho} \ln h \partial_{\sigma} \ln h_{k}\right)-\frac{b}{2} h^{-1} \gamma_{i j}\left(\triangle_{\mathrm{Y}} h+h_{k}^{-1} \triangle_{\mathrm{Z}} h\right)=0 \\
& R_{a b}(\mathrm{Z})-\frac{b}{2} h h_{k}\left(u_{a b}+h_{k}^{-2} A_{a} A_{b}\right)\left(h^{-1} \triangle_{\mathrm{X}} h+q^{\rho \sigma} \partial_{\rho} \ln h \partial_{\sigma} \ln h_{k}\right)-\frac{b}{2} h^{-1} h_{k}\left(u_{a b}+h_{k}^{-2} A_{a} A_{b}\right)\left(\triangle_{\mathrm{Y}} h+h_{k}^{-1} \triangle_{\mathrm{Z}} h\right) \\
& \quad-\frac{1}{2} h_{k}^{-1}\left(u_{a b}-h_{k}^{-2} A_{a} A_{b}\right) \triangle_{\mathrm{Z}} h_{k}=0, \\
& h_{k}^{-1}\left(\triangle_{\mathrm{X}} h-h h_{k}^{-1} \triangle_{\mathrm{X}} h_{k}\right)-b\left(h h_{k}\right)^{-1}\left(\triangle_{\mathrm{Y}} h+h_{k}^{-1} \triangle_{\mathrm{Z}} h\right)=0 .
\end{aligned}
$$

Next we consider the gauge field Eqs. (39c). Under the assumption (42b), we find

$d\left[h_{k} \partial_{i} h\left(*_{\mathrm{Y}} d y^{i}\right) \wedge \Omega(\mathrm{Z})+\partial_{a} h\left(*_{\mathrm{Z}} d z^{a}\right) \wedge \Omega(\mathrm{Y})\right] \wedge d v=0$, where $*_{\mathrm{Y}}$, $*_{\mathrm{Z}}$ denote the Hodge operator on $\mathrm{Y}, \mathrm{Z}$, respectively, and $\Omega(\mathrm{Y}), \Omega(\mathrm{Z})$ denote the volume $(D-p-$ $\left.d_{z}-2\right)$-, $d_{z}$-form respectively:

$$
\begin{aligned}
& \Omega(\mathrm{Y})=\sqrt{\gamma} d y^{1} \wedge d y^{2} \wedge \cdots \wedge d y^{D-p-d_{z}-2} \\
& \Omega(\mathrm{Z})=\sqrt{u} d z^{1} \wedge d z^{2} \wedge \cdots \wedge d z^{d_{z}} .
\end{aligned}
$$


Then, the Eq. (47) gives

$$
\begin{aligned}
h_{k} \triangle_{\mathrm{Y}} h+\triangle_{\mathrm{Z}} h & =0, \\
\partial_{\mu} \partial_{i} h+\partial_{\mu} h_{k} \partial_{i} h & =0, \\
\partial_{\mu} \partial_{a} h & =0,
\end{aligned}
$$

where $\triangle_{Y}, \triangle_{Z}$ are the Laplace operators on the space of $\mathrm{Y}$, $\mathrm{Z}$, respectively.

Finally we should consider the scalar field equation. Substituting Eqs. (42) and (45) into Eq. (39b), we obtain

$$
\begin{aligned}
h^{4 / N} & {\left[h^{-1} \triangle_{\mathrm{X}} h_{0}+q^{\rho \sigma} \partial_{\rho} \ln h \partial_{\sigma} \ln h_{k}\right.} \\
& -\left(1-\frac{4}{N}\right) q^{\rho \sigma} \partial_{\rho} \ln h \partial_{\sigma} \ln h \\
& \left.+h^{-1}\left(\triangle_{\mathrm{Y}} h_{1}+h_{k}^{-1} \triangle_{\mathrm{Z}} h_{1}\right)\right]=0 .
\end{aligned}
$$

Thus, for $N=4$, the warp factor $h$ should satisfy the equations

$$
\triangle_{\mathrm{X}} h_{0}=0, \quad \partial_{\mu} h_{0} \partial_{\nu} h_{k}=0, \quad \triangle_{\mathrm{Y}} h_{1}+h_{k}^{-1} \triangle_{\mathrm{Z}} h_{1}=0 .
$$

Combining these, we find that these field equations lead to

$R_{\mu \nu}(\mathrm{X})=0, \quad R_{i j}(\mathrm{Y})=0, \quad R_{a b}(\mathrm{Z})=0$,

$h=h_{0}(x)+h_{1}(z)$,

$D_{\mu} D_{\nu} h_{0}=0, \quad \partial_{\mu} h_{0} \partial_{\nu} h_{k}=0$,

$$
\begin{aligned}
& \left(1-\frac{4}{N}\right) q^{\rho \sigma} \partial_{\rho} \ln h \partial_{\sigma} \ln h=0, \quad \triangle_{\mathrm{Z}} h_{1}=0, \\
& h_{k}^{4 / N} \triangle_{\mathrm{Y}} h_{1}+\triangle_{\mathrm{Z}} h_{1}=0 .
\end{aligned}
$$

The function $h$ can depend on the coordinate $x$ only if $N=4$. If $F_{(p+2)}=0$, the function $h_{1}$ becomes trivial.

As a special example, let us consider the case

$$
\begin{gathered}
q_{\mu \nu}=\eta_{\mu \nu}, \quad \gamma_{i j}=\delta_{i j}, \quad u_{a b}=\delta_{a b}, \\
N=4, \quad h_{k}=h_{k}(z),
\end{gathered}
$$

where $\eta_{\mu \nu}$ is the $(p+1)$-dimensional Minkowski metric and $\delta_{i j}, \delta_{a b}$ are the $(5-p)$-, three-dimensional Euclidean metrics, respectively. The solution for $h$ and $h_{k}$ can be obtained explicitly as

$$
\begin{aligned}
h(x, y, z)= & A_{\mu} x^{\mu}+\tilde{c} \\
& +\sum_{\ell} \frac{M_{\ell}}{\left[\left|\boldsymbol{y}-\boldsymbol{y}_{\ell}\right|^{2}+4 M\left|z-z_{0}\right|\right]^{1 / 2(D-p-3)}}, \\
h_{k}(z)= & \frac{M}{\left|z-z_{0}\right|},
\end{aligned}
$$

where $A_{\mu}, \tilde{c}, M_{\ell}$ and $M$ are constant parameters, and $\boldsymbol{y}_{\ell}$ and $z_{0}$ are constant vectors representing the positions of the branes. Since the functions coincide, the locations of the branes will also coincide. The $D$-dimensional metric (40) exists for $h>0$ and has curvature singularities at $h=0$.

\section{The intersection of $p$-brane and plane wave system}

Let us next consider the solutions with the plane wave. One can obtain the electric 0-brane and the magnetic $(D-5)$-brane solutions in $(D-1)$-dimensional spacetime because the dimensional reduction generates the KaluzaKlein charge in the 2-form field strengths. After we lift up those solutions by one dimension, we obtain the plane wave solutions in $D$-dimensions. We briefly discuss the plane wave solution in this section.

Now we look for solution whose spacetime metric has the form

$$
\begin{aligned}
d s^{2}= & h^{a_{w}}(t, z)\left[-d t^{2}+d x^{2}+\left\{h_{w}(t, y, z)-1\right\}(d t-d x)^{2}\right. \\
& \left.+\gamma_{i j}(\mathrm{Y}) d y^{i} d y^{j}\right]+h^{b_{w}}(t, z) u_{a b}(\mathrm{Z}) d z^{a} d z^{b}
\end{aligned}
$$

where $\gamma_{i j}$ is the $(p-1)$-dimensional metric which depends only on the $(p-1)$-dimensional coordinates $y^{i}$, and finally $u_{a b}$ is the $(D-p-1)$-dimensional metric which depends only on the $(D-p-1)$-dimensional coordinates $z^{a}$. The parameters $a$ and $b$ in the metric (55) are given by

$$
a_{w}=-\frac{4(D-p-3)}{N(D-2)}, \quad b_{w}=\frac{4(p+1)}{N(D-2)} .
$$

We show the brane configuration in Table III.

We also assume that the scalar field $\phi$ and the gauge field strength $F_{(p+2)}$ are given by

$$
\begin{aligned}
\mathrm{e}^{\phi} & =h^{2 \epsilon c / N} \\
F_{(p+2)} & =\frac{2}{\sqrt{N}} d\left[h^{-1}(t, z)\right] \wedge d t \wedge d x \wedge \Omega(\mathrm{Y}),
\end{aligned}
$$

where $\Omega(\mathrm{Y})$ denotes the volume ( $p-1)$-form:

$$
\Omega(\mathrm{Y})=\sqrt{\gamma} d y^{1} \wedge d y^{2} \wedge \cdots \wedge d y^{p-1} .
$$

Here, $\gamma$ is the determinant of the metric $\gamma_{i j}$.

First, we consider the Einstein Eqs. (39a). Using the assumptions (55) and (57), the Einstein equations are given by

TABLE III. Intersections of $p$-brane and plane wave in the metric (55).

\begin{tabular}{lccccccccc}
\hline \hline Case & & 0 & 1 & 2 & $\cdots$ & $p$ & $p+1$ & $\cdots$ & $D-1$ \\
\hline & $p$ & $\circ$ & $\circ$ & $\circ$ & $\circ$ & $\circ$ & & & \\
$p-\mathrm{W}$ & $\mathrm{W}$ & $\circ$ & & & & & & & \\
& $x^{N}$ & $t$ & $x$ & $y^{1}$ & $\cdots$ & $y^{p-1}$ & $z^{1}$ & $\cdots$ & $z^{D-p-1}$ \\
\hline \hline
\end{tabular}




$$
\begin{aligned}
& {\left[a_{w}\left(2-h_{w}\right)-\frac{8}{N}\right] h^{-1} \partial_{t}^{2} h+\left(2-h_{w}\right) \partial_{t}^{2} h_{w}+\triangle_{\mathrm{Y}} h_{w}+h^{-4 / N} \triangle_{\mathrm{Z}} h_{w}+2\left(2-h_{w}\right) h^{-1-(4 / N)} \triangle_{\mathrm{Z}} h} \\
& \quad-\frac{4}{N}\left(\frac{4}{N}-1\right)\left(\partial_{t} \ln h\right)^{2}-a_{w} h_{w}\left(2-h_{w}\right)\left[\left(\frac{4}{N}-1\right) \partial_{t} \ln h+\partial_{t} \ln h_{w}\right] \partial_{t} \ln h+\frac{4}{N}\left(2-h_{w}\right) \partial_{t} \ln h \partial_{t} h_{w}=0, \\
& \partial_{t} \partial_{i} h_{w}=0, \\
& \partial_{t} \partial_{a} h_{w}+\frac{4}{N} h^{-1} \partial_{t} \partial_{a} h=0, \\
& a_{w} h_{w}^{2} h^{-1} \partial_{t}^{2} h+h_{w} \partial_{t}^{2} h_{w}-\triangle_{\mathrm{Y}} h_{w}-h^{-4 / N} \triangle_{\mathrm{Z}} h_{w}+a_{w} h_{w} h^{-1-4 / N} \triangle_{\mathrm{Z}} h \\
& \quad+a_{w} h_{w}^{2}\left[\left(\frac{4}{N}-1\right) \partial_{t} \ln h+\left(\frac{4}{N}+1\right) \partial_{t} \ln h_{w}\right] \partial_{t} \ln h=0, \\
& R_{i j}(\mathrm{Y})+\frac{a_{w}}{2} h^{-1} h_{w} \gamma_{i j}\left[\partial_{t}^{2} h+\left\{\left(\frac{4}{N}-1\right) \partial_{t} \ln h+\partial_{t} \ln h_{w}\right\} \partial_{t} \ln h\right]-\frac{a_{w}}{2} h^{-1-(4 / N)} \triangle_{\mathrm{Z}} h=0, \\
& R_{a b}(\mathrm{Z})+\frac{a_{w}}{2} h^{4 / N} h_{w} u_{a b}\left[h^{-1} \partial_{t}^{2} h+\left\{\left(\frac{4}{N}-1\right) \partial_{t} \ln h+\partial_{t} \ln h_{w}\right\} \partial_{t} \ln h\right]-\frac{b_{w}}{2} h^{-1} \triangle_{\mathrm{Z}} h=0,
\end{aligned}
$$

where $\triangle_{\mathrm{Y}}, \triangle_{\mathrm{Z}}$ are the Laplace operators on $\mathrm{Y}, \mathrm{Z}$ space, and $R_{i j}(\mathrm{Y}), R_{a b}(\mathrm{Z})$ are the Ricci tensors associated with the metrics $\gamma_{i j}(\mathrm{Y}), u_{a b}(\mathrm{Z})$, respectively.

We see from Eqs. (59b) and (59c) that the warp factors $h$ must take the form

$$
\begin{aligned}
& h=h_{0}(t)+h_{1}(z), \quad h_{w}=h_{w}(y, z), \quad \text { for } \partial_{t} h_{w}=0, \\
& h=h(z), \quad h_{w}=k_{0}(t)+k_{1}(y, z), \quad \text { for } \partial_{t} h=0 .
\end{aligned}
$$

Let us first consider the case $\partial_{t} h_{w}=0$. The components of the Einstein Eqs. (59) are rewritten as

$$
\begin{aligned}
& {\left[a_{w}\left(2-h_{w}\right)-\frac{8}{N}\right] h^{-1} \partial_{t}^{2} h_{0}+\triangle_{\mathrm{Y}} h_{w}+h^{-1} \triangle_{\mathrm{Z}} h_{w}+2\left(2-h_{w}\right) h^{-2} \triangle_{\mathrm{Z}} h_{1}} \\
& \quad-\frac{4}{N}\left(\frac{4}{N}-1\right)\left(\partial_{t} \ln h\right)^{2}-a_{w} h_{w}\left(2-h_{w}\right)\left[\left(\frac{4}{N}-1\right) \partial_{t} \ln h+\partial_{t} \ln h_{w}\right] \partial_{t} \ln h+\frac{4}{N}\left(2-h_{w}\right) \partial_{t} \ln h \partial_{t} h_{w}=0, \\
& a_{w} h_{w}^{2} h^{-1} \partial_{t}^{2} h_{0}-\triangle_{\mathrm{Y}} h_{w}-h^{-1} \triangle_{\mathrm{Z}} h_{w}+a_{w} h_{w} h^{-2} \triangle_{\mathrm{Z}} h_{1}+a_{w} h_{w}^{2}\left[\left(\frac{4}{N}-1\right) \partial_{t} \ln h+\left(\frac{4}{N}+1\right) \partial_{t} \ln h_{w}\right] \partial_{t} \ln h=0, \\
& R_{i j}(\mathrm{Y})+\frac{a_{w}}{2} h^{-1} h_{w} \gamma_{i j}\left[\partial_{t}^{2} h_{0}+\left\{\left(\frac{4}{N}-1\right) \partial_{t} \ln h+\partial_{t} \ln h_{w}\right\} \partial_{t} \ln h\right]-\frac{a_{w}}{2} h^{-2} \triangle_{\mathrm{Z}} h_{1}=0, \\
& R_{a b}(\mathrm{Z})+\frac{a_{w}}{2} u_{a b} h_{w}\left[h^{-1} \partial_{t}^{2} h_{0}+\left\{\left(\frac{4}{N}-1\right) \partial_{t} \ln h+\partial_{t} \ln h_{w}\right\} \partial_{t} \ln h\right]-\frac{b_{w}}{2} h^{-1} \triangle_{\mathrm{Z}} h_{1}=0 .
\end{aligned}
$$

Now we consider the gauge field Eqs. (39c). Under the assumption (57b), we find

$$
d\left[\partial_{a} h\left(*_{\mathrm{Z}} d z^{a}\right)\right]=0,
$$

where $*_{\mathrm{Z}}$ denotes the Hodge operator on $\mathrm{Z}$.

Then, the Eq. (62) gives

$$
\triangle_{\mathrm{Z}} h=0, \quad \partial_{t} \partial_{a} h=0 .
$$

Finally we should consider the scalar field equation. Substituting Eqs. (57) and (60) into Eq. (39b), we obtain

$$
\begin{aligned}
& \frac{2 \epsilon c}{N} h^{-b+4 / N-1} h_{w}\left[\partial_{t}^{2} h_{0}+\left\{\left(\frac{4}{N}-1\right) \partial_{t} \ln h+\partial_{t} \ln h_{w}\right\} \partial_{t} h_{0}\right. \\
& \left.\quad+h_{w} \triangle_{\mathrm{Z}} h_{1}\right]=0
\end{aligned}
$$

where we used (60a). Thus, the warp factor $h$ should satisfy the equations

$$
\begin{aligned}
\partial_{t}^{2} h_{0} & =0, \\
\left(\frac{4}{N}-1\right) \partial_{t} \ln h+\partial_{t} \ln h_{w} & =0, \\
\triangle_{\mathrm{Z}} h_{1} & =0 .
\end{aligned}
$$

Combining these, we find that these field equations lead to

$$
\begin{aligned}
& R_{i j}(\mathrm{Y})=0, \quad R_{a b}(\mathrm{Z})=0, \\
& h=h_{0}(t)+h_{1}(z), \\
& \partial_{t}^{2} h_{0}=0, \quad \partial_{t} h_{0} \partial_{t} h_{w}=0, \\
& \quad\left(\frac{4}{N}-1\right) \partial_{t} \ln h+\partial_{t} \ln h_{w}=0, \quad \triangle_{\mathrm{Z}} h_{1}=0, \\
& h^{4 / N} \triangle_{\mathrm{Y}} h_{w}+\triangle_{\mathrm{Z}} h_{w}=0 .
\end{aligned}
$$

The function $h$ can depend on the coordinate $t$ only if $N=4$. We can also choose the solution in which the function $h_{w}$ depends on $t$. Then, we have 


$$
\begin{aligned}
& R_{i j}(\mathrm{Y})=0, \quad R_{a b}(\mathrm{Z})=0, \\
& h=h(z), \quad h_{w}=k_{0}(t)+k_{1}(y, z), \\
& \partial_{t}^{2} k_{0}=0, \quad h^{4 / N} \triangle_{\mathrm{Y}} k_{1}+\triangle_{\mathrm{Z}} k_{1}=0 .
\end{aligned}
$$

If $F_{(p+2)}=0$, the functions $h_{1}$ become trivial.

As a special example, let us consider the case

$$
\gamma_{i j}=\delta_{i j}, \quad u_{a b}=\delta_{a b}, \quad N=4, \quad h=h(z),
$$

where $\delta_{i j}, \delta_{a b}$ are the $(p-1)$-, $(D-p-1)$-dimensional Euclidean metrics, respectively. This physically means that both branes have the same total amount of charge. The solution for $h$ and $h_{w}$ can be obtained explicitly as

$$
\begin{aligned}
h_{w}(t, z) & =A_{\mu} x^{\mu}+B+\sum_{\ell} \frac{M_{\ell}}{\left[\left|\boldsymbol{y}-\boldsymbol{y}_{\ell}\right|^{2}+\frac{4 M}{(D-p-5)^{2}}\left|z-z_{0}\right|^{-D+p+5}\right]^{1 / 2(p-2-(D-p-1 / D-p-5)}}, \\
h(z) & =\frac{M}{\left|z-z_{0}\right|^{D-p-3}},
\end{aligned}
$$

where $A_{\mu}, B, M_{\ell}$ and $M$ are constant parameters, and $\boldsymbol{y}_{\ell}$ and $z_{0}$ are constant vectors representing the positions of the branes. Since the functions coincide, the locations of the branes will also coincide. Even if the near-brane structure is regular, we expect another type of singularity may appear at $h_{w}=0$ due to the presence of the time dependence. For $c \neq 0$, the $D$-dimensional spacetime has curvature singularities where $z=z_{0}$ since the scalar field diverges there.

\section{THE INTERSECTION OF DYNAMICAL BRANES IN 11-DIMENSIONAL THEORY}

In this section, we apply the above solutions to 11dimensional theory. In this theory, we have a 4-form field strength and no dilaton. The 4-form gives rise to 2- and 5branes, called, respectively, M2 and M5. We also obtain the KK-wave and KK-monopole in 11 dimensions. In particular, KK-wave is called "M-wave" in 11-dimensional theory $[2,25,26]$. The 11-dimensional action which contains the metric $g_{M N}$, and 4-form field strength $F_{(4)}$ is given by

$$
S=\frac{1}{2 \kappa^{2}} \int\left[R * \mathbf{1}-\frac{1}{2 \cdot 4 !} F_{(4)} \wedge * F_{(4)}\right],
$$

where $\kappa^{2}$ is the 11 -dimensional gravitational constant, $*$ is the Hodge operator in the 11-dimensional space-time. The field strengths $F_{(4)}$ is given by the 3 -form gauge potential

$$
F_{(4)}=d C_{(3)} \text {. }
$$

The field equations are given by

$$
\begin{aligned}
R_{M N} & =\frac{1}{2} \partial_{M} \phi \partial_{N} \phi+\frac{1}{2 \cdot 4 !}\left[4 F_{M A B C} F_{N}^{A B C}-\frac{1}{2} g_{M N} F_{(4)}^{2}\right], \\
d\left[* F_{(4)}\right] & =0, \quad d F_{(4)}=0 .
\end{aligned}
$$

In the following, we discuss the dynamical brane solution for all the possible combinations of intersecting brane pairs in the 11-dimensional theory.

\section{A. The intersection of two M2-branes}

Let us consider the solution of two M2-branes. We assume that the 11-dimensional metric is written by

$$
\begin{aligned}
d s^{2}= & h_{2}^{-2 / 3}(t, y, z) \bar{h}_{2}^{-2 / 3}(z)\left[-d t^{2}+h_{2}(t, y, z) \gamma_{i j}\left(\mathrm{Y}_{1}\right) d y^{i} d y^{j}\right. \\
& +\bar{h}_{2}(z) w_{m n}\left(\mathrm{Y}_{2}\right) d v^{m} d v^{n} \\
& \left.+h_{2}(t, y, z) \bar{h}_{2}(z) u_{a b}(\mathrm{Z}) d z^{a} d z^{b}\right],
\end{aligned}
$$

where $\gamma_{i j}$ is the two-dimensional metric which depends only on the two-dimensional coordinates $y^{i}, w_{m n}$ is the two-dimensional metric which depends only on the two-dimensional coordinates $v^{m}$, and finally $u_{a b}$ is the six-dimensional metric which depends only on the sixdimensional coordinates $z^{a}$.

We also assume that the gauge field strength $F_{(4)}$ is given by

$$
F_{(4)}=d\left[h_{2}^{-1}(t, y, z) d t \wedge \Omega\left(\mathrm{Y}_{2}\right)+\bar{h}_{2}^{-1}(z) d t \wedge \Omega\left(\mathrm{Y}_{1}\right)\right],
$$

where $\Omega\left(\mathrm{Y}_{1}\right)$ and $\Omega\left(\mathrm{Y}_{2}\right)$ denote the volume 2-form and 2form, respectively

$$
\begin{aligned}
& \Omega\left(\mathrm{Y}_{1}\right)=\sqrt{\gamma} d y^{1} \wedge d y^{2}, \\
& \Omega\left(\mathrm{Y}_{2}\right)=\sqrt{w} d v^{1} \wedge d v^{2} .
\end{aligned}
$$

Here, $\gamma$ and $w$ are the determinant of the metric $\gamma_{i j}$, and $w_{m n}$, respectively.

In terms of ansatz for fields (73) and (74), the field equations lead to

$$
\begin{aligned}
& R_{i j}\left(\mathrm{Y}_{1}\right)=0, \quad R_{m n}\left(\mathrm{Y}_{2}\right)=0, \quad R_{a b}(\mathrm{Z})=0, \\
& h_{2}=h_{0}(t)+h_{1}(y, z), \quad \partial_{t}^{2} h_{0}=0, \\
& \bar{h}_{2} \triangle_{\mathrm{Y}_{1}} h_{1}+\triangle_{\mathrm{Z}} h_{1}=0, \quad \triangle_{\mathrm{Z}} \bar{h}_{2}=0,
\end{aligned}
$$

where $\triangle_{\mathrm{Y}_{1}}, \triangle_{\mathrm{Z}}$ are the Laplace operators on $\mathrm{Y}_{1}, \mathrm{Z}$ space, and $R_{i j}\left(\mathrm{Y}_{1}\right), R_{m n}\left(\mathrm{Y}_{2}\right), R_{a b}(\mathrm{Z})$ are the Ricci tensors associated with the metrics $\gamma_{i j}\left(\mathrm{Y}_{1}\right), w_{m n}\left(\mathrm{Y}_{2}\right), u_{a b}(\mathrm{Z})$, respectively. As a special example, let us consider the case 
MASATO MINAMITSUJI AND KUNIHITO UZAWA

$$
\gamma_{i j}=\delta_{i j}, \quad w_{m n}=\delta_{m n}, \quad u_{a b}=\delta_{a b},
$$

where $\delta_{i j}, \delta_{m n}, \delta_{a b}$ are the two-, two-, six-dimensional Euclidean metrics, respectively. The solution for $h_{2}$ and $\bar{h}_{2}$ can be obtained explicitly as

$$
\begin{aligned}
h_{2}(t, y, z) & =\bar{c} t+\tilde{c}+\sum_{\ell} M_{\ell}\left[\left|\boldsymbol{y}-\boldsymbol{y}_{\ell}\right|^{2}+M\left|z-z_{0}\right|^{-2}\right], \\
\bar{h}_{2}(z) & =\frac{M}{\left|z-z_{0}\right|^{4}},
\end{aligned}
$$

where $\bar{c}, \tilde{c}, M_{\ell}$ and $M$ are constant parameters, and $\boldsymbol{y}_{\ell}$ and $z_{0}$ are constant vectors representing the positions of the branes. Since the functions coincide, the locations of the branes will also coincide. If we delocalize along $n$ of the overall transverse directions, the harmonic functions take the following form:

$$
\begin{aligned}
h_{2}(t, y, z) & =\bar{c} t+\tilde{c}+\sum_{\ell} \frac{M_{\ell}}{\left[\left|\boldsymbol{y}-\boldsymbol{y}_{\ell}\right|^{2}+\frac{4 M}{(n-2)^{2}}\left|z-z_{0}\right|^{n-2}\right]^{2 /(n-2)}}, \\
\bar{h}_{2}(z) & =\frac{M}{\left|z-z_{0}\right|^{4-n}} .
\end{aligned}
$$

\section{B. Intersecting M2- and M5-branes}

Next we consider the solution of M2-M5 branes. We assume that the 11-dimensional metric is written by

$$
\begin{aligned}
d s^{2}= & h_{2}^{-2 / 3}(x, y, z) h_{5}^{-1 / 3}(z)\left[q_{\mu \nu}(\mathrm{X}) d x^{\mu} d x^{\nu}\right. \\
& +h_{2}(x, y, z) \gamma_{i j}(\mathrm{Y}) d y^{i} d y^{j}+h_{5}(z) d v^{2} \\
& \left.+h_{2}(x, y, z) h_{5}(z) u_{a b}(\mathrm{Z}) d z^{a} d z^{b}\right]
\end{aligned}
$$

where $q_{\mu \nu}$ is the two-dimensional metric which depends only on the two-dimensional coordinates $x^{\mu}, \gamma_{i j}$ is the four-dimensional metric which depends only on the four-dimensional coordinates $y^{i}$, and finally $u_{a b}$ is the four-dimensional metric which depends only on the four-dimensional coordinates $z^{a}$.

We also assume that the gauge field strength $F_{(4)}$ is given by

$$
\begin{aligned}
F_{(4)}= & d\left[h_{2}^{-1}(x, y, z)\right] \wedge \Omega(\mathrm{X}) \wedge d v \\
& +*\left[d h_{5}^{-1}(z) \Omega(\mathrm{X}) \wedge \Omega(\mathrm{Y})\right],
\end{aligned}
$$

where $\Omega(\mathrm{X})$ and $\Omega(\mathrm{Y})$ denote the volume 2-form and 4-form, respectively

$$
\begin{aligned}
& \Omega(\mathrm{X})=\sqrt{-q} d x^{0} \wedge d x^{1} \\
& \Omega(\mathrm{Y})=\sqrt{\gamma} d y^{1} \wedge d y^{2} \wedge d y^{3} \wedge d y^{4} .
\end{aligned}
$$

Here, $q$ and $\gamma$ are the determinant of the metric $q_{\mu \nu}$ and $\gamma_{i j}$, respectively.

In terms of ansatz for fields (80) and (81), the field equations lead to
PHYSICAL REVIEW D 84, 126006 (2011)

$R_{\mu \nu}(\mathrm{X})=0, \quad R_{i j}(\mathrm{Y})=0, \quad R_{a b}(\mathrm{Z})=0$,

$h_{2}=h_{0}(x)+h_{1}(y, z)$,

$D_{\mu} D_{\nu} h_{0}=0, \quad h_{5} \triangle_{\mathrm{Y}} h_{1}+\triangle_{\mathrm{Z}} h_{1}=0, \quad \triangle_{\mathrm{Z}} h_{5}=0$,

where $D_{\mu}$ is the covariant derivative constructed by the metric $q_{\mu \nu}$, and $\triangle_{\mathrm{Y}}, \triangle_{\mathrm{Z}}$ are the Laplace operators on $\mathrm{Y}, \mathrm{Z}$ space, and $R_{\mu \nu}(\mathrm{X}), R_{i j}(\mathrm{Y}), R_{a b}(\mathrm{Z})$ are the Ricci tensors associated with the metrics $q_{\mu \nu}(\mathrm{X}), \gamma_{i j}(\mathrm{Y}), u_{a b}(\mathrm{Z})$, respectively. As a special example, let us consider the case

$$
q_{\mu \nu}=\eta_{\mu \nu}, \quad \gamma_{i j}=\delta_{i j}, \quad u_{a b}=\delta_{a b},
$$

where $\eta_{\mu \nu}$ is the two-dimensional Minkowski metric and $\delta_{i j}, \delta_{a b}$ are the four-, four-dimensional Euclidean metrics, respectively. The solution for $h_{2}$ and $h_{5}$ can be obtained explicitly as

$h_{2}(x, y, z)=c_{\mu} x^{\mu}+\tilde{c}+\sum_{\ell} M_{\ell} \ln \left[\left|\boldsymbol{y}-\boldsymbol{y}_{\ell}\right|^{2}-M\left|z-z_{0}\right|\right]$,

$$
h_{5}(z)=\frac{M}{\left|z-z_{0}\right|^{2}},
$$

where $c_{\mu}, \tilde{c}, M_{\ell}$ and $M$ are constant parameters, and $\boldsymbol{y}_{\ell}$ and $z_{0}$ are constant vectors representing the positions of the branes.

\section{The intersection of two M5-branes}

Let us consider the solution of two M5-branes. We assume that the 11-dimensional metric is written by

$$
\begin{aligned}
d s^{2}= & h_{5}^{-1 / 3}(x, y, z) \bar{h}_{5}^{-1 / 3}(z)\left[q_{\mu \nu}(\mathrm{X}) d x^{\mu} d x^{\nu}\right. \\
& +h_{5}(x, y, z) \gamma_{i j}\left(\mathrm{Y}_{1}\right) d y^{i} d y^{j} \\
& +\bar{h}_{5}(z) w_{m n}\left(\mathrm{Y}_{2}\right) d v^{m} d v^{n} \\
& \left.+h_{5}(x, y, z) \bar{h}_{5}(z) u_{a b}(\mathrm{Z}) d z^{a} d z^{b}\right]
\end{aligned}
$$

where $q_{\mu \nu}$ is the four-dimensional metric which depends only on the four-dimensional coordinates $x^{\mu}, \gamma_{i j}$ is the two-dimensional metric which depends only on the two-dimensional coordinates $y^{i}, w_{m n}$ is the twodimensional metric which depends only on the twodimensional coordinates $v^{m}$, and finally $u_{a b}$ is the three-dimensional metric which depends only on the three-dimensional coordinates $z^{a}$.

We also assume that the gauge field strength $F_{(4)}$ is given by

$$
\begin{aligned}
F_{(4)}= & * d\left[h_{5}^{-1}(x, y, z) \Omega(\mathrm{X}) \wedge \Omega\left(\mathrm{Y}_{2}\right)\right. \\
& \left.+\bar{h}_{5}^{-1}(z) \Omega(\mathrm{X}) \wedge \Omega\left(\mathrm{Y}_{1}\right)\right],
\end{aligned}
$$

where $\Omega(\mathrm{X}), \Omega\left(\mathrm{Y}_{1}\right)$ and $\Omega\left(\mathrm{Y}_{2}\right)$ denote the volume 4-form, 2-form and 2-form, respectively 


$$
\begin{aligned}
\Omega(\mathrm{X}) & =\sqrt{-q} d x^{0} \wedge d x^{1} \wedge d x^{2} \wedge d x^{3} \\
\Omega\left(\mathrm{Y}_{1}\right) & =\sqrt{\gamma} d y^{1} \wedge d y^{2} \\
\Omega\left(\mathrm{Y}_{2}\right) & =\sqrt{w} d v^{1} \wedge d v^{2} .
\end{aligned}
$$

Here, $q, \gamma$ and $w$ are the determinant of the metric $q_{\mu \nu}, \gamma_{i j}$, and $w_{m n}$, respectively.

In terms of ansatz for fields (86) and (87), the field equations lead to

$$
\begin{array}{lr}
R_{\mu \nu}(\mathrm{X})=0, \quad R_{i j}\left(\mathrm{Y}_{1}\right)=0, & \\
\quad R_{m n}\left(\mathrm{Y}_{2}\right)=0, \quad R_{a b}(\mathrm{Z})=0, & (89 \mathrm{a}) \\
h_{5}=h_{0}(x)+h_{1}(y, z), & (89 \mathrm{~b}) \\
D_{\mu} D_{\nu} h_{0}=0, \quad \bar{h}_{5} \triangle_{\mathrm{Y}_{1}} h_{1}+\triangle_{\mathrm{Z}} h_{1}=0, & \triangle_{\mathrm{Z}} \bar{h}_{5}=0,
\end{array}
$$

where $D_{\mu}$ is the covariant derivative constructed by the metric $q_{\mu \nu}$, and $\triangle_{\mathrm{Y}_{1}}, \triangle_{\mathrm{Z}}$ are the Laplace operators on $\mathrm{Y}_{1}$, $\mathrm{Z}$ space, and $R_{\mu \nu}(\mathrm{X}), R_{i j}\left(\mathrm{Y}_{1}\right), R_{m n}\left(\mathrm{Y}_{2}\right), R_{a b}(\mathrm{Z})$ are the Ricci tensors associated with the metrics $q_{\mu \nu}(\mathrm{X}), \gamma_{i j}\left(\mathrm{Y}_{1}\right)$, $w_{m n}\left(\mathrm{Y}_{2}\right), u_{a b}(\mathrm{Z})$, respectively. As a special example, let us consider the case

$$
q_{\mu \nu}=\eta_{\mu \nu}, \quad \gamma_{i j}=\delta_{i j}, \quad u_{a b}=\delta_{a b},
$$

where $\eta_{\mu \nu}$ is the four-dimensional Minkowski metric and $\delta_{i j}, \delta_{m n}, \delta_{a b}$ are the two-, two-, three-dimensional Euclidean metrics, respectively. The solution for $h_{5}$ and $\bar{h}_{5}$ can be obtained explicitly as

$$
\begin{aligned}
h_{2}(x, y, z) & =c_{\mu} x^{\mu}+\tilde{c}+\sum_{\ell} \frac{M_{\ell}}{\left[\left|\boldsymbol{y}-\boldsymbol{y}_{\ell}\right|^{2}+4 M\left|z-z_{0}\right|\right]^{2}}, \\
h_{5}(z) & =\frac{M}{\left|z-z_{0}\right|},
\end{aligned}
$$

where $c_{\mu}, \tilde{c}, M_{\ell}$ and $M$ are constant parameters, and $\boldsymbol{y}_{\ell}$ and $z_{0}$ are constant vectors representing the positions of the branes.

\section{The intersection of M2-brane and one Kaluza-Klein monopole}

Now we discuss the KK-monopole in the transverse space of M2-brane. We assume that the 11-dimensional metric takes the form

$$
\begin{aligned}
d s^{2}= & h_{2}^{-2 / 3}(x, y, z) q_{\mu \nu}(\mathrm{X}) d x^{\mu} d x^{\nu} \\
& +h_{2}^{1 / 3}(x, y, z)\left[\gamma_{i j}(\mathrm{Y}) d y^{i} d y^{j}+h_{k}(z) u_{a b}(\mathrm{Z}) d z^{a} d z^{b}\right. \\
& \left.+h_{k}^{-1}(z)\left(d v+A_{a} d z^{a}\right)^{2}\right]
\end{aligned}
$$

where $q_{\mu \nu}$ is the three-dimensional metric which depends only on the three-dimensional coordinates $x^{\mu}, \gamma_{i j}$ is the four-dimensional metric which depends only on the four-dimensional coordinates $y^{i}$, and finally $u_{a b}$ is the three-dimensional metric which depends only on the threedimensional coordinates $z^{a}$.

We also assume that the gauge field strength $F_{(4)}$ is given by

$$
F_{(4)}=d\left[h_{2}^{-1}(x, y, z)\right] \wedge \Omega(\mathrm{X}),
$$

where $\Omega(\mathrm{X})$ denotes the volume3-form

$$
\Omega(\mathrm{X})=\sqrt{-q} d x^{0} \wedge d x^{1} \wedge d x^{2} .
$$

Here, $q$ is the determinant of the metric $q_{\mu \nu}$.

In terms of ansatz for fields (92) and (93), the field equations lead to

$R_{\mu \nu}(\mathrm{X})=0, \quad R_{i j}(\mathrm{Y})=0, \quad R_{a b}(\mathrm{Z})=0$,

$h_{2}=h_{0}(x)+h_{1}(y, z), \quad d h_{k}=*_{\mathrm{Z}} d A$,

$D_{\mu} D_{\nu} h_{0}=0, \quad h_{k} \triangle_{\mathrm{Y}} h_{1}+\triangle_{\mathrm{Z}} h_{1}=0, \quad \triangle_{\mathrm{Z}} h_{k}=0$,

where $D_{\mu}$ is the covariant derivative with respect to the metric $q_{\mu \nu}$, and $\triangle_{\mathrm{Y}}, \triangle_{\mathrm{Z}}$ are the Laplace operators on $\mathrm{X}, \mathrm{Y}, \mathrm{Z}$ space, and $R_{\mu \nu}(\mathrm{X}), R_{i j}(\mathrm{Y}), R_{a b}(\mathrm{Z})$ are the Ricci tensors associated with the metrics $q_{\mu \nu}(\mathrm{X}), \gamma_{i j}(\mathrm{Y}), u_{a b}(\mathrm{Z})$, respectively.

As a special example, let us consider the case

$$
q_{\mu \nu}=\eta_{\mu \nu}, \quad \gamma_{i j}=\delta_{i j}, \quad u_{a b}=\delta_{a b},
$$

where $\eta_{\mu \nu}$ is the three-dimensional Minkowski metric and $\delta_{i j}, \delta_{a b}$ are the four-, three-dimensional Euclidean metrics, respectively. The solution for $h_{2}$ and $h_{k}$ can be obtained explicitly as

$$
\begin{aligned}
h_{2}(x, y, z) & =c_{\mu} x^{\mu}+\tilde{c}+\sum_{\ell} \frac{M_{\ell}}{\left[\left|\boldsymbol{y}-\boldsymbol{y}_{\ell}\right|^{2}+4 M\left|z-z_{0}\right|\right]^{3}}, \\
h_{k}(z) & =\frac{M}{\left|z-z_{0}\right|},
\end{aligned}
$$

where $c_{\mu}, \tilde{c}, M_{\ell}$ and $M$ are constant parameters, and $\boldsymbol{y}_{\ell}$ and $z_{0}$ are constant vectors representing the positions of the branes. Since the functions coincide, the locations of the branes will also coincide.

\section{E. The intersection of M5-brane and one Kaluza-Klein monopole}

In this subsection, we discuss the KK-monopole in the transverse space of the M5-brane. We assume that the 11-dimensional metric takes the form

$$
\begin{aligned}
d s^{2}= & h_{5}^{-1 / 3}(x, y, z) q_{\mu \nu}(\mathrm{X}) d x^{\mu} d x^{\nu}+h_{5}^{2 / 3}(x, y, z) \\
& \times\left[d y^{2}+h_{k}(z) u_{a b}(\mathrm{Z}) d z^{a} d z^{b}+h_{k}^{-1}(z)\left(d v+A_{a} d z^{a}\right)^{2}\right],
\end{aligned}
$$

where $q_{\mu \nu}$ is the six-dimensional metric which depends only on the six-dimensional coordinates $x^{\mu}$, and finally $u_{a b}$ 
is the three-dimensional metric which depends only on the three-dimensional coordinates $z^{a}$.

We also assume that the gauge field strength $F_{(4)}$ is given by

$$
F_{(4)}=* d\left[h_{5}^{-1}(x, y, z) \wedge \Omega(\mathrm{X})\right],
$$

where $\Omega(\mathrm{X})$ denotes the volume 6-form

$$
\Omega(\mathrm{X})=\sqrt{-q} d x^{0} \wedge d x^{1} \wedge \cdots \wedge d x^{5} .
$$

Here, $q$ is the determinant of the metric $q_{\mu \nu}$.

In terms of ansatz for fields (98) and (99), the field equations lead to

$R_{\mu \nu}(\mathrm{X})=0, \quad R_{a b}(\mathrm{Z})=0$,

$h_{5}=h_{0}(x)+h_{1}(y, z), \quad d h_{k}=*_{\mathrm{Z}} d A$,

$D_{\mu} D_{\nu} h_{0}=0, \quad h_{k} \partial_{y}^{2} h_{1}+\triangle_{\mathrm{Z}} h_{1}=0, \quad \triangle_{\mathrm{Z}} h_{k}=0$,

where $D_{\mu}$ is the covariant derivative with respect to the metric $q_{\mu \nu}$, and $\triangle_{\mathrm{X}}, \triangle_{\mathrm{Z}}$ are the Laplace operators on $\mathrm{X}, \mathrm{Z}$ space, and $R_{\mu \nu}(\mathrm{X}), R_{a b}(\mathrm{Z})$ are the Ricci tensors associated with the metrics $q_{\mu \nu}(\mathrm{X}), u_{a b}(\mathrm{Z})$, respectively.

As a special example, let us consider the case

$$
q_{\mu \nu}=\eta_{\mu \nu}, \quad u_{a b}=\delta_{a b},
$$

where $\eta_{\mu \nu}$ is the six-dimensional Minkowski metric and $\delta_{a b}$ is the three-dimensional Euclidean metric. The solution for $h_{5}$ and $h_{k}$ can be obtained explicitly as

$$
\begin{aligned}
h_{5}(x, y, z) & =c_{\mu} x^{\mu}+\tilde{c}+\sum_{\ell} \frac{M_{\ell}}{\left[\left|\boldsymbol{y}-\boldsymbol{y}_{\ell}\right|^{2}+4 M\left|z-z_{0}\right|\right]^{3 / 2}}, \\
h_{k}(z) & =\frac{M}{\left|z-z_{0}\right|},
\end{aligned}
$$

where $c_{\mu}, \tilde{c}, M_{\ell}$ and $M$ are constant parameters, and $\boldsymbol{y}_{\ell}$ and $z_{0}$ are constant vectors representing the positions of the branes. Since the functions coincide, the locations of the branes will also coincide.

\section{F. The intersection involving plane wave and M2-brane}

We present the M2-brane with the plane wave propagating along its longitudinal direction. We assume that the 11dimensional metric takes the form

$$
\begin{aligned}
d s^{2}= & h_{2}^{-2 / 3}(z)\left[-d t^{2}+d x^{2}+d y^{2}+\left\{h_{w}(t, y, z)-1\right\}\right. \\
& \left.\times(d t-d x)^{2}+h_{2}(z) u_{a b}(\mathrm{Z}) d z^{a} d z^{b}\right],
\end{aligned}
$$

where $u_{a b}$ is the eight-dimensional metric which depends only on the eight-dimensional coordinates $z^{a}$.

We also assume that the gauge field strength $F_{(4)}$ is given by

$$
F_{(4)}=d\left[h_{2}^{-1}(z)\right] \wedge d t \wedge d x \wedge d y .
$$

In terms of ansatz for fields (104) and (105), the field equations lead to

$$
\begin{aligned}
& R_{a b}(\mathrm{Z})=0, \\
& h_{w}=h_{0}(t)+h_{1}(y, z),
\end{aligned}
$$

where $\triangle_{\mathrm{Z}}$ is the Laplace operator on $\mathrm{Z}$ space, and $R_{a b}(\mathrm{Z})$ are the Ricci tensor associated with the metric $u_{a b}(\mathrm{Z})$. As a special example, let us consider the case

$$
u_{a b}=\delta_{a b},
$$

where $\delta_{a b}$ is the eight-dimensional Euclidean metrics, respectively. The solution for $h_{2}$ and $h_{w}$ can be obtained explicitly as

$$
\begin{aligned}
h_{w}(t, y, z) & =\bar{c} t+\tilde{c}+\sum_{\ell} \frac{M_{\ell}}{\left[\left|y-y_{\ell}\right|^{2}+\frac{M}{4}\left|z-z_{0}\right|^{-4}\right]^{-1}}, \\
h_{2}(z) & =\frac{M}{\left|z-z_{0}\right|^{6}},
\end{aligned}
$$

where $\bar{c}, \tilde{c}, M_{\ell}$ and $M$ are constant parameters, and $y_{\ell}$ and $z_{0}$ are constant vectors representing the positions of the branes. If we delocalize along $n$ of the overall transverse directions, the harmonic functions take the following form:

$$
\begin{aligned}
h_{w}(t, y, z)= & \bar{c} t+\tilde{c} \\
& +\sum_{\ell} \frac{M_{\ell}}{\left[\left|y-y_{\ell}\right|^{2}+\frac{4 M}{(n-4)^{2}}\left|z-z_{0}\right|^{n-4}\right]^{8-n / 2(n-4)}}, \\
h_{2}(z)= & \frac{M}{\left|z-z_{0}\right|^{6-n}} .
\end{aligned}
$$

\section{G. The intersection involving wave and M5-brane}

We present the M5-brane with the plane wave propagating along its longitudinal direction. We assume that the 11dimensional metric takes the form

$$
\begin{aligned}
d s^{2}= & h_{5}^{-1 / 3}(z)\left[-d t^{2}+d x^{2}+\left\{h_{w}(t, y, z)-1\right\}(d t-d x)^{2}\right. \\
& \left.+\gamma_{i j}(\mathrm{Y}) d y^{i} d y^{j}+h_{5}(z) u_{a b}(\mathrm{Z}) d z^{a} d z^{b}\right],
\end{aligned}
$$

where $\gamma_{i j}$ is the four-dimensional metric which depends only on the four-dimensional coordinates $y^{i}$, and finally $u_{a b}$ is the five-dimensional metric which depends only on the five-dimensional coordinates $z^{a}$.

We also assume that the gauge field strength $F_{(4)}$ is given by

$$
F_{(4)}=* d\left[h_{5}^{-1}(z) \wedge d t \wedge d x \wedge \Omega(\mathrm{Y})\right],
$$

where $\Omega(\mathrm{Y})$ denotes the volume 4-form 
DYNAMICS OF PARTIALLY LOCALIZED BRANE SYSTEMS

PHYSICAL REVIEW D 84, 126006 (2011)

$$
\Omega(\mathrm{Y})=\sqrt{\gamma} d y^{1} \wedge d y^{2} \wedge d y^{3} \wedge d y^{4} .
$$

Here, $\gamma$ is the determinant of the metric $\gamma_{i j}$.

In terms of ansatz for fields (110) and (111), the field equations lead to

$$
\begin{aligned}
& R_{i j}(\mathrm{Y})=0, \quad R_{a b}(\mathrm{Z})=0, \\
& h_{w}=h_{0}(t)+h_{1}(y, z), \quad \partial_{t}^{2} h_{0}=0, \\
& \quad h_{5} \triangle_{\mathrm{Y}} h_{1}+\triangle_{\mathrm{Z}} h_{1}=0, \quad \triangle_{\mathrm{Z}} h_{5}=0,
\end{aligned}
$$

where $\triangle_{\mathrm{Y}}, \triangle_{\mathrm{Z}}$ are the Laplace operators on $\mathrm{Y}, \mathrm{Z}$ space, and $R_{i j}(\mathrm{Y}), R_{a b}(\mathrm{Z})$ are the Ricci tensors associated with the metrics $\gamma_{i j}(\mathrm{Y}), u_{a b}(\mathrm{Z})$, respectively. As a special example, let us consider the case

$$
\gamma_{i j}=\delta_{i j}, \quad u_{a b}=\delta_{a b},
$$

where $\delta_{i j}, \delta_{a b}$ are the four-dimensional Euclidean metrics, respectively. The solution for $h_{5}$ and $h_{w}$ can be obtained explicitly as

$$
\begin{aligned}
h_{w}(t, y, z) & =\bar{c} t+\tilde{c}+\sum_{\ell} \frac{M_{\ell}}{\left[\left|\boldsymbol{y}-\boldsymbol{y}_{\ell}\right|^{2}+4 M\left|z-z_{0}\right|^{-1}\right]^{-1}}, \\
h_{5}(z) & =\frac{M}{\left|z-z_{0}\right|^{3}},
\end{aligned}
$$

where $\bar{c}, \tilde{c}, M_{\ell}$ and $M$ are constant parameters, and $\boldsymbol{y}_{\ell}$ and $z_{0}$ are constant vectors representing the positions of the branes. If we delocalize along $n$ of the overall transverse directions, the harmonic functions take the following form:

$$
\begin{aligned}
h_{w}(t, y, z)= & \bar{c} t+\tilde{c} \\
& +\sum_{\ell} \frac{M_{\ell}}{\left[\left|\boldsymbol{y}-\boldsymbol{y}_{\ell}\right|^{2}+\frac{4 M}{(n-1)^{2}}\left|z-z_{0}\right|^{n-1}\right]^{(n+1) /(n-1)}}, \\
h_{5}(z)= & \frac{M}{\left|z-z_{0}\right|^{3-n}} .
\end{aligned}
$$

\section{H. The plane wave in the KK-monopole background}

We consider the plane wave propagating in the background of the KK-monopole. The solution of tendimensional metric is given by

$$
\begin{aligned}
d s^{2}= & -d t^{2}+d x^{2}+\left\{h_{w}(t, y, z)-1\right\}(d t-d x)^{2} \\
& +\gamma_{i j}(\mathrm{Y}) d y^{i} d y^{j}+h_{k}(z) u_{a b}(\mathrm{Z}) d z^{a} d z^{b} \\
& +h_{k}^{-1}(z)\left(d v+A_{a} d z^{a}\right)^{2},
\end{aligned}
$$

where $\gamma_{i j}$ is the five-dimensional metric which depends only on the five-dimensional coordinates $y^{i}$, and finally $u_{a b}$ is the three-dimensional metric which depends only on the three-dimensional coordinates $z^{a}$.

The ten-dimensional metric and the function $h_{k}$ obey

$$
\begin{aligned}
& R_{i j}(\mathrm{Y})=0, \quad R_{a b}(\mathrm{Z})=0, \\
& h_{w}=h_{0}(t)+h_{1}(y, z), \quad \partial_{t}^{2} h_{0}=0, \\
& \quad h_{k} \triangle_{\mathrm{Y}} h_{1}+\triangle_{\mathrm{Z}} h_{1}=0, \quad \triangle_{\mathrm{Z}} h_{k}=0, \\
& d h_{k}=*_{\mathrm{Z}} d A,
\end{aligned}
$$

where $*_{\mathrm{Z}}$ is the Hodge operator in the $\mathrm{Z}$ space, and $\triangle_{\mathrm{Y}}, \Delta_{\mathrm{Z}}$ are the Laplace operators on $\mathrm{Y}, \mathrm{Z}$ space, and $R_{i j}(\mathrm{Y}), R_{a b}(\mathrm{Z})$ are the Ricci tensors associated with the metrics $q_{\mu \nu}(\mathrm{X})$, $\gamma_{i j}(\mathrm{Y}), u_{a b}(\mathrm{Z})$, respectively. As a special example, let us consider the case

$$
\gamma_{i j}=\delta_{i j}, \quad u_{a b}=\delta_{a b},
$$

where $\delta_{i j}, \delta_{a b}$ are the five-, three-dimensional Euclidean metrics, respectively. The solution for $h$ and $h_{k}$ can be obtained explicitly as

$$
\begin{aligned}
h_{w}(t, y, z) & =\bar{c} t+\tilde{c}+\sum_{\ell} \frac{M_{\ell}}{\left[\left|\boldsymbol{y}-\boldsymbol{y}_{\ell}\right|^{2}+4 M\left|z-z_{0}\right|\right]^{7 / 2}}, \\
h_{k}(z) & =\frac{M}{\left|z-z_{0}\right|},
\end{aligned}
$$

where $\bar{c}, \tilde{c}, M_{\ell}$ and $M$ are constant parameters, and $\boldsymbol{y}_{\ell}$ and $z_{0}$ are constant vectors representing the positions of the

TABLE IV. Pair intersections between M-brane and KK-monopole in $D=11$ with dependence on overall transverse coordinates.

\begin{tabular}{lcccccccccccccccc}
\hline \hline Case & & 0 & 1 & 2 & 3 & 4 & 5 & 6 & 7 & 8 & 9 & 10 & $\tilde{\mathrm{M}}$ & $\lambda(\tilde{\mathrm{M}})$ & $\lambda_{\mathrm{E}}(\tilde{\mathrm{M}})$ \\
\hline & $\mathrm{M} 2$ & $\circ$ & $\circ$ & $\circ$ & & & & & & & & $\sqrt{ }$ & & $\lambda(\mathrm{Y})=2 / 5$ & $\lambda_{\mathrm{E}}(\mathrm{Y})=\frac{-3+d_{1}}{-12+2 d_{1}+d_{2}+d_{3}}$ \\
$\mathrm{M} 2-\mathrm{KK}$ & $\mathrm{KK}$ & $\circ$ & $\circ$ & $\circ$ & $\circ$ & $\circ$ & $\circ$ & $\circ$ & & $A_{1}$ & $A_{2}$ & $A_{3}$ & $\mathrm{Y} \& v \& \mathrm{Z}$ & $\lambda(v)=2 / 5$ & $\lambda_{\mathrm{E}}(v)=\frac{-3+d_{1}}{-12+2 d_{1}+d_{2}+d_{3}}$ \\
& $x^{N}$ & $t$ & $x^{1}$ & $x^{2}$ & $y^{1}$ & $y^{2}$ & $y^{3}$ & $y^{4}$ & $v$ & $z^{1}$ & $z^{2}$ & $z^{3}$ & & & $\lambda(\mathrm{Z})=2 / 5$ & $\lambda_{\mathrm{E}}(\mathrm{Z})=\frac{-3+d_{1}}{-12+2 d_{1}+d_{2}+d_{3}}$ \\
& $\mathrm{M} 5$ & $\circ$ & $\circ$ & $\circ$ & $\circ$ & $\circ$ & $\circ$ & & & & & & $\sqrt{ }$ & $\tilde{\mathrm{X}}$ & $\lambda(\tilde{\mathrm{X}})=-1 / 5$ & $\lambda_{\mathrm{E}}(\tilde{\mathrm{X}})=\frac{3-d_{2}-d_{3}-d_{4}}{-15+2 d_{1}+d_{2}+d_{3}}$ \\
$\mathrm{M} 5-\mathrm{KK}$ & $\mathrm{KK}$ & $\circ$ & $\circ$ & $\circ$ & $\circ$ & $\circ$ & $\circ$ & $\circ$ & & $A_{1}$ & $A_{2}$ & $A_{3}$ & $\mathrm{Y} \& v \& \mathrm{Z}$ & $\lambda(\mathrm{Y})=2 / 5$ & $\lambda_{\mathrm{E}}(\mathrm{Y})=\frac{6-d_{1}}{15-2 d_{1}-d_{2}-d_{3}}$ \\
& $x^{N}$ & $t$ & $x^{1}$ & $x^{2}$ & $x^{3}$ & $x^{4}$ & $x^{5}$ & $y$ & $v$ & $z^{1}$ & $z^{2}$ & $z^{3}$ & & & $\lambda(v)=\lambda(\mathrm{Z})=2 / 5$ & $\lambda_{\mathrm{E}}(v)=\lambda_{\mathrm{E}}(\mathrm{Z})=\frac{6-d_{1}}{15-2 d_{1}-d_{2}-d_{3}}$ \\
\hline \hline
\end{tabular}


branes. Since the functions coincide, the locations of the branes will also coincide.

For the static case, there is a classification of the multiple intersecting branes with the M-waves and/or KKmonopoles [27,28]. The dynamical delocalized branes are also classified in [2]. We show the intersection rule for the branes with M-wave and KK-monopoles, which is summarized in Table IV. In the Table, circles indicate where the brane world-volumes enter, $v$ represents the coordinate of the KK-monopole, and the time-dependent branes are indicated by $\sqrt{ }$ for different solutions.

\section{THE INTERSECTION OF DYNAMICAL BRANES IN TEN-DIMENSIONAL THEORY}

In this section, we apply the dynamical brane solutions to ten-dimensional string theory. The ten-dimensional action for the $p$-brane system in the Einstein frame can be written as

$$
\begin{aligned}
S= & \frac{1}{2 \kappa^{2}} \int\left[R * \mathbf{1}-\frac{1}{2} d \phi \wedge * d \phi-\frac{1}{2 \cdot 3 !} \mathrm{e}^{-\phi} H_{(3)} \wedge * H_{(3)}\right. \\
& \left.-\sum_{I} \frac{1}{2 \cdot\left(p_{I}+2\right) !} \mathrm{e}^{\left(3-p_{I}\right) \phi / 2} F_{\left(p_{I}+2\right)} \wedge * F_{\left(p_{I}+2\right)}\right]
\end{aligned}
$$

where $\kappa^{2}$ is the ten-dimensional gravitational constant, and $\phi$ is the dilaton, and $*$ is the Hodge operator in the tendimensional spacetime, and $H_{(3)}, F_{\left(p_{I}+2\right)}$ are 3-, $\left(p_{I}+\right.$ 2)-form field strength, respectively. We assume that the field strengths $H_{(3)}, F_{\left(p_{I}+2\right)}$ are given by following gauge potentials

$$
H_{(3)}=d B_{(2)}, \quad F_{\left(p_{I}+2\right)}=d C_{\left(p_{I}+1\right)} .
$$

The field equations are given by

$$
\begin{aligned}
& R_{M N}=\frac{1}{2} \partial_{M} \phi \partial_{N} \phi+\frac{1}{2 \cdot 3 !} \mathrm{e}^{-\phi}\left[3 F_{M A B} H_{N}^{A B}-\frac{1}{4} g_{M N} H_{(3)}^{2}\right] \\
& \quad+\sum_{I} \frac{\mathrm{e}^{\left(3-p_{I}\right) \phi / 2}}{2 \cdot\left(p_{I}+2\right) !}\left[\left(p_{I}+2\right) F_{M A_{1} \cdots A_{p_{I}+1}} H_{N}^{A_{1} \cdots A_{p_{I}+1}}-\frac{1}{8}\left(p_{I}+1\right) g_{M N} F_{\left(p_{I}+2\right)}^{2}\right], \\
& d * d \phi+\frac{1}{2 \cdot 3 !} \mathrm{e}^{-\phi} H_{(3)} \wedge * H_{(3)}-\sum_{I} \frac{\left(3-p_{I}\right)}{4 \cdot\left(p_{I}+2\right) !} \mathrm{e}^{\left(3-p_{I}\right) \phi / 2} F_{\left(p_{I}+2\right)} \wedge * F_{\left(p_{I}+2\right)}=0, \\
& d\left[\mathrm{e}^{-\phi} * H_{(3)}\right]=0, \\
& d\left[\mathrm{e}^{\left(3-p_{I}\right) \phi / 2} * F_{\left(p_{I}+2\right)}\right]=0 .
\end{aligned}
$$

In what follows, we look for the possible configurations of intersecting branes and present explicit solutions. The case with waves or KK-monopoles will be also discussed.

\section{A. The intersection involving two D $p$-brane}

Let us first discuss the dynamical solution of two $\mathrm{D} p$-branes. The ten-dimensional metric thus takes the form

$$
\begin{aligned}
d s^{2}= & h^{(p-7) / 8}(x, y, z) \bar{h}^{(p-7) / 8}(z)\left[q_{\mu \nu}(\mathrm{X}) d x^{\mu} d x^{\nu}\right. \\
& +h(x, y, z) \gamma_{i j}\left(\mathrm{Y}_{1}\right) d y^{i} d y^{j}+\bar{h}(z) w_{m n}\left(\mathrm{Y}_{2}\right) d v^{m} d v^{n} \\
& \left.+h(x, y, z) \bar{h}(z) u_{a b}(\mathrm{Z}) d z^{a} d z^{b}\right],
\end{aligned}
$$

where $q_{\mu \nu}$ is the $(p-1)$-dimensional metric which depends only on the $(p-1)$-dimensional coordinates $x^{\mu}, \gamma_{i j}$ is the two-dimensional metric which depends only on the two-dimensional coordinates $y^{i}, w_{m n}$ is the twodimensional metric which depends only on the twodimensional coordinates $w^{m}$, and finally $u_{a b}$ is the $(7-p)$-dimensional metric which depends only on the $(7-p)$-dimensional coordinates $z^{a}$.

We also assume that the scalar field $\phi$ and the gauge field strength $F_{(4)}$ are given by

$$
\begin{aligned}
\mathrm{e}^{\phi}= & (h \bar{h})^{(3-p) / 4}, \\
F_{(p+2)}= & d\left[h^{-1}(x, y, z)\right] \Omega(\mathrm{X}) \wedge \Omega\left(\mathrm{Y}_{1}\right) \\
& +d\left[\bar{h}^{-1}(z)\right] \Omega(\mathrm{X}) \wedge \Omega\left(\mathrm{Y}_{2}\right),
\end{aligned}
$$

where $\Omega(\mathrm{X}), \Omega\left(\mathrm{Y}_{1}\right), \Omega\left(\mathrm{Y}_{2}\right)$ denote the volume ( $\left.p-1\right)$-, 2-, 2-form, respectively

$$
\begin{aligned}
\Omega(\mathrm{X}) & =\sqrt{-q} d x^{0} \wedge d x^{1} \wedge \cdots \wedge d x^{p-2} \\
\Omega\left(\mathrm{Y}_{1}\right) & =\sqrt{\gamma} d y^{1} \wedge d y^{2} \\
\Omega\left(\mathrm{Y}_{2}\right) & =\sqrt{w} d v^{1} \wedge d v^{2}
\end{aligned}
$$

Here, $q, \gamma, w$ are the determinant of the metrics $q_{\mu \nu}, \gamma_{i j}$, $w_{m n}$.

In terms of ansatz for fields (124) and (125), the field equations lead to

$$
\begin{aligned}
& R_{\mu \nu}(\mathrm{X})=0, \quad R_{i j}\left(\mathrm{Y}_{1}\right)=0, \\
& R_{m n}\left(\mathrm{Y}_{2}\right)=0, \quad R_{a b}(\mathrm{Z})=0, \\
& h=h_{0}(x)+h_{1}(y, z) \quad D_{\mu} D_{\nu} h_{0}=0, \\
& \bar{h} \triangle_{\mathrm{Y}_{1}} h_{1}+\triangle_{\mathrm{Z}} h_{1}=0, \quad \triangle_{\mathrm{Z}} \bar{h}=0,
\end{aligned}
$$

where $D_{\mu}$ is the covariant derivative with respect to the metric $q_{\mu \nu}$, and $\triangle_{\mathrm{Y}_{1}}, \triangle_{\mathrm{Z}}$ are the Laplace operators on $\mathrm{Y}_{1}$, $\mathrm{Z}$ space, and $R_{\mu \nu}(\mathrm{X}), R_{i j}\left(\mathrm{Y}_{1}\right), R_{m n}\left(\mathrm{Y}_{2}\right), R_{a b}(\mathrm{Z})$ are the 


\section{DYNAMICS OF PARTIALLY LOCALIZED BRANE SYSTEMS}

Ricci tensors associated with the metrics $q_{\mu \nu}(\mathrm{X}), \gamma_{i j}\left(\mathrm{Y}_{1}\right)$, $w_{m n}\left(\mathrm{Y}_{2}\right), u_{a b}(\mathrm{Z})$, respectively.

Let us consider the case

$$
q_{\mu \nu}=\eta_{\mu \nu}, \quad \gamma_{i j}=\delta_{i j}, \quad w_{m n}=\delta_{m n}, \quad u_{a b}=\delta_{a b},
$$

where $\eta_{\mu \nu}$ is the $(p-1)$-dimensional Minkowski metric and $\delta_{i j}, \delta_{m n}, \delta_{a b}$ are the two-, two-, $(7-p)$-dimensional Euclidean metrics, respectively. For $p \neq 3$ and $p \neq 5$, the solution for $h$ and $\bar{h}$ can be obtained explicitly as

$$
\begin{aligned}
h(x, y, z)= & c_{\mu} x^{\mu}+\tilde{c} \\
& +\sum_{\ell} \frac{M_{\ell}}{\left[\left|\boldsymbol{y}-\boldsymbol{y}_{\ell}\right|^{2}+\frac{4 M}{(p-3)^{2}}\left|z-z_{0}\right|^{p-3}\right]^{2 /(p-3)}}, \\
\bar{h}(z)= & \frac{M}{\left|z-z_{0}\right|^{5-p}},
\end{aligned}
$$

where $c_{\mu}, \tilde{c}, M_{\ell}$ and $M$ are constant parameters, and $\boldsymbol{y}_{\ell}$ and $z_{0}$ are constant vectors representing the positions of the branes. Since the functions coincide, the locations of the branes will also coincide. In the case of $p=3$, the field equations give

$$
h_{\mathrm{F}}(x, y, z)=c_{\mu} x^{\mu}+\tilde{c}+\sum_{\ell} M_{\ell} \ln \left[\left|\boldsymbol{y}-\boldsymbol{y}_{\ell}\right|^{2}-2 M\left|z-z_{0}\right|\right] \text {, }
$$

$$
h(z)=\frac{M}{\left|z-z_{0}\right|^{2}} .
$$

For $p=5$, the solution becomes

$$
\begin{aligned}
h(x, y, z) & =c_{\mu} x^{\mu}+\tilde{c}+\sum_{\ell} \frac{M_{\ell}}{\left[\left|\boldsymbol{y}-\boldsymbol{y}_{\ell}\right|^{2}+M\left|z-z_{0}\right|^{2}\right]}, \\
\bar{h}(z) & =M \ln \left|z-z_{0}\right| .
\end{aligned}
$$

\section{B. The intersection of $\mathrm{D} p-\mathrm{D}(\boldsymbol{p}+2)$ branes}

Next we discuss the $\mathrm{D} p$-branes ending on $\mathrm{D}(p+$ 2)-branes. Let us consider the solution to be delocalized along the relative transverse direction of the $\mathrm{D} p$-branes. The ten-dimensional metric thus takes the form

$$
\begin{aligned}
d s^{2}= & h_{p}^{(p-7) / 8}(x, y, z) h_{p+2}^{(p-5) / 8}(z)\left[q_{\mu \nu}(\mathrm{X}) d x^{\mu} d x^{\nu}\right. \\
& +h_{p}(x, y, z) \gamma_{i j}(\mathrm{Y}) d y^{i} d y^{j}+h_{p+2}(z) d v^{2} \\
& \left.+h_{p}(x, y, z) h_{p+2}(z) u_{a b}(\mathrm{Z}) d z^{a} d z^{b}\right]
\end{aligned}
$$

where $q_{\mu \nu}$ is the $p$-dimensional metric which depends only on the $p$-dimensional coordinates $x^{\mu}, \gamma_{i j}$ is the threedimensional metric which depends only on the threedimensional coordinates $y^{i}$, and finally $u_{a b}$ is the
PHYSICAL REVIEW D 84, 126006 (2011)

$(6-p)$-dimensional metric which depends only on the $(6-p)$-dimensional coordinates $z^{a}$.

We also assume that the scalar field $\phi$ and the gauge field strengths $F_{(p+2)}, F_{(p+4)}$ are given by

$$
\begin{aligned}
\mathrm{e}^{\phi} & =h_{p}^{(3-p) / 4} h_{p+2}^{(1-p) / 4}, \\
F_{(p+2)} & =d\left[h_{p}^{-1}(x, y, z)\right] \wedge \Omega(\mathrm{X}) \wedge d v, \\
F_{(p+4)} & =d\left[h_{p+2}^{-1}(z)\right] \wedge \Omega(\mathrm{X}) \wedge \Omega(\mathrm{Y}),
\end{aligned}
$$

where $\Omega(\mathrm{X})$ and $\Omega(\mathrm{Y})$ denote the volume $p$-, 3-form, respectively

$$
\begin{aligned}
& \Omega(\mathrm{X})=\sqrt{-q} d x^{0} \wedge d x^{1} \wedge \cdots \wedge d x^{p-1} \\
& \Omega(\mathrm{Y})=\sqrt{\gamma} d y^{1} \wedge d y^{2} \wedge d y^{3} .
\end{aligned}
$$

Here, $q, \gamma$ are the determinant of the metrics $q_{\mu \nu}, \gamma_{i j}$.

Under the assumptions (162) and (163), the field equations lead to

$$
\begin{aligned}
& R_{\mu \nu}(\mathrm{X})=0, \quad R_{i j}(\mathrm{Y})=0, \quad R_{a b}(\mathrm{Z})=0, \\
& h_{p}=h_{0}(x)+h_{1}(y, z), \quad D_{\mu} D_{\nu} h_{0}=0, \\
& h_{p+2} \triangle_{\mathrm{Y}} h_{1}+\triangle_{\mathrm{Z}} h_{1}=0, \quad \triangle_{\mathrm{Z}} h_{p+2}=0,
\end{aligned}
$$

where $D_{\mu}$ is the covariant derivative with respect to the metric $q_{\mu \nu}$, and $\triangle_{\mathrm{Y}}, \triangle_{\mathrm{Z}}$ are the Laplace operators on $\mathrm{Y}, \mathrm{Z}$ space, and $R_{i j}(\mathrm{X}), R_{i j}(\mathrm{Y}), R_{a b}(\mathrm{Z})$ are the Ricci tensors associated with the metrics $q_{\mu \nu}(\mathrm{X}), \gamma_{i j}(\mathrm{Y}), u_{a b}(\mathrm{Z})$, respectively. Now we assume that the ten-dimensional metric is given by

$$
q_{\mu \nu}=\eta_{\mu \nu}, \quad \gamma_{i j}=\delta_{i j}, \quad u_{a b}=\delta_{a b},
$$

where $\eta_{\mu \nu}$ is the $p$-dimensional Minkowski metric and $\delta_{i j}$, $\delta_{a b}$ are the three-, $(6-p)$-dimensional Euclidean metrics, respectively. For $p \neq 2, p \neq 4$, the solution for $h_{p}$ and $h_{p+2}$ can be obtained explicitly as

$$
\begin{aligned}
h_{p}(x, y, z)= & c_{\mu} x^{\mu}+\tilde{c} \\
& +\sum_{\ell} \frac{M_{\ell}}{\left[\left|\boldsymbol{y}-\boldsymbol{y}_{\ell}\right|^{2}+\frac{4 M}{(p-2)^{2}}\left|z-z_{0}\right|^{p-2}\right]^{p+2 / 2(p-2)}}, \\
h_{p+2}(z)= & \frac{M}{\left|z-z_{0}\right|^{4-p}},
\end{aligned}
$$

where $A, \tilde{c}, M_{\ell}$ and $M$ are constant parameters, and $\boldsymbol{y}_{\ell}$ and $z_{0}$ are constant vectors representing the positions of the branes.

If we set $p=2$, the solution becomes

$$
\begin{aligned}
h_{2}(x, y, z)= & c_{\mu} x^{\mu}+\tilde{c} \\
& +\sum_{\ell} M_{\ell} \ln \left[\left|\boldsymbol{y}-\boldsymbol{y}_{\ell}\right|^{2}-3 M\left|z-z_{0}\right|\right], \\
h_{4}(z)= & \frac{M}{\left|z-z_{0}\right|^{2}} .
\end{aligned}
$$


MASATO MINAMITSUJI AND KUNIHITO UZAWA

Next we consider the case of $p=4$. The solution is given by

$$
\begin{aligned}
h_{4}(x, y, z) & =c_{\mu} x^{\mu}+\tilde{c}+\sum_{\ell} \frac{M_{\ell}}{\left[\left|\boldsymbol{y}-\boldsymbol{y}_{\ell}\right|^{2}+M\left|z-z_{0}\right|^{2}\right]^{(3 / 2)}}, \\
h_{6}(z) & =M \ln \left|z-z_{0}\right| .
\end{aligned}
$$

\section{The $\mathrm{D} \boldsymbol{p}-\mathrm{D}(\boldsymbol{p}+\mathbf{4})$ brane system}

Now we consider the dynamical solution of $\mathrm{D} p-$ $\mathrm{D}(p+4)$ brane system. Let us discuss the solution to be delocalized along the relative transverse direction of the $\mathrm{D} p$-branes. The ten-dimensional metric thus takes the form

$$
\begin{aligned}
d s^{2}= & h_{p}^{(p-7) / 8}(x, y, z) h_{p+4}^{(p-3) / 8}(z)\left[q_{\mu \nu}(\mathrm{X}) d x^{\mu} d x^{\nu}\right. \\
& +h_{p}(x, y, z) \gamma_{i j}(\mathrm{Y}) d y^{i} d y^{j} \\
& \left.+h_{p}(x, y, z) h_{p+4}(z) u_{a b}(\mathrm{Z}) d z^{a} d z^{b}\right]
\end{aligned}
$$

where $q_{\mu \nu}$ is the $(p+1)$-dimensional metric which depends only on the $(p+1)$-dimensional coordinates $x^{\mu}, \gamma_{i j}$ is the four-dimensional metric which depends only on the four-dimensional coordinates $y^{i}$, and finally $u_{a b}$ is the $(5-p)$-dimensional metric which depends only on the $(5-p)$-dimensional coordinates $z^{a}$.

We also assume that the scalar field $\phi$ and the gauge field strengths $F_{(p+2)}, F_{(p+6)}$ are given by

$$
\begin{aligned}
\mathrm{e}^{\phi} & =h_{p}^{(3-p) / 4} h_{p+4}^{-(1+p) / 4}, \\
F_{(p+2)} & =d\left[h_{p}^{-1}(x, y, z)\right] \wedge \Omega(\mathrm{X}), \\
F_{(p+6)} & =d\left[h_{p+4}^{-1}(z)\right] \wedge \Omega(\mathrm{X}) \wedge \Omega(\mathrm{Y}),
\end{aligned}
$$

where $\Omega(\mathrm{X})$ and $\Omega(\mathrm{Y})$ denote the volume $(p+1)$-, 4form, respectively

$$
\begin{aligned}
& \Omega(\mathrm{X})=\sqrt{-q} d x^{0} \wedge d x^{1} \wedge \cdots \wedge d x^{p} \\
& \Omega(\mathrm{Y})=\sqrt{\gamma} d y^{1} \wedge d y^{2} \wedge d y^{3} \wedge d y^{4}
\end{aligned}
$$

Here, $q$ and $\gamma$ are the determinant of the metrics $q_{\mu \nu}$ and $\gamma_{i j}$.

In terms of ansatz for fields (140) and (141), the field equations lead to

$$
\begin{gathered}
R_{\mu \nu}(\mathrm{X})=0, \quad R_{i j}(\mathrm{Y})=0, \quad R_{a b}(\mathrm{Z})=0, \\
h_{p}=h_{0}(x)+h_{1}(y, z), \quad D_{\mu} D_{\nu} h_{0}=0, \\
h_{p+4} \triangle_{\mathrm{Y}} h_{1}+\triangle_{\mathrm{Z}} h_{1}=0, \quad \triangle_{\mathrm{Z}} h_{p+4}=0,
\end{gathered}
$$

where $D_{\mu}$ is the covariant derivative with respect to the metric $q_{\mu \nu}$, and $\triangle_{\mathrm{Y}}, \triangle_{\mathrm{Z}}$ are the Laplace operators on $\mathrm{Y}, \mathrm{Z}$ space, and $R_{\mu \nu}(\mathrm{X}), R_{i j}(\mathrm{Y}), R_{a b}(\mathrm{Z})$ are the Ricci tensors associated with the metrics $q_{\mu \nu}(\mathrm{X}), \gamma_{i j}(\mathrm{Y}), u_{a b}(\mathrm{Z})$, respectively.
PHYSICAL REVIEW D 84, 126006 (2011)

Let us consider the case

$$
q_{\mu \nu}=\eta_{\mu \nu}, \quad \gamma_{i j}=\delta_{i j}, \quad u_{a b}=\delta_{a b},
$$

where $\eta_{\mu \nu}$ is the $(p+1)$-dimensional Minkowski metric and $\delta_{i j}, \delta_{a b}$ are the four-, $(5-p)$-dimensional Euclidean metrics, respectively. In the case of $p \neq 1, p \neq 3$, the solution for $h_{p}$ and $h_{p+4}$ can be expressed as

$$
\begin{aligned}
& \begin{array}{l}
h_{p}(x, y, z)= \\
\quad c_{\mu} x^{\mu}+\tilde{c} \\
\quad+\sum_{\ell} \frac{M_{\ell}}{\left[\left|\boldsymbol{y}-\boldsymbol{y}_{\ell}\right|^{2}+\frac{4 M}{(p-1)^{2}}\left|z-z_{0}\right|^{p-1}\right]^{p+1 / p-1}}, \\
h_{p+4}(z)=\frac{M}{\left|z-z_{0}\right|^{3-p}}, \\
\text { where } c_{\mu}, \tilde{c}, M_{\ell} \text { and } M \text { are constant parameters, and } \boldsymbol{y}_{\ell} \text { and }
\end{array} \\
& z_{0} \text { are constant vectors representing the positions of the } \\
& \text { branes. } \\
& \quad \text { For } p=1 \text {, we find } \\
& h_{1}(x, y, z)=c_{\mu} x^{\mu}+\tilde{c} \\
& \quad+\sum_{\ell} M_{\ell} \ln \left[\left|\boldsymbol{y}-\boldsymbol{y}_{\ell}\right|^{2}-4 M\left|z-z_{0}\right|\right], \text { (146a) } \\
& \qquad h_{5}(z)=\frac{M}{\left|z-z_{0}\right|^{2}} .
\end{aligned}
$$

If we consider the D3-D7 brane system, the solution is given by

$$
\begin{aligned}
h_{3}(x, y, z) & =c_{\mu} x^{\mu}+\tilde{c}+\sum_{\ell} \frac{M_{\ell}}{\left[\left|\boldsymbol{y}-\boldsymbol{y}_{\ell}\right|^{2}+M\left|z-z_{0}\right|^{2}\right]^{2}}, \\
h_{7}(z) & =M \ln \left|z-z_{0}\right| .
\end{aligned}
$$

\section{The intersection of Dp-brane and KK-monopole}

Now we discuss the KK-monopole in the transverse space of $\mathrm{D} p$-brane with $p \leq 4$. We assume that the tendimensional metric is given by

$$
\begin{aligned}
d s^{2}= & h^{(p-7) / 8}(x, y, z) q_{\mu \nu}(\mathrm{X}) d x^{\mu} d x^{\nu} \\
& +h^{(p+1) / 8}(x, y, z)\left[\gamma_{i j}(\mathrm{Y}) d y^{i} d y^{j}\right. \\
& \left.+h_{k}(z) u_{a b}(\mathrm{Z}) d z^{a} d z^{b}+h_{k}^{-1}(z)\left(d v+A_{a} d z^{a}\right)^{2}\right],
\end{aligned}
$$

where $q_{\mu \nu}$ is the $(p+1)$-dimensional metric which depends only on the $(p+1)$-dimensional coordinates $x^{\mu}, \gamma_{i j}$ is the $(5-p)$-dimensional metric which depends only on the $(5-p)$-dimensional coordinates $y^{i}$, and finally $u_{a b}$ is the three-dimensional metric which depends only on the three-dimensional coordinates $z^{a}$.

We also assume that the scalar field $\phi$ and the gauge field strength $F_{(p+2)}$ are given by 
DYNAMICS OF PARTIALLY LOCALIZED BRANE SYSTEMS

$$
\begin{aligned}
\mathrm{e}^{\phi} & =h^{(3-p) / 4}, \\
F_{(p+2)} & =d\left[h^{-1}(x, y, z)\right] \wedge \Omega(\mathrm{X}),
\end{aligned}
$$

where $\Omega(\mathrm{X})$ denotes the volume $(p+1)$-form

$$
\Omega(\mathrm{X})=\sqrt{-q} d x^{0} \wedge d x^{1} \wedge \cdots \wedge d x^{p} .
$$

Here, $q$ is the determinant of the metric $q_{\mu \nu}$.

Using the ansatz for fields (148) and (149), the field equations lead to

$$
\begin{array}{lrr}
R_{\mu \nu}(\mathrm{X})=0, & R_{i j}(\mathrm{Y})=0, \quad R_{a b}(\mathrm{Z})=0, & (151 \mathrm{a}) \\
h=h_{0}(x)+h_{1}(y, z), \quad d h_{k}=*_{\mathrm{Z}} d A, & (151 \mathrm{~b}) \\
D_{\mu} D_{\nu} h_{0}=0, & h_{k} \triangle_{\mathrm{Y}} h_{1}+\triangle_{\mathrm{Z}} h_{1}=0, & \triangle_{\mathrm{Z}} h_{k}=0,
\end{array}
$$

where $D_{\mu}$ is the covariant derivative with respect to the metric $q_{\mu \nu}$, and $\triangle_{\mathrm{Y}}, \triangle_{\mathrm{Z}}$ are the Laplace operators on $\mathrm{Y}, \mathrm{Z}$ space, and $R_{\mu \nu}(\mathrm{X}), R_{i j}(\mathrm{Y}), R_{a b}(\mathrm{Z})$ are the Ricci tensors associated with the metrics $q_{\mu \nu}(\mathrm{X}), \gamma_{i j}(\mathrm{Y}), u_{a b}(\mathrm{Z})$, respectively.

Now we set the metric:

$$
q_{\mu \nu}=\eta_{\mu \nu}, \quad \gamma_{i j}=\delta_{i j}, \quad u_{a b}=\delta_{a b},
$$

where $\eta_{\mu \nu}$ is the $(p+1)$-dimensional Minkowski metric and $\delta_{i j}, \delta_{a b}$ are the $(5-p)$-, three-dimensional Euclidean metrics, respectively. The solution for $h$ and $h_{k}$ can be obtained explicitly as

$$
\begin{aligned}
h(x, y, z) & =c_{\mu} x^{\mu}+\tilde{c}+\sum_{\ell} \frac{M_{\ell}}{\left[\left|\boldsymbol{y}-\boldsymbol{y}_{\ell}\right|^{2}+4 M\left|z-z_{0}\right|\right]^{(7-p) / 2}}, \\
h_{k}(z) & =\frac{M}{\left|z-z_{0}\right|},
\end{aligned}
$$

where $c_{\mu}, \tilde{c}, M_{\ell}$ and $M$ are constant parameters, and $\boldsymbol{y}_{\ell}$ and $z_{0}$ are constant vectors representing the positions of the branes.
PHYSICAL REVIEW D 84, 126006 (2011)

\section{E. The intersection of $\mathrm{D} p$-brane and plane wave}

We present the $\mathrm{D} p$-brane with the plane wave propagating along its longitudinal direction. We assume that the ten-dimensional metric takes the form

$$
\begin{aligned}
d s^{2}= & h^{(p-7) / 8}(z)\left[-d t^{2}+d x^{2}+\left\{h_{w}(t, y, z)-1\right\}(d t-d x)^{2}\right. \\
& \left.+\gamma_{i j}(\mathrm{Y}) d y^{i} d y^{j}+h(z) u_{a b}(\mathrm{Z}) d z^{a} d z^{b}\right]
\end{aligned}
$$

where $\gamma_{i j}$ is the $(p-1)$-dimensional metric which depends only on the $(p-1)$-dimensional coordinates $y^{i}$, and finally $u_{a b}$ is the $(9-p)$-dimensional metric which depends only on the $(9-p)$-dimensional coordinates $z^{a}$.

We also assume that the gauge field strength $F_{(p+2)}$ is given by

$$
\begin{aligned}
\mathrm{e}^{\phi} & =h^{(3-p) / 4}, \\
F_{(p+2)} & =d\left[h^{-1}(z) \wedge d t \wedge d x \wedge \Omega(\mathrm{Y})\right],
\end{aligned}
$$

where $\Omega(\mathrm{Y})$ denotes the volume ( $p-1)$-form

$$
\Omega(\mathrm{Y})=\sqrt{\gamma} d y^{1} \wedge d y^{2} \cdots \wedge d y^{p-1} .
$$

Here, $\gamma$ is the determinant of the metric $\gamma_{i j}$.

In terms of ansatz for fields (154) and (155), the field equations lead to

$$
\begin{aligned}
& R_{i j}(\mathrm{Y})=0, \quad R_{a b}(\mathrm{Z})=0, \\
& h_{w}=h_{0}(t)+h_{1}(y, z), \quad \partial_{t}^{2} h_{0}=0, \\
& h \triangle_{\mathrm{Y}} h_{1}+\triangle_{\mathrm{Z}} h_{1}=0, \quad \triangle_{\mathrm{Z}} h=0,
\end{aligned}
$$

where $\triangle_{\mathrm{Y}}, \triangle_{\mathrm{Z}}$ are the Laplace operators on $\mathrm{Y}, \mathrm{Z}$ space, and $R_{i j}(\mathrm{Y}), R_{a b}(\mathrm{Z})$ are the Ricci tensors associated with the metrics $\gamma_{i j}(\mathrm{Y}), u_{a b}(\mathrm{Z})$, respectively. Now we set the tendimensional metric

$$
\gamma_{i j}=\delta_{i j}, \quad u_{a b}=\delta_{a b},
$$

where $\delta_{i j}, \delta_{a b}$ are the four-dimensional Euclidean metrics, respectively. Using Eq. (158), the solution for $p \neq 5$ and $p \neq 7$ can be written as

$$
\begin{aligned}
h_{w}(t, y, z) & =\bar{c} t+\tilde{c}+\sum_{\ell} \frac{M_{\ell}}{\left[\left|\boldsymbol{y}-\boldsymbol{y}_{\ell}\right|^{2}+\frac{4 M}{(p-5)^{2}}\left|z-z_{0}\right|^{p-5}\right]^{p^{2}-8 p+19 / 2(p-5)}}, \\
h(z) & =\frac{M}{\left|z-z_{0}\right|^{7-p}},
\end{aligned}
$$

where $\bar{c}, \tilde{c}, M_{\ell}$ and $M$ are constant parameters, and $\boldsymbol{y}_{\ell}$ and $z_{0}$ are constant vectors representing the positions of the branes. The solution (159a) for $p=5$ becomes

$$
\begin{aligned}
h_{w}(t, y, z)= & \bar{c} t+\tilde{c} \\
& +\sum_{\ell} M_{\ell} \ln \left[\left|\boldsymbol{y}-\boldsymbol{y}_{\ell}\right|^{2}-(p-1) M\left|z-z_{0}\right|\right] \\
h(z)= & \frac{M}{\left|z-z_{0}\right|^{2}} .
\end{aligned}
$$

If we consider the case of $p=7$, the harmonic functions take the following form:

$$
\begin{aligned}
h_{w}(t, y, z) & =\bar{c} t+\tilde{c}+\sum_{\ell} \frac{M_{\ell}}{\left[\left|\boldsymbol{y}-\boldsymbol{y}_{\ell}\right|^{2}+M\left|z-z_{0}\right|^{2}\right]^{3}}, \\
h(z) & =M \ln \left|z-z_{0}\right| .
\end{aligned}
$$




\section{F. The pair intersection involving fundamental string}

Next we present intersecting fundamental string configurations of all the possible combinations. The basic constituents of intersecting branes are D-branes, fundamental string, solitonic NS5-brane, the KK-monopole and the plane wave.

\section{The intersection of $\mathrm{D} p$-brane and fundamental string}

First we discuss the $\mathrm{D} p$-branes ending on fundamental string. Let us consider the solution to be delocalized along the relative transverse direction of the $\mathrm{D} p$-branes. The tendimensional metric thus takes the form

$$
\begin{aligned}
d s^{2}= & h_{\mathrm{F}}^{-3 / 4}(t, y, z) h^{(p-7) / 8}(z) \\
& \times\left[-d t^{2}+h_{\mathrm{F}}(t, y, z) \gamma_{i j}(\mathrm{Y}) d y^{i} d y^{j}\right. \\
& \left.+h(z) d v^{2}+h_{\mathrm{F}}(t, y, z) h(z) u_{a b}(\mathrm{Z}) d z^{a} d z^{b}\right],
\end{aligned}
$$

where $\gamma_{i j}$ is the $p$-dimensional metric which depends only on the $p$-dimensional coordinates $y^{i}$, and finally $u_{a b}$ is the $(8-p)$-dimensional metric which depends only on the $(8-p)$-dimensional coordinates $z^{a}$.

The scalar field $\phi$ and the gauge field strength $H_{(3)}$ are also assumed to be

$$
\begin{aligned}
\mathrm{e}^{\phi} & =h_{\mathrm{F}}^{-1 / 2} h^{(3-p) / 4}, \\
H_{(3)} & =d\left[h_{\mathrm{F}}^{-1}(t, y, z)\right] \wedge d t \wedge d v, \\
F_{(p+2)} & =d\left[h^{-1}(z)\right] \wedge d t \wedge \Omega(\mathrm{Y}),
\end{aligned}
$$

where $\Omega(\mathrm{Y})$ denotes the volume $p$-form

$$
\Omega(\mathrm{Y})=\sqrt{\gamma} d y^{1} \wedge d y^{2} \wedge \cdots \wedge d y^{p}
$$

Here, $\gamma$ is the determinant of the metric $\gamma_{i j}$.

If we use ansatz for fields (162) and (163), the field equations give

$$
\begin{aligned}
& R_{i j}(\mathrm{Y})=0, \quad R_{a b}(\mathrm{Z})=0, \\
& h_{\mathrm{F}}=h_{0}(x)+h_{1}(y, z), \quad \partial_{t}^{2} h_{0}=0, \\
& h \triangle_{\mathrm{Y}} h_{1}+\triangle_{\mathrm{Z}} h_{1}=0, \quad \triangle_{\mathrm{Z}} h=0,
\end{aligned}
$$

where $\triangle_{\mathrm{Y}}, \triangle_{\mathrm{Z}}$ are the Laplace operators on $\mathrm{Y}, \mathrm{Z}$ space, and $R_{i j}(\mathrm{Y}), R_{a b}(\mathrm{Z})$ are the Ricci tensors associated with the metrics $\gamma_{i j}(\mathrm{Y}), u_{a b}(\mathrm{Z})$, respectively. We consider the case

$$
\gamma_{i j}=\delta_{i j}, \quad u_{a b}=\delta_{a b},
$$

where $\delta_{i j}, \delta_{a b}$ are the $p$-, $(8-p)$-dimensional Euclidean metrics, respectively. Under the metric Eq. (166), the solution of $h$ and $h_{\mathrm{F}}$ for $p=4$ and $p=6$ can be written by

$$
\begin{aligned}
h_{\mathrm{F}}(t, y, z)= & \bar{c} t+\tilde{c} \\
& +\sum_{\ell} \frac{M_{\ell}}{\left[\left|\boldsymbol{y}-\boldsymbol{y}_{\ell}\right|^{2}+\frac{4 M}{p-4}\left|z-z_{0}\right|^{p-4}\right]^{p^{2}-6 p+12 / 2(p-4)}}, \\
h(z)= & \frac{M}{\left|z-z_{0}\right|^{6-p}},
\end{aligned}
$$

where $\bar{c}, \tilde{c}, M_{\ell}$ and $M$ are constant parameters, and $\boldsymbol{y}_{\ell}$ and $z_{0}$ are constant vectors representing the positions of the branes. For $p=4$, the solution becomes

$$
\begin{aligned}
h_{\mathrm{F}}(t, y, z)= & \bar{c} t+\tilde{c} \\
& +\sum_{\ell} M_{\ell} \ln \left[\left|\boldsymbol{y}-\boldsymbol{y}_{\ell}\right|^{2}-p M\left|z-z_{0}\right|\right], \\
h(z)= & \frac{M}{\left|z-z_{0}\right|^{2}} .
\end{aligned}
$$

Next we consider the case of $p=6$. The solution is given by

$$
\begin{aligned}
h_{\mathrm{F}}(t, y, z) & =\bar{c} t+\tilde{c}+\sum_{\ell} \frac{M_{\ell}}{\left[\left|\boldsymbol{y}-\boldsymbol{y}_{\ell}\right|^{2}+M\left|z-z_{0}\right|^{2}\right]^{3}}, \\
h(z) & =M \ln \left|z-z_{0}\right| .
\end{aligned}
$$

\section{The intersection involving fundamental string and NS5-branes}

We discuss the solution of the fundamental strings with NS5-branes. We consider the case to be delocalized along one of the overall transverse directions. The tendimensional metric thus takes the form

$$
\begin{aligned}
d s^{2}= & h_{\mathrm{F}}^{-3 / 4}(x, y, z) h_{\mathrm{NS}}^{-1 / 4}(z) \\
& \times\left[q_{\mu \nu}(\mathrm{X})+h_{\mathrm{F}}(x, y, z) \gamma_{i j}(\mathrm{Y}) d y^{i} d y^{j}\right. \\
& \left.+h_{\mathrm{F}}(x, y, z) h_{\mathrm{NS}}(z) u_{a b}(\mathrm{Z}) d z^{a} d z^{b}\right],
\end{aligned}
$$

where $q_{\mu \nu}$ is the two-dimensional metric which depends only on the two-dimensional coordinates $x^{\mu}, \gamma_{i j}$ is the four-dimensional metric which depends only on the fourdimensional coordinates $y^{i}$, and finally $u_{a b}$ is the fourdimensional metric which depends only on the four-dimensional coordinates $z^{a}$.

We also assume that the scalar field $\phi$ and the gauge field strength $H_{(3)}$ are given by

$$
\begin{aligned}
\mathrm{e}^{\phi}= & h_{\mathrm{F}}^{-1 / 2} h_{\mathrm{NS}}^{1 / 2}, \\
H_{(3)}= & d\left[h_{\mathrm{F}}^{-1}(x, y, z)\right] \wedge \Omega(\mathrm{X}) \\
& +\mathrm{e}^{-\phi} * d\left[h_{\mathrm{NS}}^{-1}(z) \Omega(\mathrm{X}) \wedge \Omega(\mathrm{Y})\right],
\end{aligned}
$$

where $\Omega(\mathrm{X})$ and $\Omega(\mathrm{Y})$ denote the volume 2- and 4-form 


$$
\begin{aligned}
& \Omega(\mathrm{X})=\sqrt{-q} d x^{0} \wedge d x^{1} \\
& \Omega(\mathrm{Y})=\sqrt{\gamma} d y^{1} \wedge d y^{2} \wedge d y^{3} \wedge d y^{4} .
\end{aligned}
$$

Here, $q$ and $\gamma$ are the determinant of the metric $q_{\mu \nu}$ and $\gamma_{i j}$, respectively.

In terms of ansatz for fields (170) and (171), the field equations lead to

$$
\begin{gathered}
R_{\mu \nu}(\mathrm{X})=0, \quad R_{i j}(\mathrm{Y})=0, \quad R_{a b}(\mathrm{Z})=0, \\
h_{\mathrm{F}}=h_{0}(x)+h_{1}(y, z), \quad D_{\mu} D_{\nu} h_{0}=0, \\
h_{\mathrm{NS}} \triangle_{\mathrm{Y}} h_{1}+\triangle_{\mathrm{Z}} h_{1}=0, \quad \triangle_{\mathrm{Z}} h_{\mathrm{NS}}=0,
\end{gathered}
$$

where $D_{\mu}$ is the covariant derivative with respect to the metric $q_{\mu \nu}$, and $\triangle_{\mathrm{Y}}, \triangle_{\mathrm{Z}}$ are the Laplace operators on $\mathrm{Y}, \mathrm{Z}$ space, and $R_{i j}(\mathrm{X}), R_{i j}(\mathrm{Y}), R_{a b}(\mathrm{Z})$ are the Ricci tensors associated with the metrics $q_{\mu \nu}(\mathrm{X}), \gamma_{i j}(\mathrm{Y}), u_{a b}(\mathrm{Z})$, respectively. We assume that the ten-dimensional metric is given by

$$
q_{\mu \nu}=\eta_{\mu \nu}, \quad \gamma_{i j}=\delta_{i j}, \quad u_{a b}=\delta_{a b},
$$

where $\eta_{\mu \nu}$ is the two-dimensional Minkowski metric and $\delta_{i j}, \delta_{a b}$ are the four-dimensional Euclidean metrics, respectively. The solution for $h_{\mathrm{F}}$ and $h_{\mathrm{NS}}$ can be obtained explicitly as

$$
\begin{aligned}
h_{\mathrm{F}}(x, y, z)= & c_{\mu} x^{\mu}+\tilde{c} \\
& +\sum_{\ell} M_{\ell} \ln \left[\left|\boldsymbol{y}-\boldsymbol{y}_{\ell}\right|^{2}-4 M\left|z-z_{0}\right|\right], \\
h_{\mathrm{NS}}(z)= & \frac{M}{\left|z-z_{0}\right|^{2}},
\end{aligned}
$$

where $c_{\mu}, \tilde{c}, M_{\ell}$ and $M$ are constant parameters, and $\boldsymbol{y}_{\ell}$ and $z_{0}$ are constant vectors representing the positions of the branes. If we delocalize the solution along one of the overall transverse directions, the solution can be written as

$$
\begin{aligned}
h_{\mathrm{F}}(x, y, z) & =c_{\mu} x^{\mu}+\tilde{c}+\sum_{\ell} \frac{M_{\ell}}{\left[\left|\boldsymbol{y}-\boldsymbol{y}_{\ell}\right|^{2}+4 M\left|z-z_{0}\right|\right]^{3}}, \\
h_{\mathrm{NS}}(z) & =\frac{M}{\left|z-z_{0}\right|} .
\end{aligned}
$$

\section{The pair involving fundamental string and one Kaluza- Klein monopole}

Now we discuss the KK-monopole in the transverse space of the fundamental string. We set the tendimensional metric takes the form

$$
\begin{aligned}
d s^{2}= & h_{\mathrm{F}}^{-3 / 4}(x, y, z) q_{\mu \nu}(\mathrm{X}) d x^{\mu} d x^{\nu} \\
& +h_{\mathrm{F}}^{1 / 4}(x, y, z)\left[\gamma_{i j}(\mathrm{Y}) d y^{i} d y^{j}\right. \\
& \left.+h_{k}(z) u_{a b}(\mathrm{Z}) d z^{a} d z^{b}+h_{k}^{-1}(z)\left(d v+A_{a} d z^{a}\right)^{2}\right],
\end{aligned}
$$

where $q_{\mu \nu}$ is the two-dimensional metric which depends only on the two-dimensional coordinates $x^{\mu}, \gamma_{i j}$ is the four-dimensional metric which depends only on the fourdimensional coordinates $y^{i}$, and finally $u_{a b}$ is the threedimensional metric which depends only on the threedimensional coordinates $z^{a}$.

We assume that the scalar field $\phi$ and the gauge field strength $H_{(3)}$ are given by

$$
\begin{aligned}
\mathrm{e}^{\phi} & =h_{\mathrm{F}}^{-1 / 2}, \\
H_{(3)} & =d\left[h_{\mathrm{F}}^{-1}(x, y, z)\right] \wedge \Omega(\mathrm{X}),
\end{aligned}
$$

where $\Omega(\mathrm{X})$ denotes the volume 2 -form

$$
\Omega(\mathrm{X})=\sqrt{-q} d x^{0} \wedge d x^{1} .
$$

Here, $q$ is the determinant of the metric $q_{\mu \nu}$.

In terms of ansatz for fields (177) and (178), the field equations lead to

$$
\begin{gathered}
R_{\mu \nu}(\mathrm{X})=0, \quad R_{i j}(\mathrm{Y})=0, \quad R_{a b}(\mathrm{Z})=0, \\
h_{\mathrm{F}}=h_{0}(x)+h_{1}(y, z), \quad D_{\mu} D_{\nu} h_{0}=0, \\
h_{k} \triangle_{\mathrm{Y}} h_{1}+\triangle_{\mathrm{Z}} h_{1}=0, \quad \triangle_{\mathrm{Z}} h_{k}=0,
\end{gathered}
$$

where $D_{\mu}$ is the covariant derivative with respect to the metric $q_{\mu \nu}$, and $\triangle_{\mathrm{Y}}, \triangle_{\mathrm{Z}}$ are the Laplace operators on $\mathrm{Y}, \mathrm{Z}$ space, and $R_{i j}(\mathrm{X}), R_{i j}(\mathrm{Y}), R_{a b}(\mathrm{Z})$ are the Ricci tensors associated with the metrics $q_{\mu \nu}(\mathrm{X}), \gamma_{i j}(\mathrm{Y}), u_{a b}(\mathrm{Z})$, respectively.

Now let us consider the case

$$
q_{\mu \nu}=\eta_{\mu \nu}, \quad \gamma_{i j}=\delta_{i j}, \quad u_{a b}=\delta_{a b},
$$

where $\eta_{\mu \nu}$ is the two-dimensional Minkowski metric and $\delta_{i j}, \delta_{a b}$ are the four-, three-dimensional Euclidean metrics, respectively. The solution for $h_{\mathrm{F}}$ and $h_{k}$ can be obtained explicitly as

$$
\begin{aligned}
h_{\mathrm{F}}(x, y, z) & =c_{\mu} x^{\mu}+\tilde{c}+\sum_{\ell} \frac{M_{\ell}}{\left[\left|\boldsymbol{y}-\boldsymbol{y}_{\ell}\right|^{2}+4 M\left|z-z_{0}\right|\right]^{3}}, \\
h_{k}(z) & =\frac{M}{\left|z-z_{0}\right|},
\end{aligned}
$$

where $c_{\mu}, \tilde{c}, M_{\ell}$ and $M$ are constant parameters, and $\boldsymbol{y}_{\ell}$ and $z_{0}$ are constant vectors representing the positions of the branes. 


\section{G. The intersection involving NS5-branes}

In this subsection, we discuss the intersecting brane involving NS5-branes. For the KK-monopole, we cannot find partially delocalized solutions because the intersection does not have the relative transverse directions.

\section{The intersection involving $\mathrm{D} p$-brane and NS5-branes}

Let us first consider the D p-branes ending on NS5branes. We discuss the solution to be delocalized along the relative transverse direction of the NS5-branes. The ten-dimensional metric of $\mathrm{D} p$-branes $(p \leq 6)$ ending on NS5-branes thus takes the form

$$
\begin{aligned}
d s^{2}= & h^{(p-7) / 8}(x, y, z) h_{\mathrm{NS}}^{-1 / 4}(z)\left[q_{\mu \nu}(\mathrm{X}) d x^{\mu} d x^{\nu}\right. \\
& +h(x, y, z) \gamma_{i j}(\mathrm{Y}) d y^{i} d y^{j}+h_{\mathrm{NS}}(z) d v^{2} \\
& \left.+h(x, y, z) h_{\mathrm{NS}}(z) u_{a b}(\mathrm{Z}) d z^{a} d z^{b}\right],
\end{aligned}
$$

where $q_{\mu \nu}$ is the $p$-dimensional metric which depends only on the $p$-dimensional coordinates $x^{\mu}, \gamma_{i j}$ is the $(6-p)$-dimensional metric which depends only on the $(6-p)$-dimensional coordinates $y^{i}$, and finally $u_{a b}$ is the three-dimensional metric which depends only on the three-dimensional coordinates $z^{a}$.

We assume that the scalar field $\phi$ and the gauge field strength $H_{(3)}$ are given by

$$
\begin{aligned}
\mathrm{e}^{\phi} & =h_{\mathrm{NS}}^{1 / 2} h^{(3-p) / 4}, \\
H_{(3)} & =\mathrm{e}^{-\phi} * d\left[h_{\mathrm{NS}}^{-1}(z) \Omega(\mathrm{X}) \wedge \Omega(\mathrm{Y})\right], \\
F_{(p+2)} & =d\left[h^{-1}(x, y, z)\right] \wedge \Omega(\mathrm{X}),
\end{aligned}
$$

where $\Omega(\mathrm{X})$ and $\Omega(\mathrm{Y})$ denote the volume $p$-form, $(6-p)$ form, respectively

$$
\begin{aligned}
& \Omega(\mathrm{X})=\sqrt{-q} d x^{0} \wedge d x^{1} \wedge \cdots \wedge d x^{p-1}, \\
& \Omega(\mathrm{Y})=\sqrt{\gamma} d y^{1} \wedge d y^{2} \wedge \cdots \wedge d y^{6-p} .
\end{aligned}
$$

Here, $q$ and $\gamma$ are the determinant of the metric $q_{\mu \nu}, \gamma_{i j}$, respectively.

Using the ansatz for fields (183) and (184), the field equations lead to

$$
\begin{gathered}
R_{\mu \nu}(\mathrm{X})=0, \quad R_{i j}(\mathrm{Y})=0, \quad R_{a b}(\mathrm{Z})=0, \\
h=h_{0}(x)+h_{1}(z), \quad D_{\mu} D_{\nu} h_{0}=0, \\
h_{\mathrm{NS}} \triangle_{\mathrm{Y}} h_{1}+\triangle_{\mathrm{Z}} h_{1}=0, \quad \triangle_{\mathrm{Z}} h_{\mathrm{NS}}=0,
\end{gathered}
$$

where $D_{\mu}$ is the covariant derivative constructed by the metric $q_{\mu \nu}$, and $\triangle_{\mathrm{Y}}, \triangle_{\mathrm{Z}}$ are the Laplace operators on $\mathrm{Y}, \mathrm{Z}$ space, and $R_{\mu \nu}(\mathrm{X}), R_{i j}(\mathrm{Y}), R_{a b}(\mathrm{Z})$ are the Ricci tensors associated with the metrics $q_{\mu \nu}(\mathrm{X}), \gamma_{i j}(\mathrm{Y}), u_{a b}(\mathrm{Z})$, respectively. Let us consider the case

$$
q_{\mu \nu}=\eta_{\mu \nu}, \quad \gamma_{i j}=\delta_{i j}, \quad u_{a b}=\delta_{a b},
$$

where $\eta_{\mu \nu}$ is the $p$-dimensional Minkowski metric and $\delta_{i j}$, $\delta_{a b}$ are the $(6-p)$-, three-dimensional Euclidean metrics, respectively. The solution for $h$ and $h_{\mathrm{NS}}$ can be obtained explicitly as

$$
\begin{aligned}
h(x, y, z)= & c_{\mu} x^{\mu}+\tilde{c} \\
& +\sum_{\ell} \frac{M_{\ell}}{\left[\left|\boldsymbol{y}-\boldsymbol{y}_{\ell}\right|^{2}+4 M\left|z-z_{0}\right|\right]^{(p-8) / 2}}, \\
h_{\mathrm{NS}}(z)= & \frac{M}{\left|z-z_{0}\right|},
\end{aligned}
$$

where $c_{\mu}, \tilde{c}, M_{\ell}$ and $M$ are constant parameters, and $\boldsymbol{y}_{\ell}$ and $z_{0}$ are constant vectors representing the positions of the branes.

\section{The intersection of two NS5-branes}

Next we consider the solution of two NS5-brane. As we mentioned in Sec. II A, these intersect over three dimensions. We assume that the ten-dimensional metric is written by

$$
\begin{aligned}
d s^{2}= & h_{\mathrm{NS}}^{-1 / 4}(x, y, z) \bar{h}_{\mathrm{NS}}^{-1 / 4}(z)\left[q_{\mu \nu}(\mathrm{X}) d x^{\mu} d x^{\nu}\right. \\
& +h_{\mathrm{NS}}(x, y, z) \gamma_{i j}\left(\mathrm{Y}_{1}\right) d y^{i} d y^{j} \\
& +\bar{h}_{\mathrm{NS}}(z) w_{m n}\left(\mathrm{Y}_{2}\right) d v^{m} d v^{n} \\
& \left.+h_{\mathrm{NS}}(x, y, z) \bar{h}_{\mathrm{NS}}(z) u_{a b}(\mathrm{Z}) d z^{a} d z^{b}\right]
\end{aligned}
$$

where $q_{\mu \nu}$ is the four-dimensional metric which depends only on the four-dimensional coordinates $x^{\mu}, \gamma_{i j}$ is the two-dimensional metric which depends only on the twodimensional coordinates $y^{i}, w_{m n}$ is the two-dimensional metric which depends only on the two-dimensional coordinates $v^{m}$, and finally $u_{a b}$ is the two-dimensional metric which depends only on the two-dimensional coordinates $z^{a}$.

We also assume that the scalar field $\phi$ and the gauge field strength $H_{(3)}$ are given by

$$
\begin{aligned}
\mathrm{e}^{\phi}= & \left(h_{\mathrm{NS}} \bar{h}_{\mathrm{NS}}\right)^{1 / 2}, \\
H_{(3)}= & \mathrm{e}^{-\phi} * d\left[h_{\mathrm{NS}}^{-1}(x, y, z) \Omega(\mathrm{X}) \wedge \Omega\left(\mathrm{Y}_{2}\right)\right. \\
& \left.+\bar{h}_{\mathrm{NS}}^{-1}(z) \Omega(\mathrm{X}) \wedge \Omega\left(\mathrm{Y}_{1}\right)\right],
\end{aligned}
$$

where $\Omega(\mathrm{X}), \Omega\left(\mathrm{Y}_{1}\right)$ and $\Omega\left(\mathrm{Y}_{2}\right)$ denote the volume 4-form, 4-form and 4-form, respectively

$$
\begin{aligned}
\Omega(\mathrm{X}) & =\sqrt{-q} d x^{0} \wedge d x^{1} \wedge d x^{2} \wedge d x^{3} \\
\Omega\left(\mathrm{Y}_{1}\right) & =\sqrt{\gamma} d y^{1} \wedge d y^{2} \\
\Omega\left(\mathrm{Y}_{2}\right) & =\sqrt{w} d v^{1} \wedge d v^{2}
\end{aligned}
$$

Here, $q, \gamma$ and $w$ are the determinants of the metrics $q_{\mu \nu}$, $\gamma_{i j}$, and $w_{m n}$, respectively.

In terms of ansatz for fields (189) and (190), the field equations lead to 


$$
\begin{aligned}
& R_{\mu \nu}(\mathrm{X})=0, \quad R_{i j}\left(\mathrm{Y}_{1}\right)=0, \\
& \quad R_{m n}\left(\mathrm{Y}_{2}\right)=0, \quad R_{a b}(\mathrm{Z})=0, \\
& h_{\mathrm{NS}}=h_{0}(x)+h_{1}(y, z), \\
& D_{\mu} D_{\nu} h_{0}=0, \quad \bar{h}_{\mathrm{NS}} \triangle_{\mathrm{Y}} h_{1}+\triangle_{\mathrm{Z}} h_{1}=0,
\end{aligned}
$$

where $D_{\mu}$ is the covariant derivative constructed by the metric $q_{\mu \nu}$, and $\triangle_{\mathrm{Y}}, \triangle_{\mathrm{Z}}$ are the Laplace operators on $\mathrm{Y}, \mathrm{Z}$ space, and $R_{\mu \nu}(\mathrm{X}), R_{m n}(\mathrm{Y}), R_{a b}(\mathrm{Z})$ are the Ricci tensors associated with the metrics $q_{\mu \nu}(\mathrm{X}), \gamma_{i j}(\mathrm{Y}), u_{a b}(\mathrm{Z})$, respectively. As a special example, we consider the case

$$
q_{\mu \nu}=\eta_{\mu \nu}, \quad \gamma_{i j}=\delta_{i j}, \quad u_{a b}=\delta_{a b},
$$

where $\eta_{\mu \nu}$ is the four-dimensional Minkowski metric and $\delta_{i j}, \delta_{m n}, \delta_{a b}$ are the two-, two-, two-dimensional Euclidean metrics, respectively. The solution for $h_{\mathrm{NS}}$ and $\bar{h}_{\mathrm{NS}}$ can be obtained explicitly as

$h_{\mathrm{NS}}(x, y, z)=c_{\mu} x^{\mu}+\tilde{c}+\sum_{\ell} \frac{M_{\ell}}{\left|\boldsymbol{y}-\boldsymbol{y}_{\ell}\right|^{2}+M\left|z-z_{0}\right|^{2}}$,

$$
\bar{h}_{\mathrm{NS}}(z)=\ln \left[M\left|z-z_{0}\right|\right]
$$

where $c_{\mu}, \tilde{c}, M_{\ell}$ and $M$ are constant parameters, and $\boldsymbol{y}_{\ell}$ and $z_{0}$ are constant vectors representing the positions of the branes.

\section{The intersection involving plane wave and NS5-brane}

We present the NS5-brane with the plane wave propagating along its longitudinal direction. We assume that the ten-dimensional metric takes the form

$$
\begin{aligned}
d s^{2}= & h_{\mathrm{NS}}^{-1 / 4}(z)\left[-d t^{2}+d x^{2}+\left\{h_{w}(t, y, z)-1\right\}(d t-d x)^{2}\right. \\
& \left.+\gamma_{i j}(\mathrm{Y}) d y^{i} d y^{j}+h_{\mathrm{NS}}(z) u_{a b}(\mathrm{Z}) d z^{a} d z^{b}\right],
\end{aligned}
$$

where $\gamma_{i j}$ is the four-dimensional metric which depends only on the four-dimensional coordinates $y^{i}$, and finally $u_{a b}$ is the four-dimensional metric which depends only on the four-dimensional coordinates $z^{a}$.

We set the scalar field $\phi$ and the gauge field strength $H_{(3)}$ as follows:

$$
\begin{aligned}
\mathrm{e}^{\phi} & =h_{\mathrm{NS}}^{1 / 2}, \\
H_{(3)} & =\mathrm{e}^{-\phi} * d\left[h_{\mathrm{NS}}^{-1}(z) \wedge d t \wedge d x \wedge \Omega(\mathrm{Y})\right],
\end{aligned}
$$

where $\Omega(\mathrm{Y})$ denotes the volume 4-form

$$
\Omega(\mathrm{Y})=\sqrt{\gamma} d y^{1} \wedge d y^{2} \wedge d y^{3} \wedge d y^{4} .
$$

Here, $\gamma$ is the determinant of the metric $\gamma_{i j}$.

In terms of ansatz for fields (195) and (196), the field equations lead to

$$
\begin{aligned}
& R_{i j}(\mathrm{Y})=0, \quad R_{a b}(\mathrm{Z})=0, \\
& h_{w}=h_{0}(t)+h_{1}(y, z), \quad \partial_{t}^{2} h_{0}=0, \\
& \quad h_{k} \triangle_{\mathrm{Y}} h_{1}+\triangle_{\mathrm{Z}} h_{1}=0, \quad \triangle_{\mathrm{Z}} h_{\mathrm{NS}}=0,
\end{aligned}
$$

where $\triangle_{\mathrm{Y}}, \triangle_{\mathrm{Z}}$ are the Laplace operators on $\mathrm{Y}, \mathrm{Z}$ space, and $R_{i j}(\mathrm{Y}), R_{a b}(\mathrm{Z})$ are the Ricci tensors associated with the metrics $\gamma_{i j}(\mathrm{Y}), u_{a b}(\mathrm{Z})$, respectively. As a special example, we set the metric and the function $h_{\mathrm{NS}}$

$$
\gamma_{i j}=\delta_{i j}, \quad u_{a b}=\delta_{a b},
$$

where $\delta_{i j}, \delta_{a b}$ are the four-dimensional Euclidean metrics, respectively. The solution for $h_{\mathrm{NS}}$ and $h_{w}$ can be obtained explicitly as

$$
\begin{aligned}
h_{w}(t, y, z) & =\bar{c} t+\tilde{c}+\sum_{\ell} M_{\ell} \ln \left[\left|\boldsymbol{y}-\boldsymbol{y}_{\ell}\right|^{2}-4 M\left|z-z_{0}\right|\right], \\
h_{\mathrm{NS}}(z) & =\frac{M}{\left|z-z_{0}\right|^{2}},
\end{aligned}
$$

where $\bar{c}, \tilde{c}, M_{\ell}$ and $M$ are constant parameters, and $\boldsymbol{y}_{\ell}$ and $z_{0}$ are constant vectors representing the positions of the branes.

\section{H. The plane wave in the KK-monopole background}

We consider the plane wave propagating in the background of the KK-monopole. The solution of tendimensional metric is given by

$$
\begin{aligned}
d s^{2}= & -d t^{2}+d x^{2}+\left[h_{w}(t, y, z)-1\right](d t-d x)^{2} \\
& +\gamma_{i j}(\mathrm{Y}) d y^{i} d y^{j}+h_{k}(z) u_{a b}(\mathrm{Z}) d z^{a} d z^{b} \\
& +h_{k}^{-1}(z)\left(d v+A_{a} d z^{a}\right)^{2},
\end{aligned}
$$

where $\gamma_{i j}$ is the four-dimensional metric which depends only on the four-dimensional coordinates $y^{i}$, and finally $u_{a b}$ is the three-dimensional metric which depends only on the three-dimensional coordinates $z^{a}$.

The ten-dimensional metric and the function $h_{k}$ obey

$$
\begin{aligned}
& R_{i j}(\mathrm{Y})=0, \quad R_{a b}(\mathrm{Z})=0, \\
& h_{w}=h_{0}(t)+h_{1}(y, z), \quad \partial_{t}^{2} h_{0}=0, \\
& \quad h_{k} \triangle_{\mathrm{Y}} h_{1}+\triangle_{\mathrm{Z}} h_{1}=0, \quad \triangle_{\mathrm{Z}} h_{k}=0, \\
& d h_{k}=*_{\mathrm{Z}} d A,
\end{aligned}
$$

where $*_{\mathrm{Z}}$ is the Hodge operator in the $\mathrm{Z}$ space, and $\triangle_{\mathrm{Y}}, \triangle_{\mathrm{Z}}$ are the Laplace operators on $\mathrm{Y}, \mathrm{Z}$ space, and $R_{i j}(\mathrm{Y}), R_{a b}(\mathrm{Z})$ are the Ricci tensors associated with the metrics $\gamma_{i j}(\mathrm{Y})$, $u_{a b}(\mathrm{Z})$, respectively. Now we consider the case

$$
\gamma_{i j}=\delta_{i j}, \quad u_{a b}=\delta_{a b},
$$

where $\delta_{i j}, \delta_{a b}$ are the four-, three-dimensional Euclidean metrics, respectively. The solution for $h$ and $h_{k}$ can be obtained explicitly as 


$$
\begin{aligned}
h(t, y, z) & =\bar{c} t+\tilde{c}+\sum_{\ell} \frac{M_{\ell}}{\left[\left|\boldsymbol{y}-\boldsymbol{y}_{\ell}\right|^{2}+4 M\left|z-z_{0}\right|\right]^{3}}, \\
h_{k}(z) & =\frac{M}{\left|z-z_{0}\right|},
\end{aligned}
$$

where $\bar{c}, \tilde{c}, M_{\ell}$ and $M$ are constant parameters, and $\boldsymbol{y}_{\ell}$ and $z_{0}$ are constant vectors representing the positions of the branes.

There is a classification of the multiple intersecting branes solutions by [27,28]. The dynamical delocalized branes in ten-dimensional theory are also classified in [4]. We again show the intersection rule for the branes with M-wave and KK-monopoles in Table IV. In the Table, circles indicate where the brane world-volumes enter, $v$ represents the coordinate of the KK-monopole, and the time-dependent branes are indicated by $\sqrt{ }$ for different solutions.

\section{COSMOLOGY}

In this section, we apply the above solutions to study the four-dimensional cosmology. We assume an isotropic and homogeneous three-space in the four-dimensional spacetime known as Friedmann-Lemaître-Robertson-Walker (FLRW) universe after compactification. In what follows, we concentrate on the $(p+1)$-dimensional Minkowski spacetime with $q_{\mu \nu}(\mathrm{X})=\eta_{\mu \nu}(\mathrm{X})$, and drop the coordinate dependence on $\mathrm{X}$ space except for the time. We discuss just the cases involving $p$-brane and KK-monopole because our
Universe does not expand when the wave is timedependent. Hence, we have no interesting case for wave solution.

\section{A. The intersection of $\mathrm{D} p_{r}-\mathrm{D} p_{s}$ brane system}

Let us first discuss how these solutions are applied to our physical world in the case of $\mathrm{D} p_{r}-\mathrm{D} p_{s}$ brane system. Suppose that our Universe is a part of branes. Since our Universe is isotropic and homogeneous, same branes must contain this whole three dimensions. The $D$-dimensional metric (5) can be expressed as

$$
d s^{2}=-h d t^{2}+d s^{2}(\tilde{\mathrm{X}})+d s^{2}\left(\mathrm{Y}_{1}\right)+d s^{2}\left(\mathrm{Y}_{2}\right)+d s^{2}(\mathrm{Z}),
$$

where we have defined

$$
\begin{aligned}
d s^{2}(\tilde{\mathrm{X}}) & \equiv h \delta_{P Q}(\tilde{\mathrm{X}}) d \theta^{P} d \theta^{Q} \\
d s^{2}\left(\mathrm{Y}_{1}\right) & \equiv h_{r}^{b_{r}}(t, y, z) h_{s}^{a_{s}}(t, v, z) \gamma_{i j}\left(\mathrm{Y}_{1}\right) d y^{i} d y^{j} \\
d s^{2}\left(\mathrm{Y}_{2}\right) & \equiv h_{r}^{a_{r}}(t, y, z) h_{s}^{b_{s}}(t, v, z) w_{m n}\left(\mathrm{Y}_{2}\right) d v^{m} d v^{n} \\
d s^{2}(\mathrm{Z}) & \equiv h_{r}^{b_{r}}(t, y, z) h_{s}^{b_{s}}(t, v, z) u_{a b}(\mathrm{Z}) d z^{a} d z^{b} \\
h & \equiv h_{r}^{a_{r}}(t, y, z) h_{s}^{a_{s}}(t, v, z)
\end{aligned}
$$

Here, $\delta_{P Q}(\tilde{\mathrm{X}})$ is the $p$-dimensional Euclidean metric, and $\theta^{P}$ denotes the coordinate of the $p$-dimensional Euclid space $\tilde{X}$.

In the following, we assume $h_{s}=h_{s}(z)$ and set $h_{r}=$ $A t+h_{1}(y, z)$. The $D$-dimensional metric (206) can be written as

$$
\begin{aligned}
d s^{2}= & h_{s}^{a_{s}}\left[1+\left(\frac{\tau}{\tau_{0}}\right)^{-2 /\left(a_{r}+2\right)} h_{1}\right]^{a_{r}}\left[-d \tau^{2}+\left(\frac{\tau}{\tau_{0}}\right)^{2 a_{r} /\left(a_{r}+2\right)} \delta_{P Q}(\tilde{\mathrm{X}}) d \theta^{P} d \theta^{Q}\right. \\
& +\left\{1+\left(\frac{\tau}{\tau_{0}}\right)^{-2 /\left(a_{r}+2\right)} h_{1}\right\}\left(\frac{\tau}{\tau_{0}}\right)^{2 b_{r} /\left(a_{r}+2\right)} \gamma_{i j}\left(\mathrm{Y}_{1}\right) d y^{i} d y^{j}+h_{s}\left(\frac{\tau}{\tau_{0}}\right)^{2 a_{r} /\left(a_{r}+2\right)} w_{m n}\left(\mathrm{Y}_{2}\right) d v^{m} d v^{n} \\
& \left.+h_{s}\left\{1+\left(\frac{\tau}{\tau_{0}}\right)^{-2 /\left(a_{r}+2\right)} h_{1}\right\}\left(\frac{\tau}{\tau_{0}}\right)^{2 b_{r} /\left(a_{r}+2\right)} u_{a b}(\mathrm{Z}) d z^{a} d z^{b}\right],
\end{aligned}
$$

where we have introduced the cosmic time $\tau$ defined by

$$
\frac{\tau}{\tau_{0}}=(A t)^{\left(a_{r}+2\right) / 2}, \quad \tau_{0}=\frac{2}{\left(a_{r}+2\right) A} .
$$

On the other hand, for $h_{r}=h_{r}(z)$ and $h_{s}=A t+k_{1}(v, z)$, the metric (206) is given by replacing $a_{r}$ and $h_{1}(y, z)$ with $a_{s}$ and $k_{1}(v, z)$.

Now we apply these solutions to lower-dimensional effective theory. We compactify $d\left(\equiv d_{1}+d_{2}+d_{3}+d_{4}\right)$ dimensions to fit our Universe, where $d_{1}, d_{2}, d_{3}$, and $d_{4}$ denote the compactified dimensions with respect to the $\tilde{X}$, $\mathrm{Y}_{1}, \mathrm{Y}_{2}$, and $\mathrm{Z}$ spaces. The metric (205) is then described by

$$
d s^{2}=d s^{2}(\mathrm{M})+d s^{2}(\mathrm{~N})
$$

where $d s^{2}(\mathrm{M})$ is the $(D-d)$-dimensional metric and $d s^{2}(\mathrm{~N})$ is the metric of compactified dimensions.

By the conformal transformation

$$
d s^{2}(\mathrm{M})=h_{r}^{B} h_{s}^{C} d s^{2}(\overline{\mathrm{M}}),
$$

we can rewrite the $(D-d)$-dimensional metric in the Einstein frame. Here $B$ and $C$ are

$$
\begin{aligned}
& B=\frac{-\left(a_{r}+1\right) d+d_{1}+d_{3}}{D-d-2}, \\
& C=\frac{-\left(a_{s}+1\right) d+d_{2}+d_{4}}{D-d-2} .
\end{aligned}
$$

Hence, the $(D-d)$-dimensional metric in the Einstein frame is 


$$
\begin{aligned}
d s^{2}(\overline{\mathrm{M}})= & h_{r}^{B^{\prime}} h_{s}^{C^{\prime}}\left[-d t^{2}+\delta_{P^{\prime} Q^{\prime}}\left(\tilde{\mathrm{X}}^{\prime}\right) d \theta^{P^{\prime}} d \theta^{Q^{\prime}}\right. \\
& +h_{r} \gamma_{k^{\prime} l^{\prime}}\left(\mathrm{Y}_{1}^{\prime}\right) d y^{k^{\prime}} d y^{l^{\prime}} \\
& +h_{s} w_{m^{\prime} n^{\prime}}\left(\mathrm{Y}_{2}^{\prime}\right) d v^{m^{\prime}} d v^{n^{\prime}} \\
& \left.+h_{r} h_{s} u_{a^{\prime} b^{\prime}}\left(\mathrm{Z}^{\prime}\right) d z^{a^{\prime}} d z^{b^{\prime}}\right],
\end{aligned}
$$

where $B^{\prime}$ and $C^{\prime}$ are defined by $B^{\prime}=-B+a_{r}$ and $C^{\prime}=$ $-C+a_{s}$, and $\tilde{\mathrm{X}}^{\prime}, \mathrm{Y}_{1}^{\prime}, \mathrm{Y}_{2}^{\prime}$, and $\mathrm{Z}^{\prime}$ denote the $\left(p-d_{1}\right)$-, $\left(p_{s}-p-d_{2}\right)-,\left(p_{r}-p-d_{3}\right)-$, and $\left(D+p-p_{r}-p_{s}-\right.$ $\left.d_{4}\right)$-dimensional spaces, respectively.

For $h_{r}=A t+h_{1}$, the metric (212) is thus rewritten as

$$
\begin{aligned}
d s^{2}(\overline{\mathrm{M}})= & h_{s}^{C^{\prime}}\left[1+\left(\frac{\tau}{\tau_{0}}\right)^{-2 /\left(B^{\prime}+2\right)} h_{1}\right]^{B^{\prime}}\left[-d \tau^{2}+\left(\frac{\tau}{\tau_{0}}\right)^{2 B^{\prime} /\left(B^{\prime}+2\right)} \delta_{P^{\prime} Q^{\prime}}\left(\tilde{\mathrm{X}}^{\prime}\right) d \theta^{P^{\prime}} d \theta^{Q^{\prime}}\right. \\
& +\left\{1+\left(\frac{\tau}{\tau_{0}}\right)^{-2 /\left(B^{\prime}+2\right)} h_{1}\right\}\left(\frac{\tau}{\tau_{0}}\right)^{2\left(B^{\prime}+1\right) /\left(B^{\prime}+2\right)} \gamma_{k^{\prime} l^{\prime}}\left(\mathrm{Y}_{1}^{\prime}\right) d y^{k^{\prime}} d y^{l^{\prime}}+h_{s}\left(\frac{\tau}{\tau_{0}}\right)^{2 B^{\prime} /\left(B^{\prime}+2\right)} w_{m^{\prime} n^{\prime}}\left(\mathrm{Y}_{2}^{\prime}\right) d v^{m^{\prime}} d v^{n^{\prime}} \\
& \left.+h_{s}\left\{1+\left(\frac{\tau}{\tau_{0}}\right)^{-2 /\left(B^{\prime}+2\right)} h_{1}\right\}\left(\frac{\tau}{\tau_{0}}\right)^{2\left(B^{\prime}+1\right) /\left(B^{\prime}+2\right)} u_{a^{\prime} b^{\prime}}\left(\mathrm{Z}^{\prime}\right) d z^{a^{\prime}} d z^{b^{\prime}}\right]
\end{aligned}
$$

where the cosmic time $\tau$ is defined by

$$
\frac{\tau}{\tau_{0}}=(A t)^{\left(B^{\prime}+2\right) / 2}, \quad \tau_{0}=\frac{2}{\left(B^{\prime}+2\right) A} .
$$

For $h_{s}=A t+k_{1}$ and $\partial_{\mu} h_{r}=0$, we can also get results similar to (207) and (213).

Therefore, we cannot find the solution which exhibits an accelerating expansion of our Universe.

The power exponents of the scale factor of possible four-dimensional cosmological models are given by $a(\tilde{\mathrm{M}}) \propto \tau^{\lambda(\tilde{\mathrm{M}})}$, where $\tau$ is the cosmic time, and $a(\tilde{\mathrm{M}})$ and $a_{\mathrm{E}}(\tilde{\mathrm{M}})$ denote the scale factors of the space $\tilde{\mathrm{M}}$ in Jordan and Einstein frames with the exponents carrying the same suffices, respectively. Here $\tilde{M}$ denotes the spatial part of the spacetime M.

Since the time dependence in the metric comes from only one brane in the intersections, the obtained expansion law is simple. In order to find an expanding universe, one may have to compactify the vacuum bulk space as well as the brane world volume. Unfortunately we find that the fastest expanding case in the Jordan frame has the power $\lambda(\tilde{\mathrm{M}})<1 / 2$, which is too small to give a realistic expansion law like that in the matter-dominated era $\left(a \propto \tau^{2 / 3}\right)$ or that in the radiation-dominated era $\left(a \propto \tau^{1 / 2}\right)$.

When we compactify the extra dimensions and go to the four-dimensional Einstein frame, the power exponents are differently depending on how we compactify the extra dimensions even within one solution. For M-brane in the 11-dimensional theory, we give the power exponent of the fastest expansion of our four-dimensional Universe in the Einstein frame. We again see that the expansion is too small. Hence, we have to conclude that in order to find a realistic expansion of the Universe in this type of models, one has to include additional "matter" fields on the brane.

These are the same results as the case of the delocalized intersecting brane solutions. For the solutions (213) involving two intersecting brane in the 10- or 11-dimensional theories which are related to the supergravity, we can see the power exponents of the scale factor of possible fourdimensional FLRW cosmological models in the Tables in [4].

\section{B. The intersection of brane and KK-monopole system}

Next we apply the dynamical intersecting brane solutions including KK-monopoles. We should look for whether there is a solution with an isotropic and homogeneous three space. The $D$-dimensional metric (40) can be expressed as

$$
\begin{aligned}
d s^{2}= & -h^{a}(t, y, z) d t^{2}+d s^{2}(\tilde{\mathrm{X}})+d s^{2}(\mathrm{Y})+d s^{2}(\mathrm{Z}) \\
& +h^{b}(t, y, z) h_{k}^{-1}(z)\left(d v+A_{a} d z^{a}\right)^{2},
\end{aligned}
$$

where we have defined

$$
\begin{aligned}
d s^{2}(\tilde{\mathrm{X}}) & \equiv h^{a}(t, y, z) \delta_{P Q}(\tilde{\mathrm{X}}) d \theta^{P} d \theta^{Q}, \\
d s^{2}(\mathrm{Y}) & \equiv h^{b}(t, y, z) \gamma_{i j}(\mathrm{Y}) d y^{i} d y^{j}, \\
d s^{2}(\mathrm{Z}) & \equiv h^{b}(t, y, z) h_{k}(z) u_{a b}(\mathrm{Z}) d z^{a} d z^{b} .
\end{aligned}
$$

Here, $a, b$ are given by (41), and $\delta_{P Q}(\tilde{\mathrm{X}})$ is the $p$-dimensional Euclidean metric, and $\theta^{P}$ denotes the coordinate of the $p$-dimensional Euclid space $\tilde{\mathrm{X}}$.

In the following, we set $h=A t+h_{1}(y, z)$. The $D$-dimensional metric (215) can be written as

$$
\begin{aligned}
d s^{2}= & {\left[1+\left(\frac{\tau}{\tau_{0}}\right)^{-2 /(a+2)} h_{1}\right]^{a}\left[-d \tau^{2}+\left(\frac{\tau}{\tau_{0}}\right)^{2 a /(a+2)} \delta_{P Q}(\tilde{\mathrm{X}}) d \theta^{P} d \theta^{Q}\right.} \\
& \left.+\left\{1+\left(\frac{\tau}{\tau_{0}}\right)^{-2 /(a+2)} h_{1}\right\}\left(\frac{\tau}{\tau_{0}}\right)^{2 b /(a+2)}\left\{\gamma_{i j}(\mathrm{Y}) d y^{i} d y^{j}+h_{k} u_{a b}(\mathrm{Z}) d z^{a} d z^{b}+h_{k}^{-1}\left(d v+A_{a} d z^{a}\right)^{2}\right\}\right],
\end{aligned}
$$




\section{MASATO MINAMITSUJI AND KUNIHITO UZAWA}

where we have introduced the cosmic time $\tau$ defined by

$$
\frac{\tau}{\tau_{0}}=(A t)^{(a+2) / 2}, \quad \tau_{0}=\frac{2}{(a+2) A} .
$$

Now we again apply these solutions to lowerdimensional effective theory. We compactify $d(\equiv$ $d_{1}+d_{2}+d_{3}$ ) dimensions to fit our Universe, where $d_{1}$, $d_{2}$, and $d_{3}$ denote the compactified dimensions with respect to the $\tilde{X}, Y$, and $\mathrm{Z}$ spaces. The metric (215) is then described by

$$
d s^{2}=d s^{2}(\mathrm{M})+d s^{2}(\mathrm{~N})
$$

where $d s^{2}(\mathrm{M})$ is the $(D-d)$-dimensional metric and $d s^{2}(\mathrm{~N})$ is the metric of compactified dimensions.

By the conformal transformation

$$
d s^{2}(\mathrm{M})=h^{B} h_{k}^{C} d s^{2}(\overline{\mathrm{M}}),
$$

PHYSICAL REVIEW D 84, 126006 (2011)

we can rewrite the $(D-d)$-dimensional metric in the Einstein frame. Here $B$ and $C$ are

$$
B=-\frac{a d_{1}+b\left(d_{2}+d_{3}\right)}{D-d-2}, \quad C=-\frac{2 d_{3}}{D-d-2} .
$$

Hence, the $(D-d)$-dimensional metric in the Einstein frame is

$$
\begin{aligned}
d s^{2}(\overline{\mathrm{M}})= & h^{B^{\prime}} h_{k}^{-C}\left[-d t^{2}+\delta_{P^{\prime} Q^{\prime}}\left(\tilde{\mathrm{X}}^{\prime}\right) d \theta^{P^{\prime}} d \theta^{Q^{\prime}}\right. \\
& +h^{4 / N}\left\{\gamma_{k^{\prime} l^{\prime}}\left(\mathrm{Y}^{\prime}\right) d y^{k^{\prime}} d y^{l^{\prime}}+h_{k} u_{a^{\prime} b^{\prime}}\left(\mathrm{Z}^{\prime}\right) d z^{a^{\prime}} d z^{b^{\prime}}\right. \\
& \left.\left.+h_{k}^{-1}\left(d v+A_{a^{\prime}} d z^{a^{\prime}}\right)^{2}\right\}\right],
\end{aligned}
$$

where $B^{\prime}$ is defined by $B^{\prime}=-B+a$, and $\tilde{X}^{\prime}, \mathrm{Y}^{\prime}$, and $\mathrm{Z}^{\prime}$ denote the $\left(p-d_{1}\right)-,\left(D-5-p-d_{2}\right)-$, and $\left(3-d_{3}\right)$-dimensional spaces, respectively.

For $h=A t+h_{1}(y, z)$, the metric (222) is thus rewritten as

$$
\begin{aligned}
d s^{2}(\overline{\mathrm{M}})= & h_{s}^{C^{\prime}}\left[1+\left(\frac{\tau}{\tau_{0}}\right)^{-2 /\left(B^{\prime}+2\right)} h_{1}\right]^{B^{\prime}}\left[-d \tau^{2}+\left(\frac{\tau}{\tau_{0}}\right)^{2 B^{\prime} /\left(B^{\prime}+2\right)} \delta_{P^{\prime} Q^{\prime}}\left(\tilde{\mathrm{X}}^{\prime}\right) d \theta^{P^{\prime}} d \theta^{Q^{\prime}}+\left\{1+\left(\frac{\tau}{\tau_{0}}\right)^{-2 /\left(B^{\prime}+2\right)} h_{1}\right\}\right. \\
& \left.\times\left(\frac{\tau}{\tau_{0}}\right)^{2\left(B^{\prime}+1\right) /\left(B^{\prime}+2\right)}\left\{\gamma_{k^{\prime} l^{\prime}}\left(\mathrm{Y}^{\prime}\right) d y^{k^{\prime}} d y^{l^{\prime}}+h_{k} u_{a^{\prime} b^{\prime}}\left(\mathrm{Z}^{\prime}\right) d z^{a^{\prime}} d z^{b^{\prime}}+h_{k}^{-1}\left(d v+A_{a^{\prime}} d z^{a^{\prime}}\right)^{2}\right\}\right]
\end{aligned}
$$

where the cosmic time $\tau$ is defined by

$$
\frac{\tau}{\tau_{0}}=(A t)^{\left(B^{\prime}+2\right) / 2}, \quad \tau_{0}=\frac{2}{\left(B^{\prime}+2\right) A} .
$$

\begin{tabular}{|c|c|c|c|c|c|c|c|c|c|c|c|c|c|c|}
\hline Branes & & 0 & 1 & 2 & 3 & 4 & 5 & 6 & 7 & 8 & 9 & $\tilde{M}$ & $\lambda(\tilde{\mathrm{M}})$ & $\lambda_{\mathrm{E}}(\tilde{\mathrm{M}})$ \\
\hline & D0 & $\circ$ & & & & & & & & & & & $\lambda(\mathrm{Y})=1 / 9$ & $\lambda_{\mathrm{E}}\left(\mathrm{Y}_{1}\right)=\frac{1}{9-d_{2}-d_{3}}$ \\
\hline \multirow[t]{3}{*}{ D0-KK } & KK & $\circ$ & $\circ$ & $\circ$ & $\circ$ & $\circ$ & $\circ$ & & $A_{1}$ & $A_{2}$ & $A_{3}$ & $\mathrm{Y} \& v \& \mathrm{Z}$ & $\lambda(v)=1 / 9$ & $\lambda_{\mathrm{E}}(v)=\frac{1}{9-d_{2}-d_{3}}$ \\
\hline & $x^{N}$ & $t$ & $y^{1}$ & $y^{2}$ & $y^{3}$ & $y^{4}$ & $y^{5}$ & $v$ & $z^{1}$ & $z^{2}$ & $z^{3}$ & & $\lambda(Z)=1 / 9$ & $\lambda_{\mathrm{E}}(\mathrm{Z})=\frac{1}{9-d_{2}-d_{3}}$ \\
\hline & D1 & $\circ$ & $\circ$ & & & & & & & & & & $\lambda(\mathrm{Y})=1 / 5$ & $\lambda_{\mathrm{E}}(\mathrm{Y})=\frac{2-d_{1}}{10-2 d_{1}-d_{2}-d_{3}} \ell$ \\
\hline \multirow[t]{3}{*}{ D1-KK } & KK & $\circ$ & $\circ$ & $\circ$ & $\circ$ & $\circ$ & $\circ$ & & $A_{1}$ & $A_{2}$ & $A_{3}$ & $\mathrm{Y} \& v \& \mathrm{Z}$ & $\lambda(v)=1 / 5$ & $\lambda_{\mathrm{E}}(v)=\frac{2-d_{1}}{10-2 d_{1}-d_{2}-d_{3}}$ \\
\hline & $x^{N}$ & $t$ & $x$ & $y^{1}$ & $y^{2}$ & $y^{3}$ & $y^{4}$ & $v$ & $z^{1}$ & $z^{2}$ & $z^{3}$ & & $\lambda(Z)=1 / 5$ & $\lambda_{\mathrm{E}}(\mathrm{Z})=\frac{2-d_{1}}{10-2 d_{1}-d_{2}-d_{3}} \nabla$ \\
\hline & D2 & $\circ$ & $\circ$ & $\circ$ & & & & & & & & & $\lambda(\mathrm{Y})=3 / 11$ & $\lambda_{\mathrm{E}}(\mathrm{Y})=\frac{3-d_{1}}{11-2 d_{1}-d_{2}-d_{3}}$ \\
\hline \multirow[t]{3}{*}{ D2-KK } & KK & $\circ$ & $\circ$ & $\circ$ & $\circ$ & $\circ$ & $\circ$ & & $A_{1}$ & $A_{2}$ & $A_{3}$ & $\mathrm{Y} \& v \& \mathrm{Z}$ & $\lambda(v)=3 / 11$ & $\lambda_{\mathrm{E}}(v)=\frac{3-d_{1}}{11-2 d_{1}-d_{2}-d_{3}}$ \\
\hline & $x^{N}$ & $t$ & $x^{1}$ & $x^{2}$ & $y^{1}$ & $y^{2}$ & $y^{3}$ & $v$ & $z^{1}$ & $z^{2}$ & $z^{3}$ & & $\lambda(\mathrm{Z})=3 / 11$ & $\lambda_{\mathrm{E}}(\mathrm{Z})=\frac{3-d_{1}}{11-2 d_{1}-d_{2}-d_{3}}$ \\
\hline & D3 & $\circ$ & $\circ$ & $\circ$ & $\circ$ & & & & & & & $\tilde{\mathrm{X}}$ & $\lambda(\tilde{X})=-1 / 3$ & $\lambda_{\mathrm{E}}(\tilde{\mathrm{X}})=\frac{d_{2}+d_{3}+d_{4}-4}{12-2 d_{1}-d_{2}-d_{3}}$ \\
\hline \multirow[t]{3}{*}{ D3-KK } & KK & $\circ$ & $\circ$ & $\circ$ & $\circ$ & $\circ$ & $\circ$ & & $A_{1}$ & $A_{2}$ & $A_{3}$ & $\mathrm{Y} \& v \& \mathrm{Z}$ & $\lambda(\mathrm{Y})=1 / 3$ & $\lambda_{\mathrm{E}}(\mathrm{Y})=\frac{4-d_{1}}{12-2 d_{1}-d_{2}-d_{3}} /$ \\
\hline & $x^{N}$ & $t$ & $x^{1}$ & $x^{2}$ & $x^{3}$ & $y^{1}$ & $y^{2}$ & $v$ & $z^{1}$ & $z^{2}$ & $z^{3}$ & & $\lambda(v)=\lambda(\mathrm{Z})=1 / 3$ & $\lambda_{\mathrm{E}}(v)=\lambda_{\mathrm{E}}(\mathrm{Z})=\frac{4-d_{1}}{12-2 d_{1}-d_{2}-d_{3}} \mu \mathrm{m}$ \\
\hline & D4 & $\circ$ & $\circ$ & $\circ$ & $\circ$ & $\circ$ & & & & & & & $\lambda(\tilde{\mathrm{X}})=-3 / 13$ & $\lambda_{\mathrm{E}}(\tilde{\mathrm{X}})=\frac{d_{2}+d_{3}+d_{4}-3}{13-2 d_{1}-d_{2}-d_{3}}$ \\
\hline \multirow[t]{2}{*}{ D4-KK } & KK & $\circ$ & $\circ$ & $\circ$ & $\circ$ & $\circ$ & $\circ$ & & $A_{1}$ & $A_{2}$ & $A_{3}$ & $\mathrm{Y} \& v \& \mathrm{Z}$ & $\lambda(\mathrm{Y})=3 / 11$ & $\lambda_{\mathrm{E}}(\mathrm{Y})=\frac{5-d_{1}}{13-2 d_{1}-d_{2}-d_{3}}$ \\
\hline & $x^{N}$ & $t$ & $x^{1}$ & $x^{2}$ & $x^{3}$ & $x^{4}$ & $y$ & $v$ & $z^{1}$ & $z^{2}$ & $z^{3}$ & & $\lambda(v)=\lambda(Z)=5 / 13$ & $\lambda_{\mathrm{E}}(v)=\lambda_{\mathrm{E}}(\mathrm{Z})=\frac{5-d_{1}}{13-2 d_{1}-d_{2}-d_{3}}$ \\
\hline
\end{tabular}

The $(D-d)$-dimensional metric (223) shows that there is no solution which exhibits an accelerating expansion of our Universe.

We list the FLRW cosmological solutions with an isotropic and homogeneous three-space for the solutions (223) in Table IV for M-branes, Table V for D-branes, and Table VI

TABLE V. Pair intersections between $\mathrm{D} p(p \leq 4)$-brane and KK-monopole in $D=10$ with dependence on overall transverse coordinates. 
TABLE VI. Pair intersections between fundamental string and KK-monopole in $D=10$ with dependence on overall transverse coordinates.

\begin{tabular}{lccccccccccccccc}
\hline \hline Branes & & 0 & 1 & 2 & 3 & 4 & 5 & 6 & 7 & 8 & 9 & & $\tilde{\mathrm{M}}$ & $\lambda(\tilde{\mathrm{M}})$ & $\lambda_{\mathrm{E}}(\tilde{\mathrm{M}})$ \\
\hline & $\mathrm{F} 1$ & $\circ$ & $\circ$ & & & & & & & & & $\sqrt{ }$ & & $\lambda(\mathrm{Y})=1 / 5$ & $\lambda_{\mathrm{E}}(\mathrm{Y})=\frac{2-d_{1}}{10-2 d_{1}-d_{3}}$ \\
F1-KK & $\mathrm{KK}$ & $\circ$ & $\circ$ & $\circ$ & $\circ$ & $\circ$ & $\circ$ & & $A_{1}$ & $A_{2}$ & $A_{3}$ & & $\mathrm{Y} \& v \& \mathrm{Z}$ & $\lambda(v)=1 / 5$ & $\lambda_{\mathrm{E}}(v)=\frac{2-d_{1}-d_{3}}{10-2 d_{1}-d_{2}-d_{3}}$ \\
& $x^{N}$ & $t$ & $x$ & $y^{1}$ & $y^{2}$ & $y^{3}$ & $y^{4}$ & $v$ & $z^{1}$ & $z^{2}$ & $z^{3}$ & & & $\lambda(\mathrm{Z})=1 / 5$ & $\lambda_{\mathrm{E}}(\mathrm{Z})=\frac{2-d_{1}}{10-2 d_{1}-d_{2}-d_{3}}$ \\
\hline \hline
\end{tabular}

for F1 and NS5-branes. We find the power exponents of the scale factor of possible four-dimensional cosmological models as $a(\tilde{\mathrm{M}}) \propto \tau^{\lambda(\tilde{\mathrm{M}})}$, where $a(\tilde{\mathrm{M}})$ and $a_{\mathrm{E}}(\tilde{\mathrm{M}})$ denote the scale factors of our Universe $\tilde{M}$ in Jordan and Einstein frames with the exponents carrying the same suffices, respectively. Here $\tilde{M}$ denotes the spatial part of the spacetime M.

In the KK-monopole solution, the expansion law is easily obtained because the time dependence in the metric comes from only $p$-brane in the intersections. We can find an expanding universe even if one may compactify the vacuum bulk space as well as the brane world volume. However, it is impossible to obtain the cosmological model whose scale factor has the power $\lambda(\tilde{\mathrm{M}})>1 / 2$ in the Jordan frame. Then they cannot give a realistic expansion law like that in the matter-dominated era $\left(a \propto \tau^{2 / 3}\right)$ or that in the radiation-dominated era $\left(a \propto \tau^{1 / 2}\right)$.

The power exponents in the four-dimensional Einstein frame after compactifing the extra dimensions are different depending on how we compactify the extra dimensions even within one solution. For M-brane and D-brane in the

TABLE VII. The power exponent of the fastest expansion in the Einstein frame for M-brane, D-brane, fundamental string in KK-monopole background. "TD" in the table shows which brane is time dependent.

\begin{tabular}{|c|c|c|c|c|c|}
\hline Branes & TD & $\operatorname{dim}(M)$ & $\overline{\mathrm{M}}$ & $\left(d_{1}, d_{2}, d_{3}\right)$ & $\lambda_{\mathrm{E}}(\overline{\mathrm{M}})$ \\
\hline \multirow[t]{2}{*}{ M2-KK } & M2 & 8 & $\mathrm{Y} \& v \& \mathrm{Z}$ & $(0,1,2)$ & $1 / 3$ \\
\hline & M2 & 8 & Y \& Z & $(0,3,0)$ & $1 / 3$ \\
\hline \multirow[t]{2}{*}{ M5-KK } & M5 & 7 & $\tilde{\mathrm{X}} \& \mathrm{Y} \& v \& \mathrm{Z}$ & $(2,0,2)$ & $-1 / 9$ \\
\hline & M5 & 9 & $\tilde{\mathrm{X}} \& \mathrm{Y} \& v \& \mathrm{Z}$ & $(0,0,2)$ & $6 / 13$ \\
\hline \multirow[t]{2}{*}{ D0-KK } & D0 & 6 & $\mathrm{Y} \& v \& \mathrm{Z}$ & $(0,2,2)$ & $1 / 5$ \\
\hline & D0 & 5 & Y\& $v \& Z$ & $(0,3,2)$ & $1 / 4$ \\
\hline \multirow[t]{2}{*}{ D1-KK } & D1 & 7 & $\mathrm{Y} \& v \& \mathrm{Z}$ & $(0,1,2)$ & $2 / 7$ \\
\hline & D1 & 5 & $\mathrm{Y} \& v \& \mathrm{Z}$ & $(0,3,2)$ & $2 / 5$ \\
\hline \multirow[t]{2}{*}{ D2-KK } & D2 & 8 & $\mathrm{Y} \& v \& \mathrm{Z}$ & $(0,0,2)$ & $1 / 3$ \\
\hline & D2 & 6 & $\mathrm{Y} \& v \& \mathrm{Z}$ & $(0,2,2)$ & $3 / 7$ \\
\hline \multirow[t]{2}{*}{ D3-KK } & D3 & 7 & $\tilde{\mathrm{X}} \& \mathrm{Y} \& v \& \mathrm{Z}$ & $(0,1,2)$ & $-1 / 9$ \\
\hline & D3 & 7 & $\tilde{\mathrm{X}} \& \mathrm{Y} \& v \& \mathrm{Z}$ & $(0,1,2)$ & $4 / 9$ \\
\hline \multirow[t]{2}{*}{ D4-KK } & D4 & 8 & $\tilde{\mathrm{X}} \& \mathrm{Y} \& v \& \mathrm{Z}$ & $(0,0,2)$ & $-1 / 11$ \\
\hline & D4 & 8 & $\tilde{\mathrm{X}} \& \mathrm{Y} \& v \& \mathrm{Z}$ & $(0,0,2)$ & $5 / 11$ \\
\hline \multirow[t]{2}{*}{ F1-KK } & $\mathrm{F} 1$ & 7 & $\mathrm{Y} \& v \& \mathrm{Z}$ & $(0,1,2)$ & $2 / 7$ \\
\hline & F1 & 5 & $\mathrm{Y} \& v \& \mathrm{Z}$ & $(0,3,2)$ & $2 / 5$ \\
\hline
\end{tabular}

10- or 11-dimensional theory, we give the power exponent of the fastest expansion of our four-dimensional Universe in the Einstein frame in Table VII. However, the expansion is too small to find a realistic expansion of the Universe in the KK-monopole background. Then it is necessary to add some corrections in the background to obtain a realistic cosmological solution.

\section{DISCUSSIONS}

In this paper, we have studied dynamical solutions of $p$-brane. In the case of partially localized static intersecting brane solutions, even the metric ansatz in terms of harmonic functions which would generalize such restricted metric ansatz for the delocalized brane case is known, we could mention the explicit expressions for harmonic functions. We have applied simple coordinate transformations to the differential equations satisfied by the harmonic functions in order to bring them to the forms of partial differential equations which have known explicit solutions. The Einstein equations give the intersection rules which the dynamical brane have to obey. Because of the simplicity of the intersection rule, it is easy to work out obtaining the explicit analytical form of the solution for the field equations in the $D$-dimensional projective. The intersection rules have led to the results that harmonic functions can be written by linear combinations of terms depending on both coordinates of world volume and transverse space. Moreover, in the case of 10- or 11-dimensional theories, the form of the harmonic function implies that the dynamical solution becomes the supersymmetric one if we drop the time dependence. We also find that the field strengths vanish if we take a limit where the coordinate dependence with respect to transverse space becomes much smaller. This turned out to be vacuum solutions if the scalar field is trivial because in this limit the scalar and gauge fields do not contribute the energy momentum tensor, which presumably does not affect the model. We can understand this as a Kasner-type metric. This feature is expected to be seen in a wide class of supersymmetric solutions beyond the examples considered in the present paper.

The dynamical solutions include the dilaton coupling parameter $N$ in which appears the exact forms of the field strengths. We observed that obtaining the explicit analytical form of dynamical solutions is almost impossible with $N \neq 4$, since harmonic functions that specify branes now 
satisfy coupled partial differential equations. If we set $N=4$, these are apparently related to the classical solutions of string theory and certainly have a lot of attractive properties. Firstly, these solutions were obtained by replacing the time-independent warp factor of the static solution with the time-dependent function. Secondly, our solutions can contain only one function depending on both time as well as overall or relative transverse space coordinates because the Einstein equations tell us that either (i) two branes depend only on the coordinates along the relative and overall transverse directions or (ii) while one brane is completely dynamical the other brane has to depend only on the coordinates along the relative and overall transverse directions. A new class of solutions where all harmonic functions depend on time is more challenging.

We have shown that each solution gives a FLRW universe if we regard the homogeneous and isotropic part of the brane world-volume or transverse space as our spacetime. However, the power of the scale factor is so small that the solutions of field equations cannot give a realistic expansion law. This means that we have to consider additional matter on the brane in order to get a realistic expanding universe. The solutions implies that as the number $p$ increases, the power of the scale factor becomes large. We have found that the intersection with D6-brane in ten-dimensional theory gives the fastest expansion of our Universe because the three-dimensional spatial space of our Universe stays in the transverse space to the D6-brane. Though the power of the scale factor for the transverse space in solutions with the D7- or D8-branes is larger than those with the D6-brane, the number of the transverse space to these branes is less than three. Hence, these solutions cannot provide the isotropic universe if we assume that the transverse space to the brane is the part of our Universe. These are same results as the case of delocalized brane solutions [4,5].

The method described here can of course be applied in this model to all other intersecting brane systems involving more than three branes. The same equations, given by (18c) will lead to the coordinate dependence of the metric because of the ansatz for the fields. Hence, only one of branes will appear exhibit time dependence, as we have already discussed in Secs. III and IV through II. A serious problem is the difficulty of constructing realistic cosmological models such as inflationary universe scenario of the early universe or the accelerating expansion of the present universe. This problem is of course avoided in delocalized brane solutions with particular coupling between scalar field and gauge field strength, which does not apparently relate to the classical solutions of string theory.
Then, beyond the model discussed here, obtaining the accelerating expansion of our Universe in a string theory, there is more realistic problem of setting the field ansatz, coupling constant and internal space in a theory of coupled scalar, gauge and gravitational fields. This is more complicated, because even in the case of using the same field ansatz as the supersymmetric solutions there are coupled partial differential equations which in general do not have simple form. Hence, that alone should not prevent the method described here from being applicable to realistic theories, at least for $\mathrm{D} p_{r}-\mathrm{D} p_{s}$ branes, since a lot of terms in the field equations cannot be eliminated by including enough fields. If the coupling constant that involve fields have a single parameter $N$ attached to matter fields, then we can introduce a parameter for the fields by coupling scalar field with the harmonic functions to the gauge field strengths. But it is not clear how to deal with the relation between the string theory and containing parameters to which are attached two or more field strengths. This raises the question whether the dynamical brane solution is really related to the supersymmetric solutions because the value of the coupling constant in these solutions are in general severely restricted. It is difficult to obtain the de Sitter compactification model which is consistent with the string theory [29-31]. There is something mysterious about this. The actual calculations in this paper were done for a fixed field ansatz. Dynamical delocalized brane solutions would have been done in the same way, which had never give an accelerating expansion of universe in the string theory.

The lower-dimensional effective theory for the intersection of two branes and branes on KK-monopole or wave could almost have been discussed with same calculation as in the case of dynamical delocalized branes. The moduli potential in the lower-dimensional effective theories after compactifications gives the flat direction. Hence, the solutions we have obtained may give some moduli instabilities. It would be necessary to introduce some corrections in the effective theory to fix the volume and shape moduli of the internal space. Otherwise, the moduli instabilities will grow unless the global or local minimum of the potential. Such an effective theory was briefly mentioned in [4], and proposed and developed in some detail by $[2,24]$.

\section{ACKNOWLEDGMENTS}

K. U. would like to thank H. Kodama, M. Sasaki, N. Ohta and T. Okamura for continuing encouragement. K. U. is supported by Grant-in-Aid for Young Scientists (B) of JSPS Research, under Contract No. 20740147. 
[1] P. Binetruy, M. Sasaki, and K. Uzawa, Phys. Rev. D 80, 026001 (2009).

[2] K. i. Maeda, N. Ohta, and K. Uzawa, J. High Energy Phys. 06 (2009) 051.

[3] G. W. Gibbons and K. i. Maeda, Phys. Rev. Lett. 104, 131101 (2010).

[4] M. Minamitsuji, N. Ohta, and K. Uzawa, Phys. Rev. D 82, 086002 (2010).

[5] M. Minamitsuji and K. Uzawa, Phys. Rev. D 83, 086002 (2011).

[6] G. W. Gibbons, H. Lu, and C. N. Pope, Phys. Rev. Lett. 94, 131602 (2005).

[7] K. i. Maeda, N. Ohta, M. Tanabe, and R. Wakebe, J. High Energy Phys. 06, 036 (2009).

[8] K. i. Maeda and M. Nozawa, Phys. Rev. D 81, 044017 (2010).

[9] K. i. Maeda, N. Ohta, M. Tanabe, and R. Wakebe, J. High Energy Phys. 04 (2010) 013.

[10] M. Minamitsuji, N. Ohta, and K. Uzawa, Phys. Rev. D 81, 126005 (2010).

[11] K. i. Maeda and M. Nozawa, Phys. Rev. D 81, 124038 (2010).

[12] M. Nozawa and K. i. Maeda, Phys. Rev. D 83, 024018 (2011).

[13] K. i. Maeda and M. Nozawa, arXiv:1104.1849.

[14] H. s. Yang, arXiv:hep-th/9902128.

[15] A. A. Tseytlin, Mod. Phys. Lett. A 11, 689 (1996).
[16] A.A. Tseytlin, Classical Quantum Gravity 14, 2085 (1997).

[17] D. Youm, Phys. Rept. 316, 1 (1999).

[18] D. Youm, Nucl. Phys. B 556, 222 (1999).

[19] A. A. Tseytlin, Nucl. Phys. B 475, 149 (1996).

[20] R. Argurio, F. Englert, and L. Houart, Phys. Lett. B 398, 61 (1997).

[21] R. Argurio, arXiv:hep-th/9807171.

[22] N. Ohta, Phys. Lett. B 403, 218 (1997).

[23] H. Kodama and K. Uzawa, J. High Energy Phys. 07 (2005) 061.

[24] H. Kodama and K. Uzawa, J. High Energy Phys. 03 (2006) 053.

[25] J. M. Figueroa-O'Farrill, Classical Quantum Gravity 17, 2925 (2000).

[26] J. M. Figueroa-O'Farrill and J. Simon, Classical Quantum Gravity 19, 6147 (2002).

[27] E. Bergshoeff, M. de Roo, E. Eyras, B. Janssen, and J. P. vander Schaar, Classical Quantum Gravity 14, 2757 (1997).

[28] E. Bergshoeff, M. de Roo, E. Eyras, B. Janssen, and J. P. vander Schaar, Nucl. Phys. B 494, 119 (1997).

[29] K. i. Maeda, M. Minamitsuji, N. Ohta, and K. Uzawa, Phys. Rev. D 82, 046007 (2010).

[30] M. Minamitsuji and K. Uzawa, arXiv:1103.5325.

[31] M. Minamitsuji and K. Uzawa, arXiv:1103.5326. 